https://doi.org/10.15407/ujpe64.10.888

O.V. PROKOPENKO,${ }^{1}$ D.A. BOZHKO,${ }^{2,3}$ V.S. TYBERKEVYCH,${ }^{4}$ A.V. CHUMAK,${ }^{5,6}$

V.I. VASYUCHKA, ${ }^{6}$ A.A. SERGA,${ }^{6}$ O. DZYAPKO,${ }^{7}$ R.V. VERBA, ${ }^{8}$

A.V. TALALAEVSKIJ,${ }^{9}$ D.V. SLOBODIANIUK, ${ }^{1}$ YU.V. KOBLJANSKYJ,${ }^{1}$

V.A. MOISEIENKO ${ }^{1}$ S.V. SHOLOM,${ }^{10}$ V.YU. MALYSHEV ${ }^{1}$

1 Taras Shevchenko National University of Kyiv,

Faculty of Radiophysics, Electronics, and Computer Systems

(Kyiv 01601, Ukraine; e-mail: oleksandr.prokopenko@gmail.com)

2 James Watt School of Engineering, University of Glasgow

(Glasgow G12 8LT, United Kingdom)

3 Department of Physics and Energy Science, University of Colorado at Colorado Springs

(Colorado Springs CO 80918,USA; e-mail: dbozhko@uccs.edu)

${ }^{4}$ Department of Physics, Oakland University

(Rochester MI 48309, USA; e-mail: tyberkev@oakland.edu)

${ }^{5}$ Faculty of Physics, University of Vienna

(A-1090Wien, Austria; e-mail: andrii.chumak@univie.ac.at)

${ }^{6}$ Fachbereich Physik and Landesforschungszentrum OPTIMAS,

Technische Universität Kaiserslautern

(67663 Kaiserslautern, Germany; e-mail: vasyucka@physik.uni-kl.de,serga@physik.uni-kl.de)

${ }^{7}$ Institute for Applied Physics and Center for Nonlinear Science, University of Muenster

(48149 Münster, Germany; e-mail: odzyapko@gmail.com)

8 Institute of Magnetism

(Kyiv 03680, Ukraine; e-mail: verrv@ukr.net)

${ }^{9}$ Department of Physics, University of Regensburg

(Regensburg 93053, Germany; e-mail: aleksandrtaloon@gmail.com)

${ }^{10}$ Department of Physics, Oklahoma State University

(Stillwater, OK 740\%4,USA; e-mail: sergey.sholom@okstate.edu)

\title{
RECENT TRENDS IN MICROWAVE MAGNETISM AND SUPERCONDUCTIVITY
}

We review the development trends in microwave magnetism and superconductivity over the last five decades. The review contains the key results of recent studies related to the promising areas of modern magnetism and applied physics - spintronics, magnonics, magnon caloritronics, physics of magnonic crystals, spin-wave logic, and the development of novel micro- and nanoscale magnetic devices. The main achievements in these fields of physics are summarized and generalized.

Ke ywords: spin wave, magnonic crystal, spin-wave logic, spin-wave soliton, spin-wave bullet, spin-wave droplet, parametric pumping, magnon gas, kinetic instability, Bose-Einstein condensate, magnon superfluidity, high-temperature superconductivity, Josephson junction, microwave devices.

(C) O.V. PROKOPENKO, D.A. BOZHKO, V.S. TYBERKEVYCH, A.V. CHUMAK, V.I. VASYUCHKA, A.A. SERGA, O. DZYAPKO, R.V. VERBA, A.V. TALALAEVSKIJ, D.V. SLOBODIANIUK, YU.V. KOBLJANSKYJ, V.A. MOISEIENKO, S.V. SHOLOM, V.YU. MALYSHEV, 2019

\section{Introduction}

A revision of the basic concepts of physics in the 1920s and 1930s led to the intense development of its many fields, including the solid state physics. The use of quantum mechanics made it possible to explain the phenomenon of superconductivity, discovered in 1911 by H. Kamerlingh Onnes [1]. Newly formed quantum ISSN 2071-0194. Ukr. J. Phys. 2019. Vol. 64, No. 10 
physics also helped to determine promising ways to search for new superconductors and choose the most effective methods to study them. In 1986, these studies led to the discovery of high-temperature superconductivity [2], and, in 2019, superconductivity at almost room temperatures was discovered [3].

The XX century was also the time of a rapid development of another major area of the solid state physics - the physics of magnetism. After the development of the phenomenological model of magnetization dynamics by L. Landau and E. Lifshitz in 1935 [4], and the discovery of electron paramagnetic resonance by E. Zavoisky [5] and ferromagnetic resonance by J. Griffiths [6] in 1945-1946, active studies of magnetic excitations in solids have begun. In the last decades, these studies led to the appearance of spintronics, magnonics, spin caloritronics, spinorbitronics, and other new areas of modern applied physics and nanoelectronics.

This review outlines the main trends in the development of microwave superconductivity and magnetism over the past five decades. Considering these topics, of course, we cannot but mention the significant contribution of Prof. Gennadii A. Melkov and his scientific school to these studies. His scholars became leading experts in the field of solid state physics, in particular, in magnetism and nonlinear spin-wave phenomena. Therefore, the presentation of the material in the article is based on the results obtained by G.A. Melkov and his scholars.

The review content is divided into subsections by areas, in which the most intense studies are performed and the most impact has been achieved: hightemperature superconductivity (Sec. 2, p. 889), parametric and nonlinear processes in magnets (Sec. 3, p. 891), spin-wave solitons and bullets (Sec. 4, p. 895), magnon Bose-Einstein condensates and supercurrents (Sec. 5, p. 898), magnon caloritronics (Sec. 6, p. 904), spintronics (Sec. 7, p. 906), magnonic crystals (Sec. 8, p. 908), and spin-wave logic (Sec. 9, p. 913).

\section{Physics and Applications of High-Temperature Superconductivity}

The discovery of high-temperature superconductivity phenomenon in 1986 caused a huge interest in the lowtemperature physics and techniques and in the cryogenic electronics throughout the world. G.A. Melkov, who worked at that time at the Department of Cryogenic and Microelectronics in Taras Shevchenko State
University (it was renamed later to the Department of Nanophysics and Nanoelectronics of Taras Shevchenko National University of Kyiv), also decided it is his duty to begin research on the microwave properties of high-temperature superconductors (HTS), as well as the development of microwave devices based on HTS.

Experimental and theoretical work initiated by G.A. Melkov at the department in the late 1990s developed in the following interrelated scientific areas: i) study of the microwave impedance of HTS films; ii) study of the microwave properties of superconducting surface electromagnetic wave resonators, and the creation of microwave devices on their basis; iii) development and creation of combined microwave devices with magnetic and HTS films, as well as the study of their microwave properties.

The first stage of G.A. Melkov's group activity was associated with the study of the microwave impedance of HTS films. As it is known, the microwave impedance of HTS films characterizes their quality and mainly determines the microwave properties of devices created on the basis of such films. In addition, investigating the response of an HTS film in a magnetic field to a microwave signal, one can obtain the important information necessary for a deeper understanding of the nature of high-temperature superconductivity. The first works in this scientific area were performed by G.A. Melkov with I.L. Zablotskii $[7,8]$. Further experimental researches were carried out by G.A. Melkov, A.V. Bagada, S.K. Korsak, and V.Yu. Malyshev, while theoretical models of the microwave properties of HTS films were developed jointly with A.L. Kasatkin and E.A. Pashitskii [9-13]. As a result of these studies, it has been shown that an HTS film can be considered as an inhomogeneous material consisting of series-connected areas of different types (ideal superconductor, areas with low- and large-resistance intragranular Josephson junctions, shunted by the ideal superconductor, and areas with unshunted intragranular Josephson junctions). Due to this inhomogeneity, when dc and ac magnetic fields are applied to the HTS film, it causes Abrikosov's vortices to move through the ideal superconductor areas, while the ac field causes the switching of high-resistance Josephson junction areas to their lowresistance state. In turn, this leads to the existence of unusual microwave properties of HTS films, in particular to the observation of a stronger microwave re- 
sponse of an HTS film to an ac magnetic field than the dc field response. Many years later these results also helped G.A. Melkov's colleagues and scholars to develop a novel type of Josephson junctions having improved microwave and electrical properties [14].

Later, in collaboration with Prof. V.M. Pan's group from G.V. Kurdyumov Institute for Metal Physics of the National Academy of Sciences of Ukraine (NASU) and other NASU and foreign research groups, G.A. Melkov and his colleagues showed that growth-induced linear defects strongly affect the microwave surface resistance $R_{S}$ of highly biaxially oriented HTS $\mathrm{YBa}_{2} \mathrm{Cu}_{3} \mathrm{O}_{7-\delta}$ films [15]. Such films exhibited the smallest $R_{S}(77 \mathrm{~K}, 134 \mathrm{GHz})<120 \mathrm{~m} \Omega$ and the lowest density of dislocations detected by high-resolution electron microscopy and X-ray analysis. A nature of the dislocation effect was discussed within a model of local anisotropic elastic deformation in a vicinity of dislocation cores, where the HTS critical temperature $T_{C}$ variation and an enhancement of the normal quasiparticle density are significant. Then the main features of the experimentally measured microwave surface impedance, $Z_{S}(T)$, of epitaxial $\mathrm{YBa}_{2} \mathrm{Cu}_{3} \mathrm{O}_{7-\delta}$ thin films deposited on $\mathrm{CeO}_{2}$-buffered sapphire substrates were obtained and summarized in [16, 17]: i) the surface resistance $R_{S}(T)$ at low temperatures obeys the exponential law $R_{S}(T)=R_{\text {res }}+R_{0} \exp [-\zeta / T]$ with a small gap $\zeta\left(\zeta \approx 0.7 T_{C}\right)$; ii) the most perfect quasi-singlecrystalline films reveal a distinct two-peak structure of the $R_{S}(T)$ dependence, which is not observable in films with a less ordered crystal structure. These features were believed to reveal some intrinsic electron properties of such films, namely: i) the mixed $(d+i s)$ type symmetry of the electron pairing and ii) the dominant role of extended $c$-oriented defects (e.g., edge dislocation arrays or twin planes) in the scattering of quasiparticles for the most perfect films, which demonstrates the two-peak anomalous $R_{S}(T)$ behavior.

Using the results of their research, G.A. Melkov and co-authors proposed new methods of fabrication of HTS films (Ukraine's patents \# 97010303 by January 24, 1997 and \# 24207A by October $15,2001)$. For the precise measurement of microwave properties of HTS films in the 3-cm, 8-mm, 4-mm, and $2-\mathrm{mm}$ wave bands, a unique certification center was created, which at that time had no analogs in Europe and on the territory of the former USSR.
Investigating the microwave properties of HTS films, G.A. Melkov came to the conclusion that the film itself, which has at least small, but finite, losses at microwaves, can play the role of a "two-dimensional" resonant system. Such resonators were called the surface electromagnetic wave resonators (SEWRs). Since 1997, the studies of the microwave properties of superconducting SEWRs and devices based on them had become one of the priority scientific directions in G.A. Melkov's group. In [18] G.A. Melkov and $\mathrm{Yu}$.V. Egorov developed a general theory of waves in a superconducting parallel-plate transmission line (Fig. 1, $a, b, c, d$ ). A particular case of the considered problem was the surface electromagnetic wave waveguide - an infinitely long SEWR (see Fig. 1, c). In 2000 , the initial experimental and theoretical results of these studies in X- and Ka-bands were summarized in [19] (Fig. 1, e, f).

Studies have shown that, due to the quasiuniform distribution of the electromagnetic field of a surface wave near the SEWR surface, such a resonator is a promising system for the creation of microwave devices with various integral elements, in particular Josephson junctions [18]. Using this concept, G.A. Melkov and A.M. Klushin with coauthors were able to develop and create microwave devices with up to 450 synchronized bicrystal Josephson junctions, to obtain the synchronous generation from $\sim 200$ junctions, and to create SEWRbased microwave signal detectors with Josephson junction arrays [20-24]. These studies were carried out in the $8-\mathrm{mm}$ and $3-\mathrm{cm}$ wave bands for different configurations of SEWR inside a rectangular waveguide of different cross-section sizes. In particular, it was shown that, in a below-cutoff rectangular waveguide, the spectrum of SEWR natural oscillations is substantially rarefied, which can be used for the creation of more efficient microwave devices $[25,26]$. The obtained results were summarized in [23, 24, 27].

In parallel to the study of the SEWR properties, G.A. Melkov was also involved in the creation of highquality microwave filters based on HTS films. In particular, he and his students proposed a microwave filter based on coupled SEWRs [28]. In addition, a HTS-based band-pass filter which has a frequency of 1.8 GHz and is suitable for the application in hi-end telecommunication systems was designed and their properties were investigated [29].

ISSN 2071-0194. Ukr. J. Phys. 2019. Vol. 64, No. 10 
One of the first works of G.A. Melkov in the field of applied superconductivity [30] was associated with the study of the influence of nonlinear properties of HTS films on magnetic oscillations and waves in combined structures with HTS and yttrium-iron garnet films. Later, he and his colleagues returned to this topic several times. For instance, using SEWR based on the HTS and YIG films, a high-quality X-band band-pass filter tunable by a dc magnetic field was created. Moreover, the possibility of the parametric amplification of microwave signals in HTS films was also shown [31].

In general, during almost last 30 years, G.A. Melkov with his colleagues and students made a significant contribution to the understanding of physics of high-temperature superconductors and HTS films. They also developed and created a new class of magnetically controlled superconducting microwave devices and theoretically and experimentally studied their properties.

\section{Parametric and Nonlinear Processes in Magnetic Media}

The parametric interaction in a magnetic medium arises from the fact that Landau-Lifshitz equation describing the magnetization dynamics is highly nonlinear. This leads to the coupling of different types of oscillations and waves in a magnetic system and results in their threshold excitation under certain conditions.

The most interesting effect is the excitation of magnetic oscillations and waves under the action of an external electromagnetic pumping applied to the sample. With regard for the orientation of the pumping field and the saturation magnetization, we can analyze two different cases: the so-called perpendicular and parallel pumpings.

In the case of parallel pumping process, the uniform microwave magnetic field leads to the excitation of a spin-wave pair with opposite wavevectors and the frequency equal to a half of the pumping frequency: $\omega_{k}=\omega_{-k}=\omega_{p} / 2$. One can derive equations describing the spin-wave amplitudes $c_{k}$ and $c_{-k}$ having the following form:

$$
\begin{aligned}
& \frac{d c_{k}}{d t}=i \omega_{k} c_{k}+\Gamma_{k} c_{k}+i V_{k} h_{z} e^{i \omega_{p} t} c_{-k}^{*}, \\
& \frac{d c_{-k}}{d t}=-i \omega_{k} c_{-k}+\Gamma_{-k} c_{-k}-i V_{k}^{*} h_{z} e^{-i \omega_{p} t} c_{k} .
\end{aligned}
$$

Here, $V_{k}$ is the coupling coefficient, $h_{z}$ amplitude of an external rf-field, and $\Gamma_{k}, \Gamma_{-k}$ are the dissipation
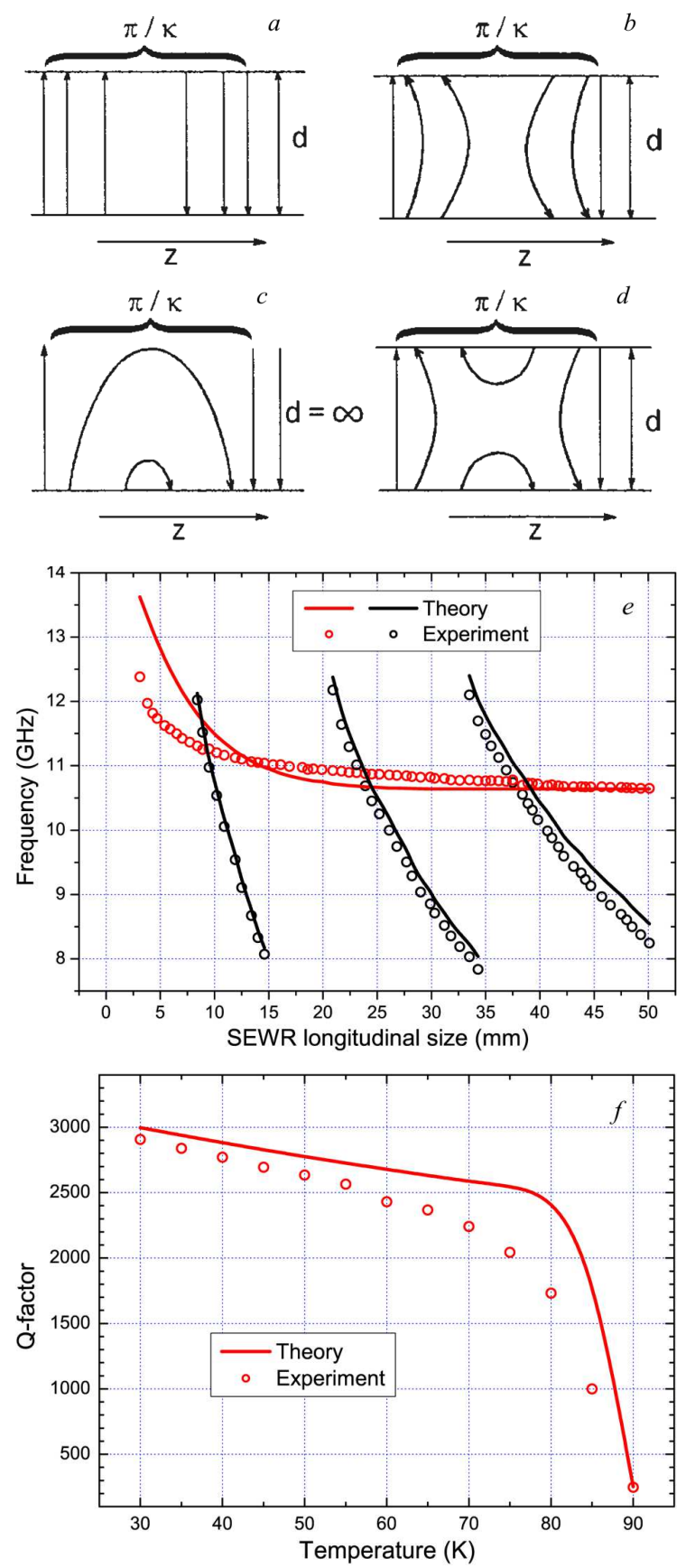

Fig. 1. Electric field lines in a parallel-plate transmission line in the cases: transverse electromagnetic wave $(a)$; Swihart wave (b); surface electromagnetic wave $(c)$; Swihart-plasmon wave (d). Microwave properties of HTS SEWR: resonance frequencies vs. the resonator longitudinal size $(e)$, red points and curves indicate the lowest surface resonance mode; temperature dependence of the resonator $Q$-factor for that mode $(f)$. Images are adopted from Refs. [18, 19] 
parameters. As one can see, the parametric interaction leads to the coupling of opposite wave-vector spin waves with the coupling coefficient being harmonic in time $\sim e^{i \omega_{p} t}$. From the general oscillation theory, it is known that this periodic modulation of the coupling can lead to the threshold excitation of coupled waves. In the simplest case where $\Gamma_{k}=\Gamma_{-k}$, the threshold condition is: $\left|h_{t h} V_{k}\right|=\Gamma_{k}$. When the amplitude of an external pumping field reach the threshold value $h_{t h}$, the amplitudes of spin waves begin to grow exponentially: $c_{k}, c_{-k} \sim e^{\left(h_{z} V_{k}-\Gamma_{k}\right) t}$. Obviously, these amplitudes cannot grow infinitely, and different nonlinearities come into play, by limiting spin-waves amplitudes. Such limiting mechanism will be discussed in what follows.

In the case of perpendicular pumping, the spinwave excitation is a two-step process. The external field excites uniform magnetization oscillations, and this leads to the coupling of spin waves with opposite wavevectors. For example, for the spin waves with frequencies close to a half of the pumping frequency, one can obtain the following equations:

$$
\begin{aligned}
& \frac{d c_{k}}{d t}=i \omega_{k} c_{k}+\Gamma_{k} c_{k}+i \rho_{k} a_{0} c_{-k}^{*}, \\
& \frac{d c_{-k}}{d t}=-i \omega_{k} c_{-k}+\Gamma_{-k} c_{-k}-i \rho_{k}^{*} a_{0}^{*} c_{k} .
\end{aligned}
$$

Here, $\rho_{k}$ is the coupling coefficient, and $a_{0}$ is the uniform mode amplitude. In this case, the excitation of a spin wave starts, when the uniform mode reaches the threshold value defined by $\left|a_{0 t h} \rho_{k}\right|=\Gamma_{k}$. This process is also called the first-order Suhl instability. In the case of second-order Suhl instability, the spin waves with frequencies $\omega_{k}=\omega_{p}$ are excited.

As was mentioned before, the spin-wave growth under the action of a parallel pumping can not last infinitely, because different nonlinear mechanisms come into play. The most important of them are: the nonlinear damping, back-influence of a spin wave on the pumping, and phase limiting mechanism. The mentioned mechanisms were investigated in [32].

The most important limiting mechanism in the case of parallel pumping is the so-called phase mechanism. The four-magnon interaction between excited spin waves leads to the appearance of a phase mismatch between the external pumping and spin waves limiting their amplitude at the level: $\left|c_{k}\right|^{2}=\frac{\sqrt{\left|h_{z} V_{p}\right|^{2}-\Gamma_{k}^{2}}}{S}$. In works $[33,34]$, G.A. Melkov and his colleagues investigated the mentioned mechanism proving its im- portant governing role in the spin-wave excitation under a parallel pumping.

The excitation of a spin-wave group with angular frequency $\omega_{k}$ can lead to further nonlinearities in a magnetic system. At high amplitudes of excited spin waves, they can become the internal pumping for other spin waves in the system leading to an additional excitation. This mechanism is called the kinetic instability and arises from the four-wave interaction in an excited magnon system. The processes $\omega_{k}+\omega_{-k}=\omega_{1}+\omega_{2}$ can be considered as a negative damping for waves with $\omega_{1}$ and $\omega_{2}$. Thus, at some critical number of excited spin waves with $\omega_{k}$, those processes can overcome the damping for spin waves with $\omega_{1}$ and lead to their excitation. Obviously, the spin waves with the lowest damping parameter are first to be excited in this way. This mechanism was discovered by G.A. Melkov and his colleagues in [35] and was investigated under various conditions in $[36-38]$.

As we can see, the parametric pumping can effectively excite spin waves with a given frequency in magnetic systems. However, the most fascinating application of parametric processes arises, when it is applied to the existing signal in a magnetic system. Let we have a spin-wave of the given frequency excited in a magnetic film by an antenna. It propagates along the film slowly loosing its amplitude due to different dissipation processes. However, if, at some point, we will apply the parametric pumping to the system, the signal spin-wave can be significantly amplified, overcoming the losses in the system. However, the most fascinating fact arises from the nature of the parametric interaction. As was shown before, this process excites a pair of waves with opposite wave vectors. This condition should be satisfied due to the momentum conservation laws in a system. In the case of already existing signal spin wave, the parametric pumping will produce an additional "idle" wave that propagates in the opposite direction. This effect is called the wave front reversal (WFR) or phase conjugation. It was first discovered in nonlinear optics and was successfully transferred to the magnetic systems by G.A. Melkov and his colleagues [39-43].

It should be noted that the optical wavefront reversal involves second-order four-wave parametric interactions pumped by counter-propagating light waves with frequencies close to those of the source and reversed signals. Spin-wave systems provided the first 


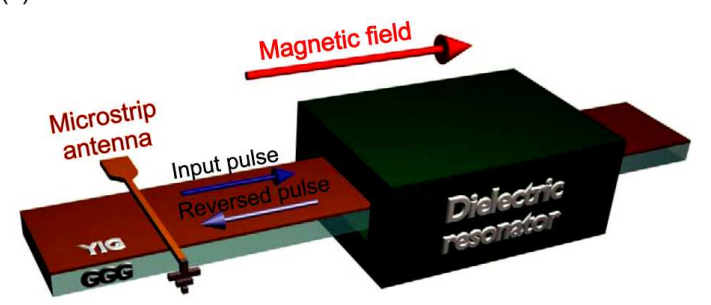

(b)
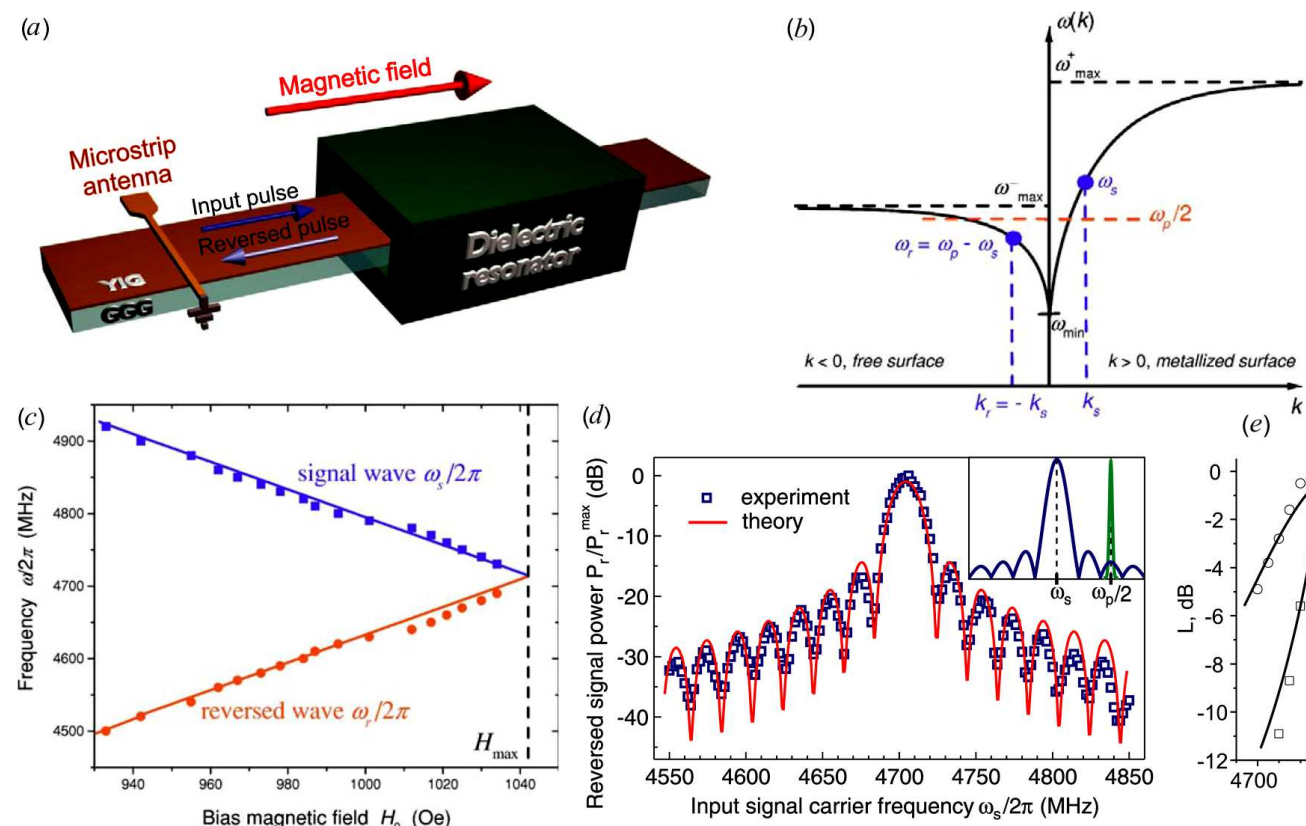

(d)

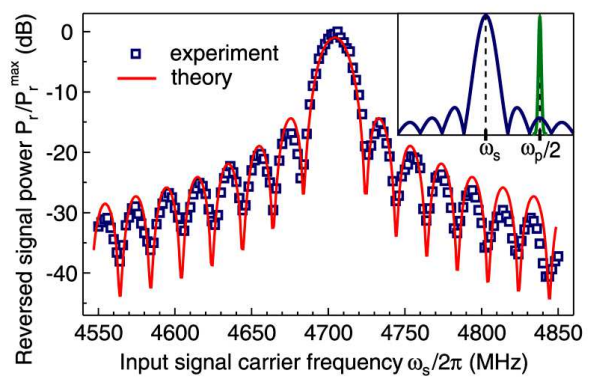

(e)

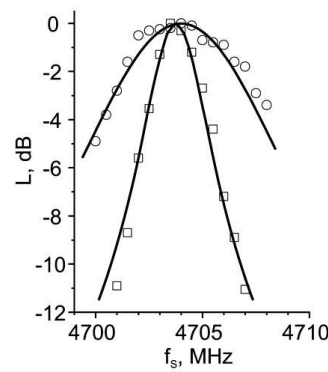

Fig. 2. Schematic experimental setup used for the nonresonant wave front reversal investigation [49] (a). Dispersion law for a one-side-metallized YIG film (surface waves) [43] (b). Dependence of the frequencies of the signal $\omega_{\mathrm{s}}$ and reversed $\omega_{\mathrm{r}}$ waves on the bias magnetic field $H_{0}$ at a fixed value of the pumping frequency $\omega_{\mathrm{p}}=9420 \mathrm{MHz}$. Dots correspond to the experiment (squares: signal frequency $\omega_{\mathrm{s}}$, and circles: frequency $\omega_{\mathrm{r}}$ of the reversed wave), and solid lines to the theory. Vertical dashed line indicates the maximum magnetic field $H_{\max }$, at which the WFR of MSSW is possible [43] (c). Experimental (symbols) and calculated (line) dependences of the reversed signal power on the input signal frequency in the regime of broadband input signal $\left(\Omega_{\mathrm{s}} / \Omega_{\mathrm{p}} \approx 3.5\right)$ [49]. Inset: the spectrum of the input signal (blue line) and the band of the parametric amplification (green line) $(d)$. The amplitude-frequency characteristic of a magnetostatic active filter for different pumping powers $P_{\mathrm{p}}\left(\square-P_{\mathrm{p}}=12 \mathrm{dBm}\right.$, $\left.\tau_{\mathrm{p}}=500 \mathrm{~ns} ; \circ-P_{\mathrm{p}}=26 \mathrm{dBm}, \tau_{\mathrm{p}}=100 \mathrm{~ns}\right)(e)[49]$

experimental evidence of the wave front reversal through a first-order three-wave parametric process.

The wave front reversal phenomena differ from simple wave reflection processes in many important respects (see, e.g., [44]). Whilst the direction of a reflected wave is determined by Snell's law, a reversed wave always propagates in the direction opposite to that of the source excitation. Moreover, under certain reversal conditions, the leading front of the source (incident) waveform is transformed into the trailing front of the reversed excitation, i.e. the time profile of the reversed waveform is an inverted version of the original. The effect of pulse profile time reversal has been well demonstrated in experiments involving two parametrically pumped pulses of different amplitudes $[39,45]$, and the phenomenon has also been observed in two-dimensional systems by Brillouin light scattering spectroscopy [46]. For the first time, G.A. Melkov and his colleagues conducted experimental studies of the wavefront reversal of slow short-wave dipole-exchange spin waves in thin films of yttrium-iron garnet (YIG) $[40,47,48]$. The investigations were carried out by the method of parametric interaction of spin waves with the electromagnetic pumping of the double frequency on a layout of the experimental section sketched in Fig. 2, a. The generation of dipole-exchange spin waves was a result of the two-magnon scattering of magnetostatic waves on inhomogeneities of the YIG film sample. The delay time of the dipole-exchange spin-wave signal exceeding $2.5 \mu$ s was experimentally obtained, and the effect of the defects made on the surface of the YIG film was studied. The phenomenon of double wavefront reversal was investigated by the method of cloning of the electromagnetic pump pulse [41]. This technique allows for an indefinitely long time to maintain the existence of magnetostatic waves greater than the level of the thermal noise in the YIG film by compensating of the propagation losses due to the action of a parametric pumping. G.A. Melkov and his colleagues for 
the first time conducted experimental and theoretical studies of wavefront reversal with parametric pumping under the conditions of anisotropic dispersion in the case of nonreciprocal surface magnetostatic waves [43]. To create an environment with anisotropic dispersion, it is sufficient to metallize the surface of the YIG film on one side only. In this case, the spectrum of magnetostatic waves looks as shown in Fig. 2, $b$. It has been found that the wavefront reversal has become possible only with the frequency conversion downward, if a direct wave propagates near the metallized surface of the film; otherwise, the wave does not exist. The maximum detected frequency shift was $800 \mathrm{MHz}$ (Fig. 2, c). It is established that, with a given external magnetic field, it is possible to stabilize the frequency of the inverted signal by changing the frequency of the input signal to $100 \mathrm{MHz}$, which can be used to create frequency stabilizers of the microwave signal. In work [49], G.A. Melkov and his colleagues investigated the nonresonant $\left(\omega_{\mathrm{s}} \neq \omega_{\mathrm{p}} / 2\right)$ wave front reversal of spin-wave pulses (carrier frequency $\omega_{\mathrm{s}}$ ) caused by a pulsed parametric pumping (carrier frequency $\omega_{\mathrm{p}}$ ) and showed that it can be effectively used for the microwave signal processing. When the spectral width $\Omega_{\mathrm{s}}$ of a signal is wider than the frequency band $\Omega_{\mathrm{p}}$ of the signal amplified by the pumping $\left(\Omega_{\mathrm{s}} \gg \Omega_{\mathrm{p}}\right)$, the nonresonant WFR can be used for the analysis of the signal spectrum (see Fig. $2, d$ ). In the opposite case $\left(\Omega_{\mathrm{s}} \ll \Omega_{\mathrm{p}}\right)$, the nonresonant WFR can be used for the active (with amplification) filtering of the input signal (Fig. 2,e).

One of the most interesting applications of the wave front reversal is the so-called reversal of the momentum relaxation proposed by G.A. Melkov and his colleagues $[47,50]$. Let us assume that the microwave signal pulse is supplied to the input antenna and excites a long-wavelength dipolar spin-wave in the ferrite film (magnetostatic wave), which is scattered in the course of propagation on the film inhomogeneities and is transformed into short-wavelength long-lifetime dipole-exchange spin waves. This transformation results in the disappearance of a signal wave. However, this process can be reversed via the parametric pumping. Applying it to the region with scatted dipole-exchange waves, it results in the wave front reversal of mentioned waves. They begin to propagate in the opposite direction, returning back to inhomogenitiesthe where they were "created". As a result of the inverse scattering process, they form a magnetostatic wave that propagates back to the input antenna. As we can see, the wave front reversal may be considered as the time reversal in this case, bringing back the signal wave scattered on ingomogenities. Moreover, multiply pumping pulses can be applied to the system resulting in the multiple acts of wave front reversal. In this way, one can control the appearance of the restored wave either on an input or output antenna.

The idea of the recovered signal dissipating into different modes in a magnetic system via a parametric pumping was further developed in work [51]. In this work, the signal propagating in a magnetic film dissipates into the so-called thickness spinwave modes, which are almost standing. The application of a pumping pulse to the system leads to the frequency-selective amplification of one of these standing modes. However, the nonlinear interaction in the magnon system ensemble should also be taken into account. The phase mechanism discussed above plays the governing role in the dynamics of a system and leads to the time shrinking of the recovered signal. The phase mechanisms result in the pumping being effective only over a certain finite time period, after which the saturation occurs, and the standing modes are suppressed by the so-called dominant spinwave group with the lowest relaxation parameter.

In addition, the nonresonant parametric recovery, in which the input signal frequency is detuned from a half of the pumping frequency, has been used to perform the spectral analysis of the stored microwave signal [52].

The parametrically pumped system of magnons can have nonconventional behavior, when the external pumpimg is switched off [53]. The monotonic decrease of the population of parametrically injected magnons is accompanied by an explosive behavior of the electromagnetic radiation at the magnon frequency. G.A. Melkov has developed a theory showing that this explosion is caused by a nonlinear energy transfer from parametrically driven short-wavelength dipolar-exchange magnons to a long-wavelength dipolar magnon mode effectively coupled to an electromagnetic wave.

The signal amplification and the wave front reversal by a parametric pumping open up a wide spectrum of different analog data processing devices based on magnetic materials $[48,49,54,55]$. Recent works of G.A. Melkov and his colleagues are devoted to the in- 
vestigation of different nonlinear and parametric processes in magnetic nanosystems [56-59]. A key feature of such systems is the discretization of the spinwave spectrum. Instead of the continuous spectrum of magnetic excitations that is typical of magnetic bulk samples or films in magnetic nanoelements due to the confinement, the spin-wave spectrum is discrete. This dramatically changes the dynamics of all nonlinear processes, they become nonresonant, and their threshold increases.

\section{Spin-Wave Solitons and Bullets}

One of the characteristic features of ferromagnetic materials is a strong nonlinearity of the magnetization dynamics. It is worth to note, that the origin of the magnetic nonlinearity is purely topological rather than dynamical. Namely, all important dynamic interactions in a magnetic medium, such as magnetodipolar and inhomogeneous exchange interactions, are linear (energy of a magnetic body is a quadratic function of the magnetization $\boldsymbol{M}$ ). At the same time, the length of the magnetization vector is preserved during the magnetization precession, $|\boldsymbol{M}|=$ const, which means that the phase space for the magnetization dynamics is a sphere rather than a plane. The intrinsic curvature of the spherical phase space is the interaction-independent origin of the strong magnetic nonlinearity.

The magnetic nonlinearity manifests itself in many physical phenomena, which both are of interest from the fundamental point of view and are important for practical applications. For instance, the magnetic nonlinearity enables the existence of various static magnetic textures such as domain walls, magnetic vortices, and skyrmions [60-64]. In the microwave domain, a nonlinear modification of spin-wave properties leads, in particular, to the formation of spatiallylocalized nonlinear wave packets - spin-wave solitons and bullets. The main characteristics of such objects are considered in this section.

Traditionally, the magnetization dynamics is considered in the framework of the Landau-LifshitzGilbert equation for the magnetization $\boldsymbol{M}$ [65]:

$$
\frac{d \boldsymbol{M}}{d t}=\gamma\left(\boldsymbol{H}_{\mathrm{eff}} \times \boldsymbol{M}\right)+\frac{\alpha_{\mathrm{G}}}{M_{\mathrm{s}}}\left(\boldsymbol{M} \times \frac{d \boldsymbol{M}}{d t}\right)+\boldsymbol{T}_{\text {add }} .
$$

Here, $\gamma \approx 2 \pi \times 2.8 \mathrm{MHz} / \mathrm{Oe}$ is the modulus of the gyromagnetic ratio, $\boldsymbol{H}_{\text {eff }}$ is the effective magnetic field related to the free energy of a ferromagnet $\mathcal{W}$ by
$\boldsymbol{H}_{\mathrm{eff}}=-\partial \mathcal{W} / \partial \boldsymbol{M}, \alpha_{\mathrm{G}}$ is the dimensionless Gilbert damping parameter, $M_{\mathrm{s}}=|\boldsymbol{M}|$ is the saturation magnetization, and the term $\boldsymbol{T}_{\text {add }}$ may describe the additional torques acting on the ferromagnet such as, e.g., the parametric pumping or spin-transfer torque.

The first term in Eq. (3) describes conservative interactions in a magnetic material and, usually, is much stronger than two other terms that describe dissipative effects. Solutions of the linearized conservative Landau-Lifshitz equation determine linear spinwave modes that can propagate in the medium. Such linear spin waves have been extensively studied and their properties are well understood now $[65,66]$. One of the peculiarities of spin waves in bulk ferromagnets is their anisotropic character, namely, the dependence of the dispersion relation $\omega=\omega(\boldsymbol{k})$ and other properties on the direction of the spin-wave wavevector $\boldsymbol{k}$ relative to the direction of the bias magnetic field.

Nowadays, the studies of spin-wave phenomena are typically performed in a thin-film geometry, i.e., using ferromagnetic films with thicknesses much smaller than in-plane sizes of the sample. This geometry provides easy means of electrical excitation and detection of spin waves and, also, allows one to directly observe the spatio-temporal evolution of spin waves using the Brillouin Light Spectroscopy (BLS) technique. In the thin-film geometry, only the lowest thickness spin-wave mode can be efficiently excited, i.e., the spin-wave system becomes effectively twodimensional. The natural spin-wave anisotropy translates into the dependence of the linear and nonlinear spin-wave properties on film's magnetization angle, which allows one to observe qualitatively different phenomena simply by rotating the direction of the bias magnetic field. Most of the experiments were performed in one of the three high-symmetry cases, in which different types of waves are excited [65]: (i) backward volume magnetostatic waves (BVMSW) propagating in an in-plane magnetized film along the direction of the bias field; (ii) magnetostatic surface waves (MSSW), which propagate perpendicularly to the bias field in an in-plane magnetized film, and (iii) forward volume magnetostatic waves (FVMSW) propagating in a normally magnetized films.

The analysis of the propagation of weaklynonlinear spin wave packets can be substantially simplified, by transforming the Landau-Lifshitz-Gilbert equation (3) to the equation for slowly varying complex envelopes [67]. Within this approach, one con- 
siders a spectrally narrow spin wave wavepacket centered at the wavevector $\boldsymbol{k}_{0}=k_{0} \boldsymbol{z}$ and propagating in a magnetic film lying in the $(y, z)$ plane. The slowly varying (in both space and time) spin-wave envelope can be defined as the inverse Fourier transform

$c(t, z, y)=\int \frac{d k_{z}}{2 \pi} \int \frac{d k_{y}}{2 \pi} c_{\boldsymbol{k}}(t) \times$

$\times \exp \left[i \omega_{0} t+i\left(k_{z}-k_{0}\right) z+i k_{y} y\right]$,

where $c_{\boldsymbol{k}}(t)$ is the amplitude of the spin-wave with wavevector $\boldsymbol{k}=k_{y} \boldsymbol{y}+k_{z} \boldsymbol{z}$, and $\omega_{0}=\omega\left(\boldsymbol{k}_{0}\right)$ is the central frequency of the wavepacket. Then Eq. (3) reduces to the dissipative nonlinear Schrödinger (NLS) equation [68]

$i\left(\frac{\partial c}{\partial t}+v_{g} \frac{\partial c}{\partial z}\right)+\frac{D}{2} \frac{\partial^{2} c}{\partial z^{2}}+\frac{S}{2} \frac{\partial^{2} c}{\partial y^{2}}-N|c|^{2} c=$ $=-i \Gamma c+f_{\text {add }}(c)$.

Here, $v_{g}=\partial \omega / \partial k_{z}$ is the group velocity of the spinwave wavepacket, $D=\partial^{2} \omega / \partial k_{z}^{2}$ is the dispersion coefficient, $S=\partial^{2} \omega / \partial k_{y}^{2}$ is the diffraction coefficient, $N \sim \partial \omega / \partial|c|^{2}$ is the nonlinear frequency shift coefficient, $\Gamma$ is the spin wave damping rate originating from the Gilbert damping term in Eq. (3), and $f_{\text {add }}(c)$ may describe additional forces created by the torque $\boldsymbol{T}_{\text {add }}$.

The nonlinear behavior of the spin-wave wavepacket is determined, mainly, by the dimensionality of the system and the interplay between the linear defocusing effects (dispersion $D$ and diffraction $S$ ) and nonlinearity $N$. The formation of solitons and bullets - localized nonlinear wavepackets - is possible, when these two trends compensate each other, i.e., when $D N<0$ or/and $S N<0$. This criterion of soliton formation is known as the Lighthill criterion [69].

The versatility of spin-wave characteristics in thin magnetic films allowed for the observation and investigation of practically all possible regimes of propagation and interaction of solitons and bullets. Below, we describe some of the most important experimental and theoretical results on spin-wave solitons and bullets.

In the simplest case, the propagation of spin-wave wavepackets is studied in a standard spin-wave delayline structure that uses a relatively narrow magnetic waveguide for the spin wave propagation. Typically, the width of such a waveguide is about 1-2 $\mathrm{mm}$, while its length is of the order of several $\mathrm{cm}$. In such a case, the lateral quantization of spin-wave modes across the waveguide width [70] excludes any diffraction-related effects, which corresponds to a one-dimensional wave propagation $(\partial / \partial y=0$ in Eq. (5)).

In the one-dimensional waveguide geometry, the Lighthill criterion of soliton formation $D N<0$ is satisfied for BVMSW and FVMSW waves in the dipolar region of the spin-wave spectrum. In the first experiments with spin-wave envelope solitons, however, dipole-exchange spin waves were used [7173]. Namely, these experiments were performed using thin films of yttrium-iron garnet (YIG). This dielectric ferrimagnet has record-low magnetic damping $(\Delta H \simeq 0.5 \mathrm{Oe})$. In the experiments, films with partially pinned surface spins were used, which led to a relatively strong hybridization of the lowest (dipolar) thickness spin-wave mode with higher-order exchange spin waves and very large values of the dispersion coefficient $D$ in the hybridization region. This allows one to use relatively long microwave pulses $(\tau>100 \mathrm{~ns})$ for the excitation of spin-wave solitons. The soliton formation was detected through the threshold increase of the transmitted power and the compression of the output spin-wave pulse.

Later, the envelope solitons of purely dipolar spin waves in YIG films with unpinned spins were observed and studied for both FVMSW [74, 75] and BVMSW [76-78] geometries. For the surface waves, the Lighthill criterion is not satisfied. In this case, the so-called "dark" solitons (stable localized dips on a strong continuous background) were experimentally observed [79, 80].

The magnetic damping in YIG films, although being much smaller than in other magnetically ordered materials, has substantial impact on the envelope soliton formation and propagation [81]. Therefore, a lot of attention was paid to the question of the spin-wave soliton amplification. For the first time, this problem was addressed in Ref. [82] with the use of the parametric interaction of spin waves with a parallel parametric pumping at the double frequency. Although the soliton amplification gain achieved in Ref. [82] was rather small (around $1 \mathrm{~dB}$ ), improvements of the proposed method demonstrated later a possibility of the parametric amplification of single-soliton pulses with substantial gains $K_{s}>10 \mathrm{~dB}$ [45, 83-86].

It should be noted that the amplification of envelope solitons faced specific difficulties that are absent for linear pulses $[85,86]$. Namely, the balance between 
the nonlinear and dispersive effects determines the relation between the soliton amplitude $A$ and its spatial size $\ell$ :

$A=\sqrt{|D / N|} \ell^{-1}$.

If a soliton is amplified without any shape distortions, and if the amplitude amplification gain $\kappa=A^{\prime} / A$ is smaller than $3 / 2$, the amplified pulse restores its soliton profile during the further propagation in the medium. This soliton restoration is accompanied with compression and additional increase of the soliton amplitude, yielding the soliton amplitude amplification $K_{s}=2 \kappa-1$. If, however, the initial amplification is relatively strong, $\kappa>3 / 2$, the amplified pulse splits into two or more solitons. This means that the maximum single-soliton amplification is limited by $6 \mathrm{~dB}$. This problem of effective single-soliton amplification was solved in $[85,86]$, by using the parametric interaction of spin wave solitons with a strongly localized nonstationary parametric pumping (see Fig. 3).

The parametric interaction of spin waves was also used to study the formation of one-dimensional envelope solitons $[87,88]$ and two-dimensional bullets [46] from linear input wavepackets.

Another interesting direction in spin-wave soliton researches is the study of the self-generation of soliton trains and other nonlinear wave patterns in active spin-wave rings. An active ring represents a spinwave delay line with active feedback, i.e., the output spin-wave signal detected by a microwave antenna is amplified and, possibly, filtered, and then is supplied back to the input antenna. For small amplification gains, the active ring generates quasimonochromatic spin-wave signal, which transforms to various nonlinear patterns with an increase of the gain. Using such setups, the formation of regular soliton trains $[89,90]$, self-generation of two-dimensional bullets [91], spontaneous symmetry breaking of soliton trains [92], chaotic soliton dynamics [93, 94], fractal dynamics [95], Fermi-Pasta-Ulam recurrence [96], and other interesting phenomena were observed.

When the width of an YIG waveguide is increased, the transition from the one-dimensional spin-wave dynamics to a two-dimensional one occurs. Such studies were performed, mostly, in BVMSW geometry, for which both the dispersion and diffraction satisfy the Lighthill criterion, and the formation of two-dimensional localized structures - bullet, is expected. The crossover to the effectively two-

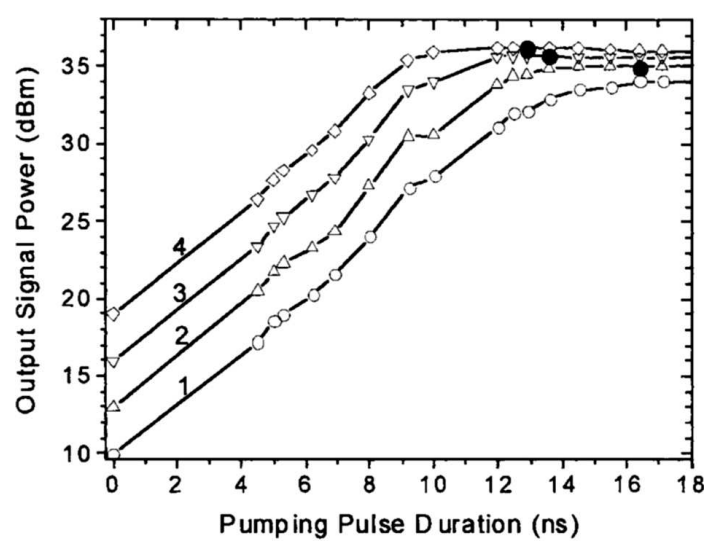

Fig. 3. Amplification of spin-wave pulses by a strongly localized nonstationary pumping for different input signal powers: (1) $10 \mathrm{~mW}$, (2) $20 \mathrm{~mW}$, (3) $40 \mathrm{~mW}$, (4) $80 \mathrm{~mW}$ (single-soliton pulse). The black dots indicate the points, where the formation of a second soliton took place. The single-soliton amplification $K_{s}>15 \mathrm{~dB}$ was achieved. (from Ref. [86])
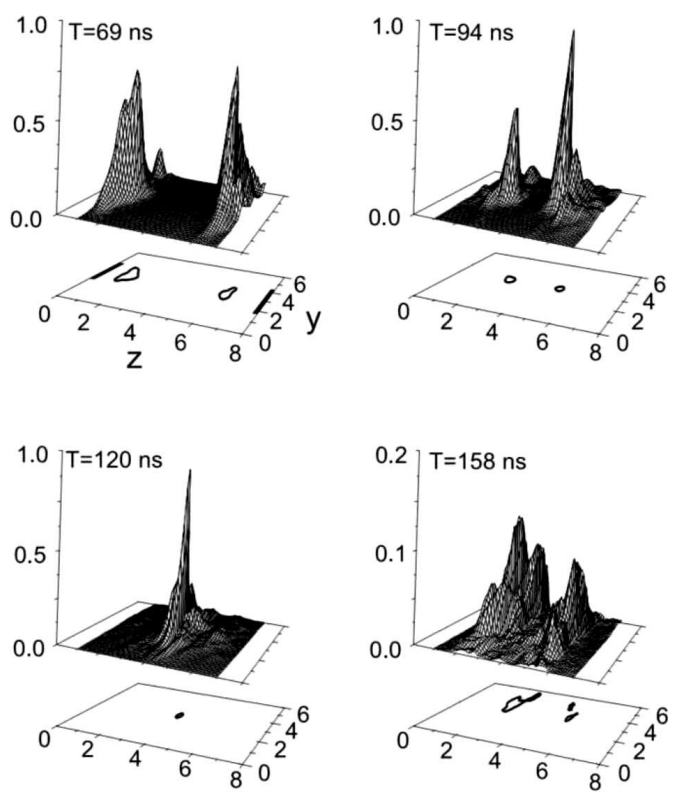

Fig. 4. Collision of two-dimensional spin-wave bullets in a wide YIG film. The upper (lower) frames show the twodimensional intensity distribution (cross-section at the halfmaximum level) of the propagating wave packets for four different values of the propagation time as indicated (from Ref. [101])

dimensional system is found to be power-dependent $[97,98]$ and leads, eventually, to the formation of highly-nonlinear bullet modes [99, 100]. In contrast with one-dimensional solitons, two-dimensional bullets are unstable and collapse [101-103] (see Fig. 4). 
A different type of spin-wave bullets is realized in nano-scale current-driven metallic nano-contacts [104-108]. In this case, the current-induced spintransfer torque [109-111] compensates the magnetic damping (which can be described by the effective negative damping term $f_{\text {add }}=\Gamma_{-}\left(|c|^{2}\right) c$ in Eq. (5), and the standing spin-wave bullet with $k_{0}=0$ and $v_{\text {gr }}=0$ is excited. Properties of such bullets and other current-driven dynamic solitonic structures are described in details in the recent review [112].

\section{Magnon Gases, \\ Bose-Einstein Condensates and Supercurrents}

\subsection{Bose-Einstein condensation}

Bose-Einstein condensation [113-115] is one of the most exciting and intriguing quantum phenomena. Generally speaking, this process can be described as the coalescence of bosons (including real atoms and Cooper pairs of fermions, and quasiparticles of different nature) into the same quantum state and, thus, constituting a specific state of matter described by a single wave function - a Bose-Einstein condensate (BEC). The prerequisite of the BEC formation is exceeding the threshold density of the particles at the given temperature. This requirement justifies the necessity of cryogenic conditions for the observation of the BEC in a gas of real atoms [116]. Besides of the fundamental importance of the BEC-related phenomena such as superconductivity and superfluidity, they are thought to be at the frontline for the potential use in the next generation of electronic devices. A major challenge in this area is the realization of the room-temperature macroscopic quantum state computing.

\subsection{Room-temperature magnon Bose-Einstein condensation}

The first theoretical predictions that a BEC can occur at higher (or even at ambient) temperatures were made by Fröhlich in 1968 [117]. On the way to ambient-temperature BECs, the approach is to use quasiparticles instead of real atoms. Quasiparticles are excitations of a many-body system, the vast majority of which are bosons and therefore can form a BEC. Compared to the real atoms, quasiparticles have many orders of magnitude smaller effective mass, which decreases their density threshold for the BEC observation. However, unlike real atoms, quasi- particles decay and decohere with time, making the experimental observation of their BEC very challenging. In order to compensate the natural decay, it is necessary to externally add an excess of quasiparticles. In such a case,the condensation is possible, if the flow rate of energy pumped into the system exceeds a critical value.

Magnons, being the quanta of spin waves, are bosons. Thus, in the thermal equilibrium, they can be described by the Bose-Einstein statistics with the zero chemical potential and a temperature-dependent density. At cryogenic temperatures, the magnon BEC was observed in a superfluid ${ }^{3} \mathrm{He}$ [118]. Theoretical foundations for the room-temperature magnon BoseEinstein condensation were developed in 1989 by Kalafati and Safonov $[119,120]$. These predictions combined with the rich experience of G.A. Melkov and co-authors on the parametric excitation of magnons $[65,121]$ and the Brillouin light scattering (BLS) spectroscopy technique [122] resulted in the first experimental observation of a magnon $\mathrm{BEC}$ at room temperature in Yttrium Iron Garnet $\left(\mathrm{Y}_{3} \mathrm{Fe}_{5} \mathrm{O}_{12}\right.$, YIG) [123]. There were important preconditions for this observation. The first is the selection of the material. YIG films provide a very long spin-lattice relaxation time of up to $1 \mu \mathrm{s}$. Thus, the lifetime of magnons is sufficiently longer than the magnon-magnon thermalization time due to the two- and four-magnon scattering processes, which can be as low as 10-100 ns. As these scattering events conserve the number of magnons, a quasiequilibrium state for the magnon gas can be realized with a nonzero chemical potential. The influence of three-magnon processes can be significantly reduced by a proper selection of the magnetic field and the magnon excitation frequency. The second precondition is the fast (on a time scale of several orders of magnitude shorter than the relaxation time) creation of an overpopulation of the magnon spectrum. It was realized with the use of the parametric pumping, which offers an efficient way to excite a large number of magnons in the low-frequency part of the magnon spectrum, which then can thermalize into magnons with the minimum frequency. Using this technique, a pumped magnon density of above $10^{19} \mathrm{~cm}^{-3}$ can be reached $[124,125]$. Although this density is much smaller than that of thermal magnons at room temperature $\left(\sim 10^{22} \mathrm{~cm}^{-3}\right)$, this increase is sufficient to trigger the BEC formation.

ISSN 2071-0194. Ukr. J. Phys. 2019. Vol. 64, No. 10 
Another experimental challenge was to detect magnons in the BEC. The magnon spectrum of an inplane magnetized YIG film is highly anisotropic in relation to the direction of magnon's wavevector. This anisotropy originates from the dipolar interaction and together with the isotropic influence of the exchange interaction leads to the appearance of the global energy minimum at the longitudinal branch of the magnon spectrum at a relatively high wavenumber $q=4 \times 10^{4} \mathrm{rad} \mathrm{cm}^{-1}$ (see Fig. 5, b). This fact makes the conventional electromagnetic detection of the condensed magnons hard to be realized. However, the optical detection using BLS (via the inelastic scattering of photons on magnons) gives the necessary wide-range wavevector sensitivity together with decent frequency resolution. The BLS experiments were performed with time resolution, wherein the start of the pumping pulse triggers a stroboscopic clock for a counter of scattered photons. The main elements of the typical experimental setup for a BLS experiment are sketched in Fig. 5, $a$. Figure $6, a$ shows the BLS spectra of the pumped magnons recorded at different delay times after the switching-on of the pumping pulse (duration $1 \mu \mathrm{s}$, repetition period $20 \mu \mathrm{s}$ ). Under the pumping action, the number of magnons increases in the system with time. After $300 \mathrm{~ns}$, the measured spectrum was already not possible to be fitted with the use of the Bose-Einstein distribution. The only way to achieve an agreement with the experiment was to include a singularity peak in the fitting procedure at the frequency of the bottom of the magnon spectrum, which strongly evidenced the BEC formation.

\subsection{Magnon BEC formation studies}

In the following several years, the studies around the magnon BEC were focused on the mechanisms of its formation. The magnon thermalization dynamics has been studied in details as a function of the applied microwave pumping power, defining a threshold of the BEC formation using BLS techniques [127, 128], as well as using microwave measurements [129]. The BEC formation has been found to be almost independent of the frequency spectrum of the pumping source and is solely determined by the density of magnons [130]. In the same time, the rather indirect evidence of the magnon BEC coherence triggered a further theoretical investigation of this phenomenon [131, 132].

Most of the studies of quasiparticle BECs have implicitly assumed that the injected particles thermal-

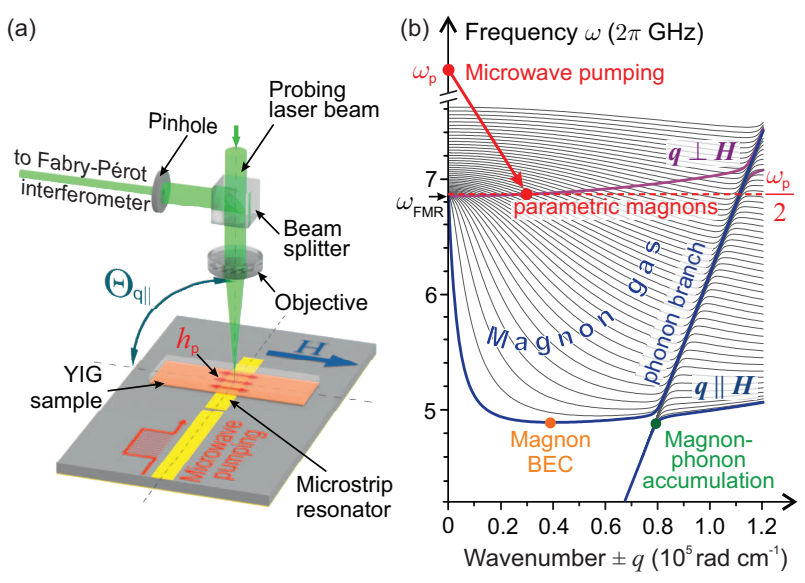

Fig. 5. Schematic illustration of the typical BLS experimental setup [135]. The resonator concentrates the applied microwave energy and induces a pumping microwave Oersted field $\boldsymbol{h}_{\mathrm{p}}$ oriented along the bias magnetic field $\mathbf{H}$, thus realizing conditions for the parallel parametric pumping. The probing laser beam is focused onto a YIG film placed on the top of the microstrip resonator. The light inelastically scattered by magnons is redirected to a Fabry-Pérot interferometer for the frequency and intensity analysis. The wavenumber-selective probing of magnons with wavevectors $\mathbf{q} \| \mathbf{H}$ is realized by varying the incidence angle $\Theta_{q \|}$ between the field $\mathbf{H}$ and the probing laser beam $(a)$. The magnon-phonon spectrum of a $6.7 \mu$ m-thick YIG film is calculated for $H=1735$ Oe. 47 thickness modes with $\mathbf{q} \| \mathbf{H}$ are shown. The upper thick curve shows the most effectively parametrically driven [126] lowest magnon mode with $\mathbf{q} \perp \mathbf{H}$. The arrow illustrates the magnon injection to the frequency $\omega_{\mathrm{p}} / 2$ slightly above the ferromagnetic resonance frequency $\omega_{\mathrm{FMR}}(b)$

ize over the entire spectrum and that the sole role of the pumping is to increase the quasiparticle density and, thus, the chemical potential of the gas. However, such an assumption is a crude oversimplification. The development of the wavevector-resolved BLS spectroscopy techniques applicable to the study of a longitudinal branch of the magnon spectrum $[128,133-136]$ gave the necessary insight into the mechanisms of the magnon thermalization and condensation. Observations of the transitional dynamics of gaseous and condensed (BEC) components of a pumped magnon gas (see Fig. 6, b) [136] were found to be qualitatively incompatible with a uniform thermalization model. As can be seen from the measured time-dependent distribution function of a magnon gas in the energy-phase space, parametrically pumped magnons thermalize and create a quasiequilibrium state mainly in a narrow low-energy part of the mag- 

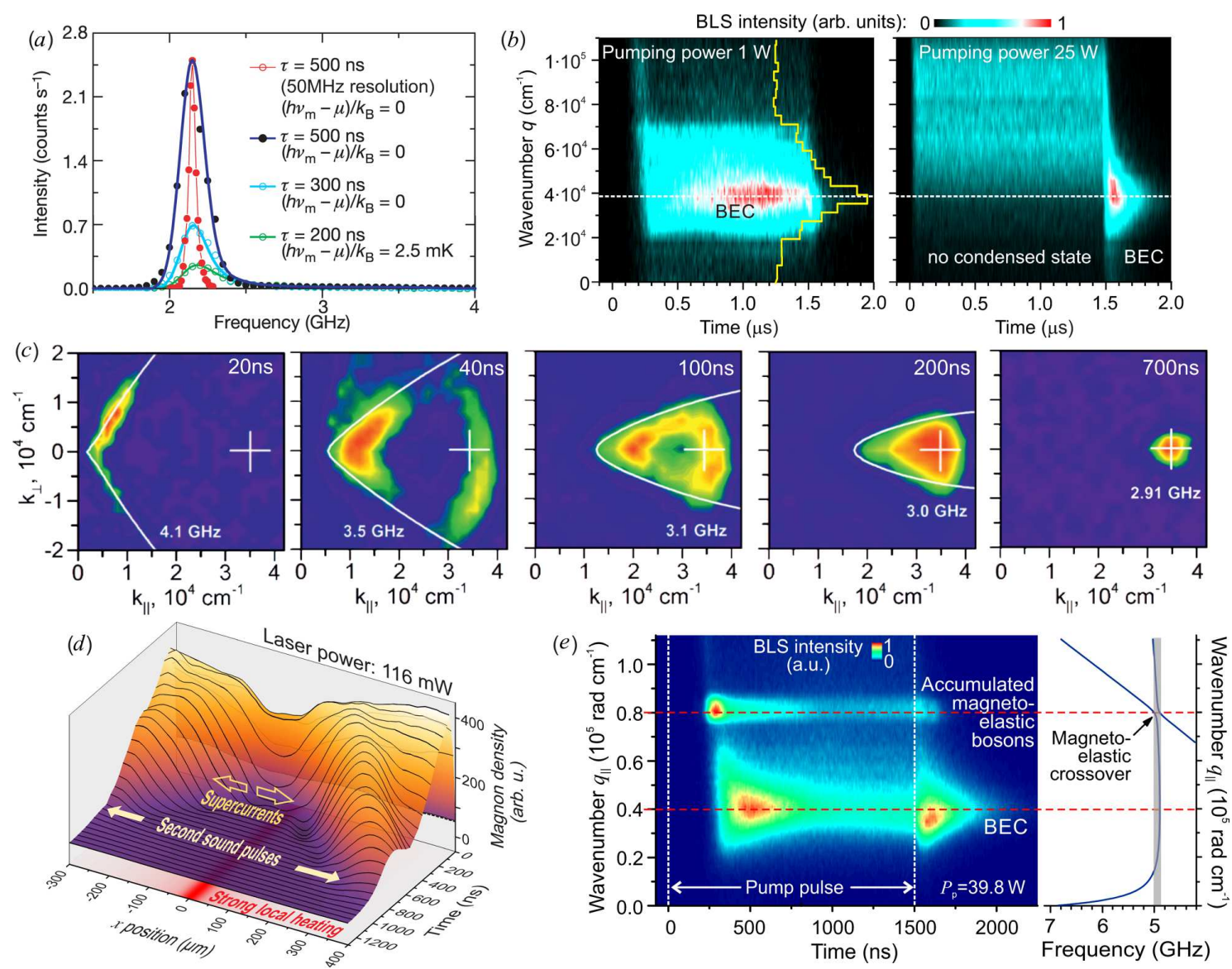

Fig. 6. BLS spectra from pumped magnons at different delay times. Dots are the measured data, and solid lines indicate the fitting by the Bose-Einstein distribution function. Red circles represent the data obtained with a resolution of $50 \mathrm{MHz}$. Red line is a guide for the eye, connecting the red circles. [123] $(a)$. The time evolution of the BEC of magnons in the wavenumber space. The BLS intensities are measured in a frequency band of $150 \mathrm{MHz}$ near the bottom of the magnon spectrum for different magnon wavevectors along the magnetization direction. [136] (b). The color-coded representation of the normalized BLS intensity measured for different two-dimensional magnon wave vectors. The colormap reflect the distributions of the magnon density in the wave-vector space. Subsequent panels correspond to different delays after the start of the pumping pulse, as indicated. The white lines indicate the magnon spectrum in the form of constant-frequency contours, and the cross indicates the position of the bottom of the magnon spectrum [133] (c). Time-space population diagram of the magnon Bose-Einstein condensate with a strong localized heating. It results in the formation of dense condensate humps (Bogoliubov waves), which propagate in opposite directions along the microstrip and perpendicularly to the bias magnetic field through the cold magnon BEC over distances of hundreds micrometers [144] $(d)$. The left panel shows the temporal dynamics of the magnon gas density with a pronounced accumulation of magnetoelastic bosons for the broad pumping area $500 \mu \mathrm{m}$ in width. The right panel shows the magnon-phonon dispersion for the given experimental conditions [145] $(e)$

non spectrum at relatively low pumping powers (left panel in Fig. 6, $b$ ). The yellow line in Fig. 6, $b$ shows the magnon distribution function at $1.24 \mu$ s after the pumping pulse $(1.5 \mu$ s long) was applied. This observation clearly indicates the formation of a magnon BEC during the pumping pulse. However, at a higher pumping power (right panel in Fig. 6, $b$ ), the situation drastically changes: this quasiequilibrium spectral region is now characterized simultaneously by a very low average magnon energy (of the order of the energy of pumped magnons) and a very high effective temperature $\left(T_{\mathrm{m}} \simeq 30000 \mathrm{~K}\right)$. Moreover, a strikingly unexpected dynamics of the magnon BEC is observed: the density of the condensate sharply in- 
creases immediately after the external magnon injection is switched-off. This behavior can be explained with a nonlinear "evaporative supercooling" mechanism [136]. This mechanism, which is based on fourmagnon scattering processes, couples the low-energy magnon area overheated by the pumping with all the other thermal magnons. The magnons that scatter to upper frequencies remove the excess heat and cool down the magnon gas. As a result, the BEC formation is enhanced.

With the help of a $2 \mathrm{D}$ wavevector-resolved BLS spectroscopy, one can track the magnon cloud on its way to the condensate [133] (see Fig. 6, c). In this case, the injection point of parametric magnons was tuned to be in the dipolar area of the spin-wave spectrum (below the Ferromagnetic Resonance (FMR) frequency), which is easily accessible by the wavevector-resolved BLS spectroscopy based on a wideaperture lens and the moving diaphragm approach [137]. The subsequent panels in Fig. 6, $c$ demonstrate snapshots of the magnon density distribution during the thermalization process recorded at different delays after the start of the pumping pulse. The dipoledominated magnons thermalize to the bottom of the spin-wave spectrum rather slowly (150-200 ns) and are distributed along the line of equal frequency. In contrast, the exchange-dominated magnons reach the bottom of the spectrum already in the first $40 \mathrm{~ns}$ after the start of the pumping pulse and have a more complicated redistribution. Finally, after $\approx 700 \mathrm{ns,} \mathrm{the}$ magnon distribution narrows, as the BEC is formed.

Later, the contributions of both dipolar and exchange thermalization channels were studied in details in Ref. [134] by means of the improved widewavevector-selective BLS setup. It was found that the magnon population in these thermalization channels strongly depends on the applied microwave pumping power. The observed magnon redistribution between the channels is caused by a downward frequency shift of the magnon gas spectrum due to a decrease of the saturation magnetization in the course of injection of parametrically pumped magnons. Further, in order to track the magnon cloud, while it thermalizes through the exchange channel, the pumping pulse was significantly shortened to $100 \mathrm{~ns}$ [128]. This modification allowed one to reveal that the condensation is preceded by the conversion of initially pumped magnons into a second group of frequency-degenerated magnons, which appear due to the parametrically stim- ulated scattering of the initial magnons to a shortwavelength spectral region. In contrast to the originally pumped magnon group, in which the wave vectors are orthogonal to the wave vectors of the magnons at the lowest energy states, those secondary magnons can effectively scatter to the bottom of the spectrum and condense there.

The magnon spectrum is rather anisotropic, which opens wide opportunities for various multimagnon processes, which can enhance the BEC formation $[38,138]$. Recently, the magnon BEC formation process was studied in the regime of strong direct population of the bottom of the magnon spectrum [139] the so-called Kinetic Instability (KI) [140]. The KI process takes place, when the initial group of parametric magnons is generated in the area of the spectrum, from where the direct four-magnon scattering to the minimum of the spectrum is allowed by the energy and momentum conservation laws. The study of a magnon BEC was performed in a wide range of external magnetic fields. The externally pumped magnons were transferred toward the condensed state via two distinct channels: a multistage thermalization cascade of the weak-wave turbulence and a one-step KI process. It has been shown that opening the KI channel leads to the formation of a much denser magnon condensate.

Creating the magnon overpopulation by the parametric pumping is not the only way to achieve a BEC. As follows from the fact that magnons naturally decay to the phonon bath, the magnon BEC cannot be achieved by a quasistatic lowering the temperature, as the chemical potential in such a case will be always equal to zero. However, if the temperature is greatly reduced much faster than the characteristic time scale of the magnon-magnon scattering, the excess thermal magnons will increase the chemical potential and might form a BEC. In macroscopic samples as used in the previous experiments, it is very hard to create preconditions for the rapid cooling due to the limited thermal conductivity of YIG and its substrate. However, in nanostructures with a reduced volume of the sample, the characteristic time scales for the cooling can be made as short as a few nanoseconds [141]. The magnon population around the low-frequency part of the spectrum in a nanostructured waveguide was monitored by means of timeresolved microfocused BLS. After the initial heating of the nano-YIG layer by a dc pulse through the at- 
tached Platinum heater layer, an excess of thermal magnons is created. After switching-off the heater, the system rapidly cools down, and the pronounced peak of magnon density is formed at the lowest frequency in the spectrum. Fitting the observed magnon populations with the Bose-Einstein distribution confirmed the formation of a magnon BEC [141].

\subsection{Magnon supercurrents, second sound, and Bogoliubov waves}

By analogy to superconductivity and ultracold atomic systems, quasiparticle BECs reveal the macroscopic quantum phenomena like supercurrents, Josephson effects, second sound, and Bogoliubov waves. In order to be observed, these effects require a coherent interaction between two spatially separated BECs or a presence of a phase gradient in the condensate's wavefunction. Starting from the discovery of a magnon BEC, the intense work is being performed to control the magnon BEC localization and phase. One of the first demonstrations of two separated magnon BECs was done in Ref. [142]. By making use of different excitation geometries - namely, a thin wire and a microstrip, the different spatial localization of the condensate was achieved. Two spatially separated condensate clouds were simultaneously created in a magnetic film in the case of the $500 \mu \mathrm{m}$-wide microstrip resonator. However, no interaction between condensates was observed that time.

In the geometry of in-plane magnetization conventional for the magnon BEC observation (see Fig. 5), the strong magnetic dipole-dipole interaction results in two symmetric minima in the frequency spectrum. Consequently, a system of two BECs with opposite wavevectors is created. These condensates can form a specific interference pattern, which can be revealed by means of the microfocused BLS technique [143]. The mutual coherence of two condensates leads to the establishment of a standing wave with distinct signatures of phase slips in the form of quantized vortices.

In order to explore further the coherence of a magnon BEC and make use of it to develop novel wavebased computing schemes, one needs a mechanism of local control over condensate's phase. One of such methods is to create a localized frequency shift of the magnon dispersion by the local heating [144]. The experiment was done in a classical geometry suitable for the observation of a magnon BEC magnetiza- tion in a $6.7 \mu$ m-thick YIG film (see Fig. 5, a). The temporal evolution of the magnon BEC formed in a parametrically populated magnon gas was studied by means of time- and wavevector-resolved BLS spectroscopy. The thermal gradient in the condensate was created by a focused probing laser beam. To achieve the control over the heating, the laser beam was chopped by an acousto-optical modulator. It has been found that the local heating at the focal point of a probing laser beam leads to the excessive decay of a magnon BEC, which is associated with the outflow of condensed magnons driven by a thermal gradient. The magnon BEC flow occurs in the opposite to a seemingly obvious direction toward a potential well created in the hot region, which confirms the phasedependent nature of the observed phenomenon - magnon supercurrent [144].

The first observation of a magnon supercurrent [144] was rather indirect. In the following experiment, the heating and probing beams were separated in order to directly investigate the propagation of magnon supercurrent pulses [145]. In that experiment, the heating was provided by a continuous $405 \mathrm{~nm}$ laser, which is well separated from the $532-\mathrm{nm}$ wavelength of a probing laser and also well absorbed by an YIG film. By shifting the detection point across and outside the heated area, the spatio-temporal diagram of the BEC propagation was measured (see Fig. 6, d). After the formation of a BEC at the end of the pumping pulse [136], a clearly visible well in the magnon population is formed. In the same time, we observe magnons, which are being pushed out by magnon supercurrents and form distinct peaks at the sides of the heated area after $\sim 200$ ns. It is remarkable that these peaks do not stay at the edge of the heating area (as it is expected from the supercurrent-driven transport), but start to propagate with an initial group velocity of $410 \mathrm{~m} \cdot \mathrm{s}^{-1}$. As the condensate propagates, its population decreases, since magnons naturally decay. The group velocity of the BEC peaks also decreases to $361 \mathrm{~m} \cdot \mathrm{s}^{-1}$ after $\sim 1200 \mathrm{~ns}$ of the propagation time. This phenomenon can be understood as the excitation of a new type of second sound: Bogoliubov waves in the magnon BEC condensate. As the population of the BEC decreases, the group velocity $c_{s}$ decreases as well, as a result of the dependence of the dispersion of Bogoliubov waves on the condensate's population $N_{c}: c_{s} \propto \sqrt{N_{c}(t)}$ (which follows from the characteristics of magnon-magnon interac-

ISSN 2071-0194. Ukr. J. Phys. 2019. Vol. 64, No. 10 
tions [146]). Despite the decay and a decrease of the group velocity, the propagation was detected over the macroscopic distances up to more than $600 \mu \mathrm{s}$. The discovered magnon second sound differs from the second sound in dielectrics, in which the phonons can be described in terms of their occupation numbers only, not taking their phases into account. It also differs from the second sound in superfluid ${ }^{4} \mathrm{He}$ and in the $\mathrm{BEC}$ of diluted atomic systems, where the wave function describes the distribution of real atoms and not of quasiparticles, as in the case of magnons. From a practical point of view, the three observed phenomena: (i) the transition from the supercurrent-type to the second-sound-type propagation regime, (ii) the excitation of second-sound-pulses, and (iii) the possibility of a long-distance spin-transport in the magnon BEC, has already paved a way for the application of magnon macroscopic quantum states for low-loss data transfer and information processing in perspective magnon spintronic devices. There are no doubts that the magnon second sound requires further detailed experimental and theoretical investigations. For example, the excitation of a second sound waves in a dense magnon gas was recently realized by using local magnetic fields generated by current pulses in microstructured wires, as has been reported in Ref. [147].

\subsection{Bottleneck accumulation of magnetoelastic bosons}

So far, considering a magnon BEC and its excitations, the magnon-phonon interaction was taken into account only as a general decay mechanism of magnons to the lattice. However, the magnonphonon scattering processes can significantly modify the scenario of thermalization of a pumped magnon gas. The magnon dispersion curvature in a vicinity of the hybridization between magnon and transverse phonon modes experiences strong modifications (see Fig. 5, b) [148]. This leads to a significant increase in the group velocity of magnons and magnon-phonon hybrid quasiparticles. The manifestations of the magneto-elastic interaction can be already marked in the left panel of Fig. $6, b$ - there is a slight decrease in the detected magnon density in the initial magnon thermalization stage around $q_{\|} \approx 8 \times 10^{4} \mathrm{rad} \cdot \mathrm{cm}^{-1}$.

In an experiment similar to that earlier described in the section devoted to the magnon BEC forma- tion and reported in Ref. [136], the increase in the width of the pumping region to $500 \mu \mathrm{m}$ leads to drastic changes of the thermalization behavior (see Fig. 6, e) [135]. After about 200 ns, first magnons appear in the exchange part of the spectrum near $q_{\|}=10 \times 10^{4} \mathrm{rad} \cdot \mathrm{cm}^{-1}$. Then the pronounced peak at the hybridization point at $q_{\|}=8 \times 10^{4} \mathrm{rad} \cdot \mathrm{cm}^{-1}$ appears. Note that the observed peak is as narrow as the wavenumber resolution of the experimental setup, i.e., $\pm 0.2 \times 10^{4} \mathrm{rad} \cdot \mathrm{cm}^{-1}$. During the further time evolution, the magnons occupy the energy states around the global energy minimum $q_{\|}=$ $=4 \times 10^{4} \mathrm{rad} \cdot \mathrm{cm}^{-1}$ and tend to form there a BEC. It is worth to note that the population peak is visible, only if a sufficiently wide microstrip resonator is used to create the pumping microwave field. The accumulation occurs into the magnon-phonon hybridization region, where quasiparticles possess rather high group velocities. The widening of the width of the microstrip reduces their leakage losses from the pumping area. It is worth to mention that the magneto-elastic peak starts to be visible at pumping powers approximately ten times smaller than the threshold of the BEC formation.

Obviously, the mechanism, which is responsible for the observed accumulation, must significantly differ from that in the conventional BEC, as no energy minimum exists in this region (see the dispersion panel in Fig. 6, e). To understand the observed phenomenon, we should consider the physics of the scattering processes leading to the thermalization of the parametrically driven magnon gas. The excitation of parametric magnons (see Fig. 5, b) populates the isofrequency surface at a half of the pumping frequency. The essential part of these magnons are in the exchange region of the spectrum, where the four-magnon scattering processes dominate over other types of interactions. This distributes parametric magnons over the entire wavevector space in such a way that the magnon number flux is mostly directed toward lower frequencies. The fastest track for the exchange magnons toward the bottom of their spectrum is through the lowest magnon mode with wavevectors parallel to the external bias field [135]. During the thermalization process, magnons reach the area of hybridization between the lowest magnon mode and the transverse phonon branch, gradually converting into hybrid magnon-phonon quasiparticles. As phonons are very linear particles and does not support a nonlinear 
scattering, these quasiparticles cannot scatter further down by frequency along the phonon branch. In the same time, their scattering ability to the other (higher by frequency) magnon and magnon-phonon branches is also limited, as the scattering rate is proportional to the populations of the interacting quasiparticle's spectral groups. This bottleneck in the scattering efficiency leads to building up a high population of the hybridization region of the spectrum. It is necessary to note that the bottleneck accumulation of hybrid magnetoelastic bosons is exclusively related to the variable scattering interaction of quasiparticle hybrids and, thus, should not be mixed with a "bottleneck" in a relaxation chain from an externally excited quasiparticle state to the phonon bath. In our case, the latter effect is a prerequisite for an increase in the density of externally pumped gaseous magnons above the thermal level. The magnon-phonon bottleneck accumulation phenomenon is not unique for this particular system and can occur in any multicomponent gas mixture of interacting quasiparticles with significantly different scattering amplitudes.

\section{Magnon Caloritronics}

Spin caloritronics is a dynamically emerging field of research, which investigates the interplay between spin- and heat-based transport phenomena [149]. The observation of the longitudinal spin Seebeck effect (LSSE) in magnetic insulators [150, 151] demonstrates the crucial role of collective magnetization excitations, i.e., spin waves and their quanta, magnons, in spin-caloric transport processes, and illustrates the conceptual distinction between this phenomenon and the conventional thermoelectric generation effect. The strong impact of magnons on the LSSE has also been reported for magnetic semiconductors [152]. Contemporary theories in the field of spin caloritronics consider magnons as one origin of spin-dependent phenomena in magnetic insulators $[153,154]$.

Nowadays, the interaction between phonon and magnon subsystems of a magnetic insulating medium is a hot topic of researches in modern magnon caloritronics, which is the subfield of spin caloritronics. Magnon caloritronics provides ideas of and methods about how to influence and to control magnon currents by heat currents and vice versa. The complexity of magnon spectra, their dependence on the orientation of a bias magnetic field, and the existence of both bulk and surface magnon modes make the investigations very complex and intriguing [155]. Yttrium iron garnet (YIG) ferrite films epitaxially grown on a gadolinium gallium garnet (GGG) substrate are ideal candidates for the magnon-caloritronic studies due to the extremely low magnetic damping [155,156].

Magnon properties, like the density, frequency, wave-vector, and group velocity, are strongly dependent on the temperature mainly due to the temperature dependence of the saturation magnetization of a magnetic media. Therefore, these parameters can be tuned by changing the thermal environment. Additionally, the magnon damping can be manipulated using thermal gradients across the thickness of yttriumiron-garnet/platinum structures, as it was shown by M.B. Jungfleisch et al. [157]. It was demonstrated by the spin pumping and by microwave techniques that the damping is either increased or decreased depending on the sign of the applied temperature gradient. Even the amplification of propagating dipolar magnons by a thermal spin-transfer torque created by spin currents, which are generated through the spin Seebeck effect (SSE), was reported [158]. Recently, the role of a damping in the spin Seebeck effect in yttrium iron garnet thin films was studied experimentally [159]. It was demonstrated that a decrease in the intrinsic damping of the magnetic material gives rise to an increase in the SSE coefficient, and this response also shows a quasilinear behavior.

The influence of a thermal gradient on the propagation properties of externally excited dipolar spin waves in an YIG waveguide is investigated by the representatives of G.A. Melkov's scientific school [160]. It is shown that spin waves propagating toward a colder region along the magnetization direction continuously reduce their wavelength. The reflection of spin waves was demonstrated in the case of propagation toward a hotter region, since their existence in a region above a critical temperature is prohibited. Based on this, it is shown that the thermal gradient applied to a YIG magnonic crystal along the propagation direction leads to a frequency shift and a substantial modification of the transmission characteristics of propagating backward volume and surface magnetostatic spin waves [161]. These influences of the temperature on spin-wave properties are mainly caused by a change in the saturation magnetization and yield promising opportunities for the manipulation of spin waves in magnon-caloritronic applications. For 
instance, an approach for producing fully tunable, two-dimensionally structured magnetic materials is proposed and realized [162]. Thermal landscapes in a magnetic insulator were created using a laser that results in modulations of the saturation magnetization and in the control over spin-wave characteristics. This method is demonstrated by the realization of fully reconfigurable one- and two-dimensional magnonic crystals [162].

Together with the angular momentum, magnons also carry the energy. Thus, the magnon-mediated heat transport in magnetic insulators constitutes another topic of magnon-caloritronic researches. A few promising achievements attained by the representatives of G.A. Melkov's scientific school in this direction are mentioned below. The magnetically controlled heat flow carried by a magnon current in a magnetic insulator was observed, and the magnoninduced heat conveyer effect has been explored [163]. The direction of the flow can be switched by changing an applied magnetic field. This phenomenon is understood as the unidirectional energy transfer by the excitation of nonreciprocal spin-wave modes. It can be applied potentially to the construction of a microwave heat transport system for manipulating the heat over length scales of several millimeters in solids, thereby opening the door to a heat technology based on spin currents.

The discovery of the spin Seebeck effect (SSE) in ferromagnet/nonmagnetic-metal bilayers [164] opened the key direction of investigations in spin caloritronics. The further observation of the longitudinal spin Seebeck effect in magnetic insulators demonstrated the essential role of magnons in this process $[150,151]$. Up to now, the LSSE was found in a large variety of magnetic insulating materials covered with the different nonmagnetic metals [165]. They are promising candidates for the basic research in magnon caloritronics and for the development of efficient thermoelectric conversion devices.

In order to understand the underlying physics of the SSE in magnetic insulators, the coupling between magnons and phonons was experimentally examined in the transverse geometry, when the thermal gradient is applied in the plane of a magnetic film. A new experimental method based on the Brillouin light scattering technique was developed, which allows for the local measurement of the magnetization and, thus, of the magnon temperature by measuring the temperature-dependent frequency shift of a particular spatially localized magnon mode [166]. Simultaneously, the phonon temperature was monitored using an infrared camera. An unexpectedly close correspondence between the spatial dependences of the exchange magnon and phonon temperatures was revealed [166]. This result indicates that if the transverse spin Seebeck effect is caused by a temperature difference between the magnon and phonon baths, it must be the case that the magnon temperature is spectrally nonuniform, and that the effect is driven by the sparsely populated dipolar region of the magnon spectrum. Afterward, D. Meier et al. attributed the measured voltages in the platinum $(\mathrm{Pt})$ layer in the geometry of the transverse SSE to the LSSE contribution generated by a contact-tip-induced parasitic out-of-plane temperature gradient [167].

Shortly after the discovery of the longitudinal spin Seebeck effect, many investigations were concentrated on the temporal behavior of the LSSE in ferromagnetic-insulator/normal-metal systems. In the first articles on this topic, the representatives of G.A. Melkov's scientific school proposed and proved the crucial role of the bulk-magnon transport in the temporal evolution of the longitudinal spin Seebeck effect [168]. It was revealed that the LSSE is a submicrosecond fast phenomenon [168-170] governed by the thermal-magnon diffusion along the thermal gradient inside a magnetic material. The effective thermal-magnon diffusion length for the YIG/Pt system was estimated to be around $500 \mathrm{~nm}[168,170]$ that was supported by the obtained similar results in a different group [171]. The further investigations detected the LSSE evolution on the nanosecond time scale [172] for relatively thick magnetic films, afterward even on the picosecond [173] and femtosecond [174] time scales for nanometer thicknesses of a magnetic film.

The proposed method of estimation of the magnon propagation length by fitting the transient LSSE response [168] was also applied to the bulk YIG samples covered with a Pt layer [175]. Moreover, the propagation time of thermally diffusing magnons to the YIG/Pt interface and their movement through a spatially varying temperature gradient were taken into account to improve the original model. The propagation length for a bulk sample was estimated in the range of a few micrometers [175], and it shows a strong dependence on the applied magnetic field. 
The improved method was applied recently to the transient behavior of the spin current generated by the LSSE in a set of platinum-coated YIG films of different thicknesses [176]. It was confirmed that that the time evolution of the LSSE is determined by the evolution of the thermal gradient triggering the flux of thermal magnons in a vicinity of the YIG/Pt interface. Moreover, it was suggested that these magnons move ballistically within the YIG film with a constant group velocity, while their number decays exponentially within an effective propagation length. The ballistic flight of the magnons with energies above $20 \mathrm{~K}$ is a result of their almost linear dispersion law similar to that of acoustic phonons. By fitting the timedependent LSSE signal for different film thicknesses varying by almost an order of magnitude, it was found that the effective magnon propagation length of $435 \mathrm{~nm}$ is practically independent of the YIG film thickness [176]. This fact was considered as a strong support of a ballistic transport scenario of quasiacoustic magnons in YIG at room temperature.

In contrast to the presented above local LSSE experiments, nonlocal electric measurements of the thermal-magnon transport in a magnetic insulator result in much larger values of the magnon relaxation length of the order of micrometers [177] even at room temperature. The performed comprehensive investigation of the nonlocal SSE signals in wide distance and temperature ranges reveals different regimes for the nonlocal transport of thermally generated magnons $[178,179]$. The observed discrepancy in the magnon relaxation length for local and nonlocal measurements indicates the necessity of the further magnoncaloritronic investigations for a better understanding of the underlying physics.

\section{Spintronics}

Spintronics, which studies the spin-charge coupling in condensed matter and searches for ways of the application of spin-charge coupling phenomena in electronics [180], is another emergent field of modern magnetism. An explosive growth of interest in spintronics was caused by the discovery of the giant magnetoresistance effect in magnetic valves, which later became the basis of read-head in all magnetic hard drives. The second renewal of the scientific interest in spintronics happened after the experimental confirmation of the spin-transfer-torque (STT) effect $[181,182]$, which is an "inverse" effect to giant or tunnel magnetoresistance. The STT effect allows a manipulation of the magnetization by a dc electric current, making possible, in particular, the excitation of a coherent magnetization precession by a dc current $[181,182]$. This phenomenon was used for the creation of spin-torque oscillators (STOs) - nanoscale microwave auto-oscillators, which became intensively studied in relation to potential applications as compact microwave sources [183], sources of propagating spin waves for purposes of magnonics [184], basic elements for neuromorphic computing [185], etc.

Although there are many different designs of STOs, the basic operation principle of all of them is the same. The spin current, injected into a ferromagnetic layer, exerts STT on the layer magnetization, which, under certain conditions, acts as the "anti-damping" torque. Consequently, if the injected current overcomes a certain threshold, it could completely compensate losses and, eventually, lead to the excitation of self-sustained magnetization oscillations at a microwave frequency. The spin current can be created by the spin filtering of an electric current in the adjacent ferromagnetic layer, spin Hall effect in a heavy metal layer, etc.

G.A. Melkov and representatives of his scientific school also became interested in novel phenomena of STT and started to investigate the microwave magnetization dynamics of various spin-torque oscillators (see Fig. 7). The major breakthrough was made in the understanding of the STO dynamics with a continuous free layer (nanocontact STO, Fig. 7, a). It was theoretically suggested $[105,186]$ and then confirmed by micromagnetic simulations [187] that, under certain conditions, the excited mode in an STO is not a common linear spin-wave mode, but a nonlinear self-localized spin-wave bullet. The appearance of the bullet mode is related to a negative nonlinear shift of the spin-wave frequency in ferromagnetic films magnetized in-plane or at a small angle to the film plane. Due to a negative nonlinear shift, the bullet frequency decreases with increasing its amplitude and goes below the spectrum of propagating spin waves, resulting in the nonpropagating evanescent character of the bullet mode. These researches explained a significant difference of the measured and calculated (within the linear theory) thresholds of STO excitation in preceding experiments. In the following, the self-localized nature of the bullet mode was confirmed in many experiments using the direct imag- 
ing (see, e.g., Ref. [188] for review). Later, peculiarities of the bullet formation in a one-dimensional STO (nanowire spin-Hall oscillators, Fig. 7, c) were investigated [189]. Comparing to the two-dimensional case (free layer in the form of a film), the role of the magnetodipolar interaction was shown to drastically increase, allowing the stabilization of a large, micronsize bullet (in contrast to $100-200 \mathrm{~nm}$ in films).

Another series of important results on STOs was summarized in the review [190]. This work concerns nanopillar STOs (Fig. 7, b) or nanocontact STOs characterized by a positive nonlinear frequency shift (e.g., magnetized out-of-plane). A general analytic approach to the theory of microwave generation in STOs, based on the universal model of an autooscillator, was developed. The model describes the power and frequency of a generated microwave signal as functions of the bias current and the magnetic field and allows one to predict the magnitude and properties of the generation linewidth. It also explains the behavior of an STO under the influence of periodic and stochastic external signals such as the frequency modulation, phase-locking to external signals, mutual phase-locking in an array of STOs, broadening of the generation linewidth near the generation threshold, etc. Being simultaneously simple and general, this model became widely used in studies of STOs, in particular, in the characterization of fabricated STOs, precise determination of the generation threshold, designing of STOs for specific applications, etc. Later, the developed model was generalized to the case of vortex STOs [191].

Significant disadvantages of conventional ferromagnet-based STOs are their rather low operation frequencies $(\sim 1-20 \mathrm{GHz})$ and output microwave powers ( $\sim 1 \mathrm{nW}$ for STOs utilizing the giant magnetoresistance effect and up to $\sim 1 \mu \mathrm{W}$ for STOs based on magnetic tunnel junction structures). To eliminate these disadvantages, a novel method of extraction of the generated signal power from STOs was considered in [192], where the generated power was received as the direct electromagnetic emission of two effective magnetic dipoles. It was shown that this mechanism of signal extraction might have an advantage for sufficiently large arrays $\left(10^{2}-10^{3}\right.$ elements) of coupled high-frequency $(\sim 10-100 \mathrm{GHz})$ STOs. The developed model was later modified and used for terahertz-frequency antiferromagnetic spin Hall oscillators [193]. Another approach to improve the output

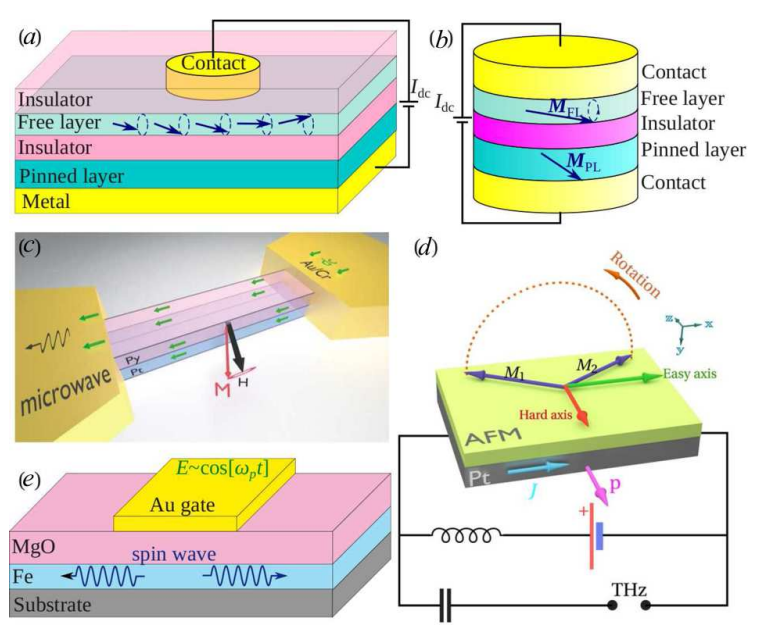

Fig. 7. Sketches of the discussed spintronic devices: nanocontact STO $(a)$, nanopillar STO $(b)$, nanowire spin-Hall oscillator $(c), \operatorname{AFM}$ STO $(d)$, and VCMA-based device $(e)$. Images are adopted from Refs. [189, 190, 206, 212]

signal frequency and power of the generating STO was considered in [194-196]. It was shown that the proposed dual-free-layer STO can provide the doubling of the output signal frequency and power, but the operation of such an STO is strongly hysteretic as a function of the bias dc current. This hysteresis is caused by the dipolar coupling between the free layers; it implies the possibility of application of a strong initial pulse of the bias current (greater than the upper threshold of the stable dynamics) and the subsequent reduction of the bias current to a working point (lying between the low and upper thresholds) corresponding to the required output STO frequency.

In STOs, the dc electric current excites the microwave magnetization dynamics. Simultaneously, an inverse effect, which was called the spin-torque diode effect, is also possible [197]. The spin-torque diode effect is a rectification effect of the input microwave current in a magnetoresistive junction. In this case, the resonance oscillations of the junction resistance can mix with the oscillations of the input microwave current and produce a large enough output dc voltage. The devices based on this effect became called the spin-torque microwave detectors (STMDs). It was demonstrated that STMDs could operate in two distinct regimes, one of which is characterized by a small-angle in-plane magnetization precession (IPregime) [197], while the other one - by a largeangle out-of-plane magnetization precession (OOP- 
regime) [198]. The switching between these regimes occurs under an applied bias magnetic field and/or dc current. In the IP-regime, STMD works as a standard resonance quadratic detector, performance of which is limited by the Johnson-Nyquist or magnetic noise depending on the input microwave power $[199,200]$. In contrast, in the OOP-regime, STMD operates as a threshold detector of low-frequency microwave signals, which might also be interesting for the energy harvesting applications [198]. The theory of STMD operation in both regimes is summarized in $[201,202]$. Also a promising novel type of frequencytunable STMD based on a rapidly-tuned STO has been recently proposed in [203].

The STT effect is present not only in ferromagnetic materials. Antiferromagnets (AFMs) also demonstrates STT [204, 205]. However, the traditional method of the STT-induced damping compensation, used in ferromagnetic STOs, does not work for AFMs. Since AFMs have two magnetic sublattices with opposite magnetizations (or several sublattices with zero net magnetization), the STT, by decreasing the damping in one of the sublattices, increases it in the other sublattice, thus resulting in the zero net effect. The rotation of sublattice magnetizations with frequencies in the $\mathrm{THz}$ range can be induced by a different polarization of the spin current - perpendicularly to the easy plane of AFM. Unfortunately, this rotation does not lead to the appearance of any detectable $\mathrm{THz}$ signal. Three possibilities of a realization of THz AFM-STO were proposed in theoretical works [193, 206, 207]. The first one uses an inhomogeneity of the antiferrmagnetic vector precession in AFM with biaxial anisotropy, like $\mathrm{NiO}$ [206] (Fig. $7, d$ ); the second possibility is based on the application of canted AFM having a small net magnetic moment due to the influence of the DzyaloshinskiiMoriya interaction [193]. In the third one, the generated ac signal is extracted through a variation of the tunneling anisotropic magnetoresistance in a dc-biased antiferromagnetic tunnel junction $\mathrm{Pt} / \mathrm{AFM} / \mathrm{MgO} / \mathrm{Pt}$ [207]. AFM-STOs are promising not only as a compact sources of $\mathrm{THz}$ signals, but also as artificial "neurons" [208] and ultrafast logic elements for the processing of digital signals [209].

The last spintronic effect, intensively investigated by representatives of G.A. Melkov's scientific school, is the voltage-controlled magnetic anisotropy (VCMA). Although VCMA is not often considered as a spintronics effect, in fact, it is. The nature of VCMA is the spin-dependent screening of conduction electrons at the interface of a ferromagnetic metal, and their interaction with localized magnetic moments in a metal. As a result, the application of an electric field to the ferromagnetic metal surface (ferromagnetic metal - dielectric interface) leads to a variation of the surface perpendicular anisotropy of a ferromagnet [210, 211]. Applications of VCMA benefit from the simplicity of a fabrication of nanoscale structures and ultralow power losses in VCMA transducers due to the zero Joule heating. In particular, it could be used in spin-wave processing devices for the excitation, controllable reflection, amplification, and stabilization of the amplitude and phase of propagating spin waves, as was proposed and demonstrated in [212-214]. Interestingly, that the external drive by a microwave voltage via VCMA could be not equivalent to the drive by a microwave magnetic field. For example, features of the VCMA drive allow one to realize the efficient parametric coupling to spin waves of arbitrary wavelengths, including short exchangedominated spin waves. In contrast to the common parallel pumping, the efficiency of which becomes negligible for short spin waves due to vanishing the ellipticity, the VCMA parametric coupling efficiency in the case of in-plane magnetized ferromagnet is proportional to the out-of-plane dynamic magnetization component, which is always nonvanishing [212].

Spintronics effects and devices, described in this section, could find various applications starting from the microwave and $\mathrm{THz}$ signal generation and detection, to the application in spin-wave signal processing and all-magnonic logic circuits.

\section{Magnonic Crystals}

Magnonic crystals (MCs) are artificial magnetic media with periodic variation of their magnetic properties in space. Their name is related to a magnon the quasiparticle associated with a spin wave (SW) a propagating disturbance in the local magnetic order. Similarly to photonic crystals operating with light, the Bragg scattering affects the SW spectrum in such a periodic structure and leads to the formation of band gaps - frequencies, at which the SW propagation is prohibited. MCs use the wave nature of magnons to obtain magnon propagation characteristics that are inaccessible by any other means (see reviews $[155,215-217])$. This allows one to combine one- and

ISSN 2071-0194. Ukr. J. Phys. 2019. Vol. 64, No. 10 
(a)
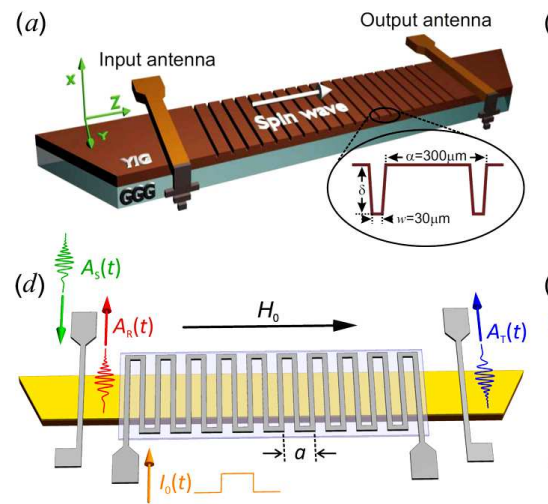

(b)

(e)

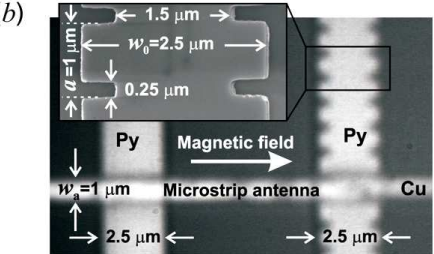

(c)

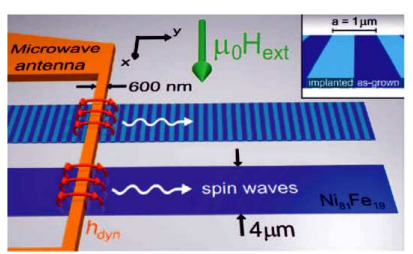

(f)

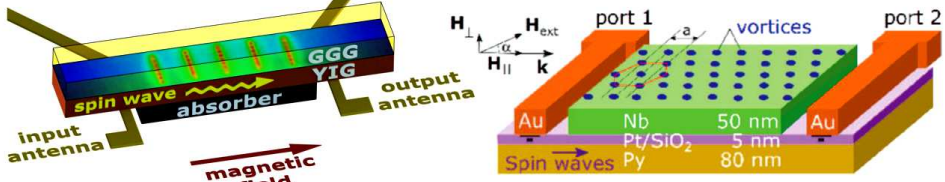

Fig. 8. Sketches and photographs of some magnonic crystals discussed in the article. Images in panels $(a)-(f)$ are adopted from Refs. [162, 223, 228, 231, 234, 237], respectively

two-dimensional magnon waveguidings with the magnon-based data processing [218]. Furthermore, the reconfigurable [217] and dynamic [219] MCs demonstrate a potential for the realization of universal data processing units, which can perform different operations with data on demand [220].

Figure 8 shows the main classes of MCs that have been developed, fabricated, and studied by the representatives of G.A. Melkov's scientific school. Yttrium Iron Garnet $\left(\mathrm{Y}_{3} \mathrm{Fe}_{5} \mathrm{O}_{12}, \mathrm{YIG}\right)$ ferrite films, grown by the liquid phase epitaxy on a Gadolinium Gallium Garnet (GGG) substrate, were chosen as the principal magnetic material for these studies so as to capitalize on YIG's uniquely low magnetic damping $[155,156]$. At the same time, thin metal ferromagnetic films prepared, e.g., from Permalloy $\left(\mathrm{Ni}_{81} \mathrm{Fe}_{19}\right.$, $\mathrm{Py}$ ) were utilized for the fabrication of microstructured MCs.

Different methods of MC fabrication have been developed, tested, and used: (i) periodic variation of the thickness of a YIG-based magnon waveguide [161, 221-227] (Fig. 8, a); (ii) variation of the width of magnon waveguides made of YIG or Py [228-230] (Fig. 8, b); (iii) change of the saturation magnetization of a $\mathrm{Py}$ waveguide by the ion implantation [231, 232] (Fig. 8, c); (iv) spatial modulation of a bias magnetic field via current-carrying wires [219, 233235] (Fig. 8, d); (v) thermally induced variations of the saturation magnetization by the laser-light illumination [162] (Fig. 8,e); (vi) variation of the effective magnetization of a magnetic medium by mechanically induced surface acoustic waves [236], and (vii) twodimensional variations of a local magnetic field by a lattice of superconducting flux-lines [237] (Fig. 8, f).
In-depth preliminary studies of the SW propagation through magnetic nonuniformities and inhomogeneities of different physical nature underpin the developed designs of MCs [232,238-241]. For example, the investigations of the SW transfer through mechanical gaps in longitudinally magnetized YIG film waveguides [238] determined that only slight variations in the YIG film thickness (instead of an approach of "interacting magnetic stripes" separated by air gaps [215,217]) should be employed for the crystal design shown in Fig. 8, a. The extensive research of the SW scattering on magnetic inhomogeneities created by a current conducting wire was done both in the quasistatic [239] and in dynamic modes of operation [240] prior to the fabrication of a currentcontrolled MC shown in Fig. 8d. Different scattering regimes corresponding to the formation of a magnetic well or a magnetic barrier - diffractive scattering and SW tunneling - were analyzed. In Ref. [241], the trapping of a SW packet between two dynamically controlled current conducting wires was studied by means of the time- and space-resolved Brillouin Light Scattering (BLS) spectroscopy and analyzed theoretically. The obtained knowledge enabled us to determine the appropriate value of the applied current required to achieve good band gap rejection efficiency in a current-controlled MC [233].

\subsection{Groove-array-based magnonic crystals}

The geometric structuring of a uniform SW waveguide by the fabrication of an array of grooves on its surface is a simple highly efficient means of MC fabrication [221-223] (see Fig. 8, a). The photolithographic patterning followed by the hot phospho- 
ric acid etching is a reliable tool of forming the required grooves in YIG. The spectra of transmitted SWs in such structures were studied experimentally and theoretically for the backward volume [219, 220] and surface SW modes [223]. A special attention was paid to the study of MC microwave characteristics as functions of the groove depth [221-223]. Rejection frequency bands have been clearly observed, and it has been shown that their depth and the frequency width increase with the groove depth.

The detailed studies of these MCs and their optimization have been performed in Ref. [223]. It has been found that the rejection efficiency for the SW volume mode (wavevector and internal magnetic field are collinear as shown in Fig. 8, a) is dramatically (600 times) higher than that of the surface one (wavevector and the internal field form $90^{\circ}$ angle) [223]. This experimental finding can be related to the protection of magnetostatic surface waves against their backscattering from surface defects [242]. The protection is caused by the chirality of the mode profiles of two counterpropagating waves. This phenomenon is predicted for magnetic films of nanoscale thickness analytically and is confirmed by means of micromagnetic simulation [242].

Due to the predictability and robustness of the grooved MCs, they were utilized in fundamental experiments on the nonlinear SW dynamics [226], caloritronic studies [161], for wavevector-dependent mode selection in a self-exciting positive-feedback SW oscillator $[224,225]$ and for the creation of a magnon transistor [227].

\subsection{Microstructured magnonic crystals}

In order to satisfy the demand of modern signal processing applications for microscopic MCs, a nanoscaled MC was fabricated by engineering periodic variations in the width of a Permalloy waveguide (see Fig. 8, b). The SW propagation through such a structure was measured by means of the microfocused BLS spectroscopy [228]. A SW band gap was clearly observed, and the ability to shift its frequency via the applied bias magnetic field was demonstrated. It was the first experimental realization of the propagation of artificially excited SWs through a microstructured MC.

The transmission characteristics of the Py-based width-modulated crystals were also investigated by numerical simulations [229]. By choosing a step-like or sinusoidal variation of the width, the MC revealed multiple or single band gaps, respectively.

Alternatively, a micromagnetic analysis of the SW propagation in a $\mathrm{MC}$ realized as a $\mathrm{Py} \mathrm{SW}$ waveguide with a spatial periodical variation of its saturation magnetization has been performed [232]. Frequency band gaps were clearly observed in the SW transmission spectra, and their origin is traced back to an overlap of individual band gaps of the fundamental and higher-order SW width modes. This superposition can be controlled by the width of an MC waveguide. Furthermore, the depths of the rejection bands depend strongly on both the level of the magnetization variation and the size of the area with a reduced magnetization: a reduction of the saturation magnetization over a larger area leads to the formation of more pronounced band gaps. In addition, it was shown that the MC could act as a selective filter of SW modes, if specific excitation frequencies are used.

As a proof of the concept, a microstructured Pybased MC was fabricated by the localized ion implantation (see Fig. 8,c) and was investigated in comparison with a reference Py waveguide using the microfocused BLS spectroscopy [231]. The irradiation caused a periodic variation in the saturation magnetization along the waveguide. It has been found that a relatively weak modification of the saturation magnetization by $7 \%$ is sufficient to decrease the SW transmission in the band gaps by a factor of 10 . Since the waveguide consisted of a single topographically unchanged material, SWs with frequencies in the transmission bands were nearly unaffected.

\subsection{Dynamic magnonic crystals}

Magnonic crystals with properties varying on a time scale faster than the SW propagation time through the crystalline structure are named dynamic MCs (DMCs). Disciples of the scientific school of G.A. Melkov have pioneered the field of DMCs, proposed and realized crystal designs, and developed and tested signal processing algorithms. A DMC employing a ferrite waveguide placed in a spatially periodic dynamically controllable magnetic field provided by a current-carrying metallic meander structure (Fig. 8, $d$ ) was fabricated and studied [233]. The current-carrying structure was spaced at a distance of $100 \mu \mathrm{m}$ above the YIG surface in order to avoid the undesirable interaction between SWs and the con- 
ductor. As the lateral variations of the bias magnetic field are almost sinusoidal, the spectrum of the crystal contains only one space component, and a single rejection band appears solely. Its width and depth can be tuned by the applied current [233]. Furthermore, such a crystal can be switched from a full transmission to a full rejection state in a period ten times smaller than the SW relaxation time providing great opportunities to explore new physical effects.

The first experimental realization of the linear time reversal of a propagating wave packet using such a DMC has been reported in Ref. [234]. A brief activation of the DMC by a short current pulse $I_{0}(t)$ whilst a SW packet $A_{\mathrm{S}}(t)$ is inside the results on the generation of a reflected wave packet $A_{\mathrm{R}}(t)$ having an inverted spectrum and a reversed time profile (see Fig. 8, $d$ ). It allows the frequency inversion of monochromatic signals and the time reversal of complex waveforms $[234,235]$. The reversal mechanism is entirely general and applicable to waves of any nature. Therefore, this work is not only significant in the context of the magnetic dynamics. It demonstrates far more generally that the dynamic phenomena in artificial crystals can provide a substitute for the nonlinear mechanisms traditionally used to perform complex spectral transformations such as the time reversal $[39,45,49]$.

Another kind of a DMC, where electric currents control the effective geometry of a magnetic film waveguide for SWs propagating therein, was proposed in Ref. [230]. The device utilizes a static MC prepared as a SW waveguide with periodically varying width. The MC was fabricated from a YIG film, and two conducting wires were attached to the film surface along the waveguide edges. A spatially uniform bias magnetic field is directed across the YIG waveguide. Electric currents applied to the wires induce Oersted fields that reduce the bias magnetic field near the edges. Such created magnetic wells screen the modulated waveguide's edges from SWs and suppress, thus, the MC band gap. By the use of the control currents as logic inputs and the transmitted microwave signal as a logic output, the functionality of this DMC as an AND logic gate has been demonstrated [230]. Advantages of the widthmodulated DMC in comparison to the ones utilizing the meander wiring [233-235] are: the potentially fast performance caused by its small inductance and the possibility for the miniaturization by the use of nanos-

ISSN 2071-0194. Ukr. J. Phys. 2019. Vol. 64, No. 10 tructured width-modulated waveguides made of Py films (Fig. 8, b), as in Ref. [228].

\subsection{Reconfigurable magnonic crystals}

A nanoscale $\mathrm{MC}$ with time-variable characteristics can also be created using the voltage-controlled perpendicular magnetic anisotropy (PMA) in ferromagnetic-dielectric heterostructures [243]. In such a crystal, a periodic array of gate metallic strips is placed on the top of a $\mathrm{MgO} / \mathrm{Co}$ structure in order to apply a periodic electric field and to modify the PMA in a 1.5 -nm-thick Co film. It was numerically shown that the introduction of PMA leads to the formation of band gaps, which can be switched-on and -off within a few tens of nanoseconds. The SW propagation time through the modeled $\mathrm{MC}$ is approximately $0.8 \mathrm{~ns}$ and is, thus, smaller than the on/off switching times. Therefore, this crystal is termed reprogrammable [217,244], rather than dynamic [219]. Nevertheless, the proposed concept can still be used for a realization of DMCs after the optimization of the on/off switching times, SW group velocity, and lengths of the periodic structure. Furthermore, the proposed MC opens a way to low-power magnonic applications.

It has also been demonstrated that a variety of magnetization patterns can be created in a reconfigurable fashion by the laser heating of a magnetic film [162]. A magnon waveguide was produced from a $5 \mu \mathrm{m}$-thick YIG film, which absorbs $40 \%$ of the incident green light. In order to increase the heating efficiency, the YIG film was coated with a black absorber (Fig. 8,e). The formation of band gaps in the SW transmission characteristic was observed for a number of one- and two-dimensional light patterns formed by a spatial light modulator. The positions of the band gaps were tuned by the lattice constant of the MC, while their widths were controlled by the light intensity.

\subsection{Moving magnonic crystals}

Moving MCs represent a special type of dynamically tunable crystals utilizing moving Bragg grates. Apart from the interesting physics shown by such systems [245], they possess a potential for sensors and signal processing applications [246]. A moving MC created via a periodic strain induced by a surface acoustic wave traveling together with a SW along a longitudinally magnetized YIG-film stripe is described 
in Ref. [236]. The experiment was performed using backward volume SWs having a negative group velocity. As a result, the wave scattered from an approaching grating was shifted down in frequency, demonstrating the reverse Doppler effect [236]. As opposed to Ref. [247], the reflection occurs from a crystal lattice, rather than from a single reflecting surface. Thus, the wavenumber of the scattered SW is strictly determined by a momentum conservation law. Due to this fact, the frequency-shifted wave appears as a single narrow peak in the transmission characteristic of a MC.

Recently, a novel class of moving MCs utilizing a two-dimensional lattice of magnetic flux quanta (fluxons) in ferromagnet/superconductor $\mathrm{Py} / \mathrm{Nb}$ bilayers has been established [237]. Each fluxon represents a whirl of the electric supercurrent shielding the superconductor from the magnetic field emanating from and attaining a maximum at the vortex core. Being able to scatter SWs [248,249], this vortex lattice constitutes a tunable Bragg grating for SWs externally excited in a Py layer (see Fig. 8, f), resulting in characteristic dips in the SW transmission. As the distance between vortices is inversely proportional to the square root of the magnetic field value, this allows a tuning of the transmission bands and gaps in the magnon spectrum on demand. Furthermore, Doppler shifts in the frequency spectra of SWs scattered on a flux lattice moving under the action of a transport electric current in the superconductor have been observed. This suggests magnonic devices, where the fluxonic system allows the externally tunable fast control of magnons by ultra-low electric currents in the superconductor.

\subsection{Storage-recovery phenomenon and nonlinear spin waves in magnonic crystals}

Investigations of parametric interactions between SWs and the electromagnetic pumping in MCs have delivered the extremely interesting insight into nonlinear wave physics. In such systems, parametrically coupled waves propagating in opposite directions are simultaneously coupled by the Bragg reflection law. As a result, the parametric instability in MCs exhibits a strongly phase-dependent behavior [226]. Being interesting by its fundamental physics, this interaction can be used to trap the SW energy and, thus, may find applications in the storage of microwave signals.

It has been shown that, unlike the conventional scheme used in photonics, this trapping occurs not due to the deceleration of the incident wave when it enters the periodic structure [250], but due to the excitation of quasinormal modes of an artificial crystal [226]. The first quasinormal mode, whose eigenfrequency coincides with the minima of the SW group velocity at the edges of the MCs band gaps, has the longest lifetime among all other modes and, thus, conserves the signal energy and phase information for a long time after the propagating wave has left the MC. Such a "trapped" SW signal can subsequently be restored to the traveling form by means of the doublefrequency parametric amplification. The restoration and, thus, the storage occurs only at the edges of the MC band gap. The appearance time and the duration of the restored signal are determined by the pumping power. The restoration mechanism is based on a competitive nonlinear amplification process developed in Refs. [50-52, 251-253] in the case of featureless thin magnetic films. The main difference in the case of a $\mathrm{MC}$ is that the Bragg reflection condition exerts a strong influence on the parametric interaction. This leads to a pronounced dependence of the amplitude of the restored signal on the relative phase of the input and pumping waves.

In general, the development of nonlinear processes such as the frequency multiplication, rectification, parametric amplification/generation, parametric wave front reversal, etc., can be strongly modified by the artificial structuring of a magnetic material. For example, the discretization of the magnon spectrum in a two-dimensional array of cylindrical Py nanodots removes the frequency degeneracy between the externally excited mode (spatially quasiuniform ferromagnetic resonance (FMR) mode) of a nanoparticle and the higher magnon modes with the lowest magnetic damping and reduces, thus, the strength of multimagnon relaxation processes [254-256]. This significantly increases the amplitude and lifetime (up to 3 orders of magnitude) of the FMR mode and intensifies all nonlinear processes with its participation.

\section{Spin-Wave Processing of Binary Data}

One of the main attractive features of magnonics lies in the benefits provided by the wave nature of magnons for the data processing and computa-

ISSN 2071-0194. Ukr. J. Phys. 2019. Vol. 64, No. 10 

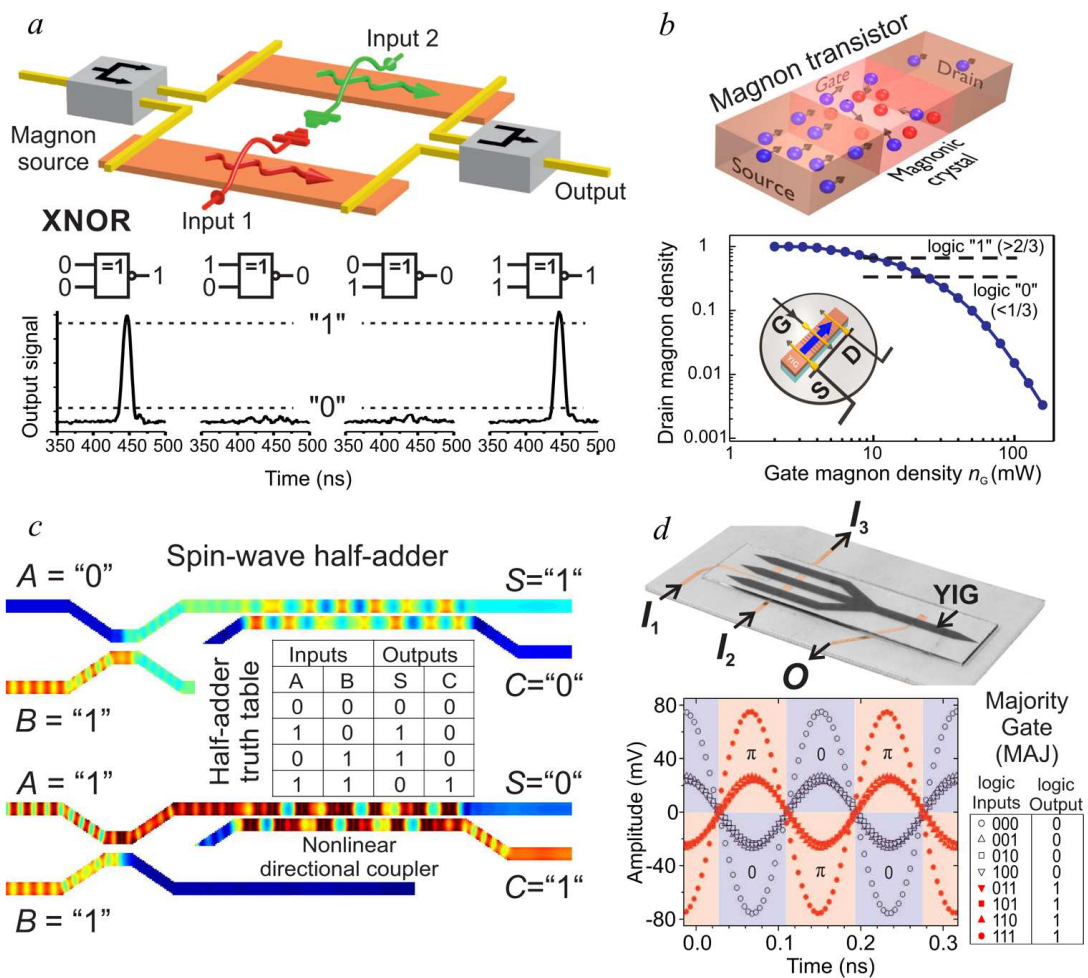

Fig. 9. Spin-wave XNOR logic gate [264, 265] (a). The gate is based on a Mach-Zehnder interferometer with electric currentcontrolled phase shifters embedded in the two magnon conduits. The bottom panel shows the output pulsed spin-wave signals measured for different combinations of the input dc signals applied to the phase shifters. Schematic of the operational principle of a magnon transistor [227]: The source-to-drain magnon current (shown with blue spheres) is nonlinearly scattered by gate magnons (red spheres) injected into the gate region. In the bottom panel, the measured drain magnon density is presented as a function of the gate magnon density $(b)$. First integrated magnonic circuit: All-magnon half-adder based on the usage of two spin-wave directional couplers, one operating in a linear regime, and another coupler in a nonlinear one [267]. Logic data are coded into the spin-wave amplitude (shown with colours). Two examples of different logic inputs are shown (c). Spin-wave majority gate. Upper panel: photograph of the majority gate prototype under testing in Ref. [274]. Spin waves are excited with microstrip antennas $\left(\mathrm{I}_{1}, \mathrm{I}_{2}\right.$, and $\mathrm{I}_{3}$ ) in the input YIG waveguides (width $1.5 \mathrm{~mm}$ ) and propagate through the spin-wave combiner into the output waveguide for the detection with the output antenna (O). Lower panel: Output signal observed with an oscilloscope for different phases of the input signals. The dependence of the output phase on the majority of the input phases corresponds to the truth table $(d)$

tion. In the past, the application area of spin waves was mostly related to the analog signal processing in the microwave frequency range. In those applications, microwave filters, delay lines, phase conjugators, power limiters, and amplifiers are just a few examples [155, 257, 258]. Nowadays, new technologies allowing, e.g., the fabrication of nanometer-sized structures or the operation in the $\mathrm{THz}$ frequency range give, in combination with novel physical phenomena, a new impetus to this field and make the above-discussed advantages accessible for both analog and digital data signal processing. Moreover, magnons also possess the potential to be used in the im- plementation of alternate computing concepts such as factorization units [259], artificial neural networks [260], and the projecting of optical computing concepts onto the nanometer scale [261]. In the following section, the basic ideas behind the standard Boolean logic operations with digital binary data, which are currently the subject of intense theoretical and experimental studies [218], are addressed.

\subsection{Original concepts of the spin-wave logic}

The idea of coding the binary data into a spin-wave amplitude was first stated by Hertel et al. in 2004 [262]. Micromagnetic simulations revealed that mag- 
netostatic spin waves change their phase, as they pass through domain walls. It was suggested to split spin waves in different branches of a ring (spin-wave interferometer). After being merged in the output, their interference depends on the presence of domain walls in the branches. In such a way, a controlled manipulation of phases of spin waves was proposed to be utilized for the realization of spin-wave logical operations. The first experimental studies of the spin-wave logic were reported by Kostylev et al. in [263]. It was proposed that a Mach-Zehnder spin-wave interferometer equipped with current-controlled phase shifters embedded in the interferometer arms can be used to construct logic gates. Following this idea, Schneider et al. realized a proof-of-principle XNOR logic gate shown in Fig. 9, $a[264,265]$. The currents applied to Input 1 and Input 2 represent logic inputs: The current-on state, which results in $\pi$-phase shift of a spin-wave in the interferometer arm, corresponds to logic "1"; the current-off state corresponds to logic " 0 ". The logic output is defined by the interference of microwave currents induced by the spin waves in the output microstrip antennas: A low amplitude (destructive interference) corresponds to logic " 0 ", and a high amplitude (constructive interference) defines logic " 1 ". The change in the magnitude of the output pulsed signal for different combinations of the logic inputs (see Fig. 9, a) corresponds to the XNOR logic functionality. It was also shown that an electric current can create a magnetic barrier reducing or even stopping the spin-wave transmission. Using this effect, a universal NAND logic gate was demonstrated [264]. Lee at al. [266] has proposed an alternative design of a nano-sized logic gate. In that device, the spin-wave phases are controlled by means of an electric current flowing through a vertical wire placed between the arms of an interferometer. This current defines the logic input, while the amplitude of the output spin-wave after the interference defines the logic output. The ability to create NOT, NOR, and NAND logic gates was demonstrated using numerical simulations.

\subsection{Magnon transistor}

The drawback of the discussed logic gates consists in that it is impossible to combine two logic gates without additional magnonto-current and current-to-magnon converters. This fact stimulated the search by the representatives of G.A. Melkov's scientific school for the means to control a magnon current by another magnon current. It has been demonstrated that such control is possible due to nonlinear magnonmagnon scattering, and a magnon transistor was realized [227] - see Fig. 9, b. In this three-terminal device, the density of the magnon current flowing from the Source to the Drain (see blue spheres in the figure) is controlled by the magnons injected into the Gate of the transistor (red spheres). A magnonic crystal in the form of an array of surface grooves [221] is used to increase the density of the gate magnons and, consequently, to enhance the efficiency of the nonlinear four-magnon scattering process used to suppress the Source-to-Drain magnon current. It was shown that the Source-to-Drain current can be decreased by up to three orders of magnitude (see the bottom panel in Fig. 9, b). The potentialities for the miniaturization of this transistor, realization of an integrated magnonic circuit (using the example of an XOR logic gate), increase of its operation speed, and decrease in the energy consumption were discussed in [227].

\subsection{Toward an integrated magnonic circuit}

Although the operational characteristics of a magnon transistor in its proof-of-principle form [227] did not overcome those of semiconductor devices, it played an important role for a future all-magnon technology, in which information will be carried and processed solely by magnons. The main advantage of the allmagnon approach is that it does not require convertors between magnons and charge currents in each unit. The naturally strong nonlinearity of spin waves is assumed to be used to perform operations with data. A first integrated magnonic circuit was recently demonstrated in Ref.[267] on the example of a halfadder built from two logic gates by means of micromagnetic simulations.

The main building block of the half-adder is a spinwave directional coupler, which was first investigated by Sadovnikov et al. in Ref. [268] on the macroscale and, further, numerically by Wang et al. on the nanoscale [269]. This device consists of two spin-wave waveguides separated by a little gap in order to provide their dipolar coupling. As a result, a spin-wave excited in one waveguide will periodically pump all its energy to the neighboring waveguide after the propagation of a certain distance and back. It was shown numerically [269] and very recently experimentally on the nanoscale [270] that the information coded

ISSN 2071-0194. Ukr. J. Phys. 2019. Vol. 64, No. 10 
into the spin-wave amplitude can be guided toward one of the two outputs of a directional coupler depending on the signal frequency and magnitude and on the magnetic field. By controlling these degrees of freedom, the multifunctionality and reconfigurability of a directional coupler are achieved. The operation of the directional coupler as a microwave filter, frequency separator, multiplexer, fan-out, AND, and XOR logic gates was demonstrated [267,269]. Moreover, the magnonic half-adder [267] has a strikingly simple design consisting of two directional couplers only - see Fig. 3, $c$ : The first one works in a linear regime and acts as a symmetric power splitter for each of the two inputs. The second coupler operates in a nonlinear regime and simultaneously performs XOR and AND logic operations. It was shown that the proposed magnonic half-adder in combination with a spin-wave amplifier can elegantly replace the needed 14 transistors in the modern complementary metaloxide-semiconductor (CMOS) technology.

\subsection{Spin-wave majority gate}

In the approaches discussed above, logic data was coded into a spin-wave amplitude (a certain spinwave amplitude defines logic "1", zero amplitude correspond to logic "0"). Alternatively, Khitun et al. proposed [271] to use the spin-wave phase for digitizing information instead of the amplitude. A wave with some chosen phase $\phi_{0}$ corresponds to a logic "0" while a logic " 1 " is represented by a wave with phase $\phi_{0}+\pi$. Such an approach allows the trivial embedding of a NOT logic element (which requires two transistors in the modern CMOS technology) in magnonic circuits by simply changing the position of a read-out device by a $\lambda / 2$ distance. Moreover, it opens the access to the realization of a majority gate in the form of a multiinput spin-wave combiner [271]. The spinwave majority gate consists of three input waveguides (generally speaking, of any odd number of inputs larger than one), in which spin waves are excited and merged afterward in a spin-wave combiner. Thus, the majority logic function can be realized due to a simple interference between the three input waves - see the truth table on the right in Fig. 9, $d$. The majority gate can perform not only the majority operation, but also AND and OR operations (as well as NAND and NOR operations, if to use a half-wavelength long inverter), if one of its inputs is used as a control input [272].

ISSN 2071-0194. Ukr. J. Phys. 2019. Vol. 64, No. 10
The first fully functioning design of the majority gate employing a spin-wave combiner was demonstrated using micromagnetic simulations by Klingler et al. in Ref. [272]. The improved design of the majority gate operating with isotropic forward volume magnetostatic spin waves in an out-of-plane magnetized spin-wave majority gate was reported in [273]. In both designs, the phases of the output spinwave clearly satisfied the majority function proving the functionality of the gate. An experimental prototype of a majority gate utilizing a macroscopic YIG structure was shown by Fischer et al. [274]. The respective device comprises three input lines, as well as one output line and has been structured from a YIG film - see the top panel in Fig. 9, $d$. In this geometry, inductively excited spin waves propagate coherently along the three input waveguides and are eventually superimposed, when they leave the spin-wave combiner, at which point the input lines merge. The resultant spin-wave induces an electrical signal in a copper stripline at the output, which is directly mapped by a fast oscilloscope [274] - see Fig. 9, $d$. The phase of the output spin-wave corresponds to the majority phase of the input lines, according to the truth table of a majority gate.

Despite recent advances, the field of spin-wave logic has many challenges remaining both in physical and technological aspects. In particular, low-energy means of the localized magnon excitation, manipulation, and detection on a scale of $10 \mathrm{~nm}$, operations with spin waves of nm-wavelengths and $\mathrm{THz}$ frequencies and the development of efficient spin-wave amplifiers belong to the most actual tasks of the modern magnonics. Nevertheless, the fast growth of the field of beyong-CMOS data processing, the recent breakthrough in the miniaturization of lateral sizes of magnonic structures down to $50 \mathrm{~nm}$ [275], the decrease in wavelengths down to the same value [276], as well as the progress in the understanding and usage of nonlinear spin-wave physics in nano-sized magnonic circuits $[267,270]$ look very promising. The field of the spin-wave logic is therefore an active, emergent field that could deliver a number of breakthrough developments toward applications in data processing in the middle-term future.

\section{Conclusions}

We have reviewed several important directions of the development of modern microwave magnetism and 
superconductivity. Although the studies of superconductivity and microwave magnetism started many decades ago, these fields are developed in many modern relevant trends, which concern both with new fundamental phenomena, as well as with various promising applications in modern technique. Ferromagnetic materials become a nice test bed for the investigation of quantum cooperative phenomena and various nonlinear and chaotic dynamics, as they demonstrate the Bose-Einstein condensation of magnons at room temperature, and many different parametric and nonlinear processes at a moderate drive power. The presented results on HTS resonators and magnonic crystals are directly applicable to the development of microwave devices such as pass-band microwave filters, delay lines, signal sources and detectors including devices that utilize the Josephson effects, and narrow-frequency amplifiers. Important and promising results are achieved in different approaches of the spin-wave computing and binary logic, although many challenges in physics and the technological aspect should be resolved in future. The recent progress in the development of high-temperature superconductors and new magnetic materials, as well as in the high-quality patterning of nanoscale magnetic structures, opens new horizons of possible practical applications and outlines new areas for further researches.

The authors appreciate deeply the opportunities for the joint work with Gennadii A. Melkov and acknowledge him for the scientific guidance, fruitfull discussions, and the outstanding personal contribution to many scientific results described in this review. We acknowledge gratefully the financial support from the Deutsche Forschungsgemeinschaft (DFG projects B01 and B04 of the Research Unit TRR 173 "Spin $+X$ " and project INST 248/178-1), the European Future and Emerging Technologies (EU-FET) program, the European Research Council (ERC Starting Grant 678309 MagnonCircuits and Advanced Grant 694709 SuperMagnonics), the State Fund for Fundamental Research of Ukraine (SFFR), the Deutsche Akademische Austauschdienst (DAAD), the Graduate School Material Science in Mainz (MAINZ), the Alexander von Humboldt Foundation, the $\mathrm{Na}$ tional Science Foundation (NSF) of the USA, the Defense Advanced Research Projects Agency (DARPA), the US Civilian Research and Development Foundation (CRDF), the Center for NanoFerroic De- vices (CNFD) and Nano-electronics Research Initiative (NRI), the Ministry of Education and Science of Ukraine (grants Nos. 18BF052-01M and 19BF05201), the National Academy of Sciences of Ukraine (grant No. 1F). The publication contains the results of studies conducted by President's of Ukraine grant for competitive projects (F 84). We acknowledge I.I. Syvorotka (Scientific Research Company "Electron-Carat", Lviv, Ukraine) for supplying us with YIG films.

1. H. Kamerlingh Onnes. Further experiments with liquid helium. D. On the change of the electrical resistance of pure metals at very low temperatures, etc. V. The disappearance of the resistance of mercury. Commun. Phys. Lab. Univ. Leiden 122b, 124 (1911); reprinted in Proc. K. Ned. Akad. Wet. 14, 113 (1911); see also H. Kamerlingh Onnes. Commun. Phys. Lab. Univ. Leiden. Suppl. 29 (1911).

2. J.G. Bednorz, K.A. Müller. Possible high- $T_{\mathrm{C}}$ superconductivity in the $\mathrm{Ba}-\mathrm{La}-\mathrm{Cu}-\mathrm{O}$ system. Z. Phys. B 64, 189 (1986).

3. A.P. Drozdov, P.P. Kong, V.S. Minkov, S.P. Besedin, M.A. Kuzovnikov, S. Mozaffari, L. Balicas, F.F. Balakirev, D.E. Graf, V.B. Prakapenka, E. Greenberg, D.A. Knyazev, M. Tkacz, M.I. Eremets. Superconductivity at $250 \mathrm{~K}$ in lanthanum hydride under high pressures. Nature 569, 528 (2019).

4. L. Landau, E. Lifshitz. On the theory of the dispersion of magnetic permeability in ferromagnetic bodies. Phys. Z. Sowjetunion 8, 153 (1935).

5. E. Zavoisky. Spin-magnetic resonance in paramagnetics. J. Phys. USSR 9, 245 (1945).

6. J.H.E. Griffiths. Anomalous high-frequency resistance of ferromagnetic metals. Nature 158, 670 (1946).

7. G.A. Melkov, I.L. Zablotskii. Nonlinear impedance of high-temperature superconductor films. Ukr. J. Phys. 35, 921 (1990) (in Russian).

8. G.A. Melkov, I.L. Zablotskii. Surface resistance of singlecrystal $\mathrm{Y}-\mathrm{Ba}-\mathrm{Cu}-\mathrm{O}$ films in a magnetic field. Ukr. J. Phys. 38, 420 (1993) (in Russian).

9. G.A. Melkov, A.L. Kasatkin, V.Yu. Malyshev. The surface impedance of epitaxial HTSC films in mixed-state. Low Temp. Phys. 20, 868 (1994) (in Russian).

10. G.A. Melkov, V.Yu. Malyshev, A.V. Bagada. Microwave impedance of epitaxial high-temperature superconductor films. Low Temp. Phys. 21, 1192 (1995) (in Russian).

11. G.A. Melkov, V.Y. Malyshev. The microwave model of HTS epitaxial films. Czech. J. Phys. 46, 1007 (1996).

12. G.A. Melkov, E.A. Pashitskii. Anomalous behavior of the microwave surface resistance of the high- $T_{C}$ superconductors as a result of the "multi-petal" gap structure in anisotropic s-wave Cooper pairing Czech. J. Phys. 46, 971 (1996).

ISSN 2071-0194. Ukr. J. Phys. 2019. Vol. 64, No. 10 
13. G.A. Melkov, V.Yu. Malyshev, S.K. Korsak. Nonlinear microwave properties of epitaxial HTS films. Low Temp. Phys. 23, 782 (1997).

14. V. Shaternik, M. Belogolovskii, T. Prikhna, A. Shapovalov, O. Prokopenko, D. Jabko, O. Kudrja, O. Suvorov, V. Noskov, Universal character of tunnel conductivity of metalinsulator-metal heterostructures with nanosized oxide barriers. Phys. Procedia 36, 94 (2012).

15. V.M. Pan, V.S. Flis, O.P. Karasevska, V.I. Matsui, I.I. Peshko, V.L. Svetchnikov, M. Lorenz, A.N. Ivanyuta, G.A. Melkov, E.A. Pashitskii, H.W. Zandbergen. Effect of growth-induced linear defects on high frequency properties of pulse-laser deposited $\mathrm{YBa}_{2} \mathrm{Cu}_{3} \mathrm{O}_{7-\delta}$ films. J. Supercond. 14, 105 (2001).

16. V.M. Pan, A.L. Kasatkin, V.A. Komashko, C.G. Tretiatchenko, O.M. Ivanyuta, G.A. Melkov. Two-peak temperature dependence of microwave surface impedance of single-crystalline YBCO thin films: role of pairing symmetry and defect structure. J. Supercond. Nov. Magn. 19, 561 (2006).

17. V.M. Pan, A.A. Kalenyuk, A.L. Kasatkin, O.M. Ivanyuta, G.A. Melkov. Microwave response of perfect $\mathrm{YBa}_{2} \mathrm{Cu}_{3} \mathrm{O}_{7-x}$ thin films deposited on $\mathrm{CeO}_{2}$-buffered saphire: A probe for pairing symmetry. J. Supercond. Nov. Magn. 20, 59 (2007).

18. G.A. Melkov, Yu.V. Egorov. Swihart waves and surface plasmons in a parallel-plate superconducting transmission line. Low Temp. Phys. 26, 108 (2000).

19. G.A. Melkov, Y.V. Egorov, O.M. Ivanyuta, V.Y. Malyshev, H.K. Zeng, Kh. Wu, J.Y. Juang. HTS surface wave resonators. J. Supercond. 13, 95 (2000).

20. G.A. Melkov, O.M. Ivanyuta, O.V. Prokopenko, V.M. Raksha, A.M. Klushin, M. Siegel. Embedding of Josephson junctions in the surface wave resonator in the Ka-band. In: Proceedings of the Fourth International Kharkov Symposium 'Physics and Engineering of Millimeter and Sub-Millimeter Waves' Symposium (MSMW'2001, 4-9 June 2001, Kharkov, Ukraine) 1, 363 (2001).

21. A.M. Klushin, G.A. Melkov, O.M. Ivanyuta, K. Numssen, M. Siegel. Irradiation of multi-junction Josephson arrays embedded in a surface wave resonator. Phys. C 372-376, 305 (2002).

22. G.A. Melkov, Y.V. Egorov, A.N. Ivanyuta, A.M. Klushin, M. Siegel, R. Semerad. Josephson generation of linear array of Josephson junctions in surface wave resonator. Izvestiya VUZ. Radioelektronika 45, 38 (2002) (in Russian).

23. O.M. Ivanyuta, O.V. Prokopenko, V.M. Raksha, A.M. Klushin. Microwave detection using Josephson junction arrays integrated in a resonator. Phys. Status Solidi C 2, 1688 (2005).

24. O.M. Ivanyuta, O.V. Prokopenko, Ya.I. Kishenko, V.M. Raksha, A.M. Klushin. The effect of the external magnetic field on the current-voltage characteristic of HTS Josephson junction arrays. J. Low Temp. Phys. 139, 97 (2005).
25. G.A. Melkov, O.M. Ivanyuta, O.V. Prokopenko, V.M. Raksha. Microwave properties of the surface wave resonator in the below-cutoff waveguide. In: Proceedings of the International Conference "Modern Problems of Radio Engineering, Telecommunications and Computer Science" (TCSET, 18-23 February 2002, Lviv-Slavsko, Ukraine), 75 (2002).

26. G.A. Melkov, A.V. Prokopenko, V.N. Raksha. Rarefaction of the natural oscillation spectrum of a surface wave resonator. Radioelectronics and Communications Systems 47, 20 (2004).

27. G.A. Melkov, A.M. Klushin, O.D. Poustylnik, O.V. Prokopenko, V.M. Raksha. Irradiation of HTS Josephson junctions with the surface wave resonator. In: Proceedings of the The Fifth International Kharkov Symposium on Physics and Engineering of Microwaves, Millimeter, and Submillimeter Waves (MSMW'2004, 21-26 June 2004, Kharkov, Ukraine) 2, 128 (2004).

28. G.A. Melkov, O.V. Prokopenko, V.M. Raksha. Microwave HTS filter based on coupled surface wave resonators. In: Proceedings of the 2007 International Kharkov Symposium Physics and Engineering of Millimeter and Sub-Millimeter Waves (MSMW'2007, 25-30 June 2007, Kharkov, Ukraine) 1, 401 (2007).

29. M. Lorenz, V.M. Pan, V.F. Tarasov, O.M. Ivanyuta, G.A. Melkov, V.V. Vysotskii, V.F. Taborov, I.V. Korotash, C.G. Tretiatchenko. Band-pass filter for $1.8 \mathrm{GHz}$ frequency range using double-sided $\mathrm{YBCO} / \mathrm{Au}$ films on $\mathrm{CeO}_{2}$-buffered sapphire. J. Supercond. 14, 115 (2001).

30. G.A. Melkov, S.V. Sholom. Parametric excitation of spin waves by electromagnetic pumping in the structure of magnetic film - HTS film. Abstracts of the Vth AllSoviet Union School on Spin-Wave Microwave Electronics (Zvenigorod, USSR), 35 (1991) (in Russian).

31. G.A. Melkov, Y.V. Egorov. A possibility of parametric amplification of microwave signals in HTS films. Izvestiya VUZ. Radioelektronika 8, 52 (1997) (in Russian).

32. G.A. Melkov, I.V. Krutsenko. Mechanisms that limit the amplitudes of parametrically excited spin waves in ferrites. J. Exp. Theor. Phys. 45, 295 (1977).

33. I.V. Krutsenko, V.S. L'vov, G.A. Melkov. Spectral density of parametrically excited waves. J. Exp. Theor. Phys. 48, 561 (1978).

34. A.S. Bakai, I.V. Krutsenko, G.A. Melkov, G.G. Sergeeva. Investigation of distributions of spin waves excited parametrically by parallel pumping of spin-wave instability in ferrites. J. Exp. Theor. Phys. 49, 118 (1979).

35. A.V. Lavrinenko, V.S. L'vov, G.A. Melkov, V.B. Cherepanov. "Kinetic" instability of a strongly nonequilibrium system of spin waves and tunable radiation of a ferrite. J. Exp. Theor. Phys. 54, 542 (1981).

36. G.A. Melkov, A.Yu. Taranenko. Nonlinear emission from a ferrite in ferromagnetic resonance. J. Exp. Theor. Phys. 64, 592 (1986).

37. V.S. Lutovinov, G.A. Melkov, A.Yu. Taranenko, V.B. Cherepanov. Kinetic instability of first order spin waves in ferrite. J. Exp. Theor. Phys. 68, 432 (1989). 
38. G.A. Melkov, V.L. Safonov, A.Y. Taranenko, S.V. Sholom. Kinetic instability and bose condensation of nonequilibrium magnons. J. Magn. Magn. Mater. 132, 180 (1994).

39. G.A. Melkov, A.A. Serga, V.S. Tiberkevich, A.N. Oliynyk, A.N. Slavin. Wave front reversal of a dipolar spinwave pulse in a nonstationary three-wave parametric interaction. Phys. Rev. Lett. 84, 3438 (2000).

40. G.A. Melkov, V.I. Vasyuchka, Yu.V. Kobljanskyj, A.N. Slavin. Wave-front reversal in a medium with inhomogeneities and an anisotropic wave spectrum. Phys. Rev. B 70, 224407 (2004).

41. G.A. Melkov, V.I. Vasyuchka, A.V. Chumak, A.N. Slavin. Double-wave-front reversal of dipole-exchange spin waves in yttrium-iron garnet films. J. Appl. Phys. 98, 074908 (2005).

42. G.A. Melkov, V.I. Vasyuchka, A.V. Chumak, V.S. Tiberkevich, A.N. Slavin. Wave front reversal of nonreciprocal surface dipolar spin waves. J. Appl. Phys. 99, 08P513 (2006).

43. G.A. Melkov, V.I. Vasyuchka, V.V. Lazovskiy, V.S. Tiberkevich, A.N. Slavin. Wave front reversal with frequency conversion in a nonreciprocal medium. Appl. Phys. Lett. 89, 252510 (2006).

44. B.Ya. Zel'dovich, R.F. Pilipetskii, V.V. Shkunov. Principles of Phase Conjugation (Springer, 1985).

45. G.A. Melkov, A.A. Serga, A.N. Slavin, V.S. Tiberkevich, A.N. Oleinik, A.V. Bagada. Parametric interaction of magnetostatic waves with a nonstationary local pump. J. Exp. Theor. Phys. 89, 1189 (1999).

46. A.A. Serga, B. Hillebrands, S.O. Demokritov, A.N. Slavin, P. Wierzbicki, V. Vasyuchka, O. Dzyapko, A. Chumak. Parametric generation of forward and phaseconjugated spin-wave bullets in magnetic films. Phys. Rev. Lett. 94, 167202 (2005).

47. Yu.V. Kobljanskyj, V.S. Tiberkevich, A.V. Chumak, V.I. Vasyuchka, G.A. Melkov, A.N. Slavin. Methods of relaxation reversal for spin waves and oscillations. J. Magn. Magn. Mater. 272-276, 991 (2004).

48. Yu.V. Kobljanskyj, G.A. Melkov, V.S. Tiberkevich, V.I. Vasyuchka, A.N. Slavin. Microwave signal processing using dipole-exchange spin waves. J. Appl. Phys. 93, 8594 (2003).

49. V.I. Vasyuchka, G.A. Melkov, A.N. Slavin, A.V. Chumak, V.A. Moiseienko, B. Hillebrands. nonresonant wave front reversal of spin waves used for microwave signal processing. J. Phys. D: Appl. Phys. 43, 325001 (2010).

50. G.A. Melkov, Yu.V. Kobljanskyj, A.A. Serga, V.S. Tiberkevich, A.N. Slavin. Reversal of momentum relaxation. Phys. Rev. Lett. 86, 4918 (2001).

51. A.V. Chumak, A.A. Serga, G.A. Melkov, V. Tiberkevich, A.N. Slavin, B. Hillebrands. Parametrically-stimulated recovery of a microwave signal using standing spin-wave modes of a magnetic film. Phys. Rev. B 79, 014405 (2009).

52. S. Schäfer, A.V. Chumak, A.A. Serga, G.A. Melkov, B. Hillebrands. Microwave spectral analysis by means of nonresonant parametric recovery of spin-wave signals in a thin magnetic film. Appl. Phys. Lett. 92, 162514 (2008).

53. V.I. Vasyuchka, A.A. Serga, C.W. Sandweg, D.V. Slobodianiuk, G.A. Melkov, B. Hillebrands. Explosive electromagnetic radiation by the relaxation of a multimode magnon system. Phys. Rev. Lett. 111, 187206 (2013).

54. Yu.V. Kobljanskyj, G.A. Melkov, A.A. Serga, V.S. Tiberkevich, A.N. Slavin. Effective microwave ferrite convolver using a dielectric resonator. Appl. Phys. Lett. 81, 1645 (2002).

55. V.I. Vasyuchka, G.A. Melkov, V.A. Moiseienko, A.V. Prokopenko, A.N. Slavin. Correlation receiver of below-noise pulsed signals based on parametric interactions of spin waves in magnetic films. J. Magn. Magn. Mater. 321, 3498 (2009).

56. G.A. Melkov, D.V. Slobodianiuk, V.S. Tiberkevich, G. De Loubens, O. Klein, A.N. Slavin. Nonlinear ferromagnetic resonance in nanostructures having discrete spectrum of spin-wave modes. IEEE Magn. Lett. 4, 4000504 (2013).

57. G.A. Melkov, D.V. Slobodianiuk. A strongly nonequilibrium state in magnetic nanodots at high pumping levels. Ukr. J. Phys. 58, 189 (2013).

58. D.V. Slobodianiuk, O.V. Prokopenko, G.A. Melkov. Eigenmodes of two-dimensional conservative droplet soliton. J. Magn. Magn. Mater. 439, 144 (2017).

59. D. Slobodianiuk, G. Melkov, K. Schultheiss, H. Schultheiss, R. Verba. Nonlinear ferromagnetic resonance in the presence of 3-magnon scattering in magnetic nanostructures. IEEE Magn. Lett. 10, 6103405 (2019).

60. A.M. Kosevich, B.A. Ivanov, A.S. Kovalev. Magnetic solitons. Phys. Rep. 194, 117 (1990).

61. R.P. Cowburn, D.K. Koltsov, A.O. Adeyeye, M.E. Welland. Single-domain circular nanomagnets. Phys. Rev. Lett. 83, 1042 (1999).

62. T. Shinjo, T. Okuno, R. Hassdorf, K. Shigeto, T. Ono. Magnetic vortex core observation in circular dots of permalloy. Science 289, 930 (2000).

63. U.K. Rossler, A.N. Bogdanov, C. Pfleiderer. Spontaneous skyrmion ground states in magnetic metals. Nature Lett. 442, 797 (2006).

64. S. Muhlbauer, B. Binz, F. Jonietz, C. Pfleiderer, A. Rosch, A. Neubauer, R. Georgii, P. Boni. Skyrmion lattice in a chiral magnet. Science 323, 915 (2009).

65. A.G. Gurevich, G.A. Melkov. Magnetization Oscillations and Waves (CRC, 1996).

66. B.A. Kalinikos, A.N. Slavin. Theory of dipole-exchange spin-wave spectrum for ferromagnetic films with mixed exchange boundary conditions. J. Phys. C-Solid State Phys. 19, 7013 (1986).

67. A.N. Slavin, B.A. Kalinikos, N.G. Kovshikov. Chapter 9 in P.E. Wigen (Ed.) Nonlinear Phenomena and Chaos in Magnetic Materials (World Scientific, 1994).

68. N.N. Akhmediev, A. Ankiewicz. Solitons, Nonlinear Pulses and Beams (Chapman and Hall, 1997).

ISSN 2071-0194. Ukr. J. Phys. 2019. Vol. 64, No. 10 
69. M.J. Lighthill. Contributions to the theory of waves in nonlinear dispersive systems. IMA J. Appl. Math. 1, 269 (1965).

70. C. Mathieu, J. Jorzick, A. Frank, S.O. Demokritov, A.N. Slavin, B. Hillebrands, B. Bartenlian, C. Chappert, D. Decanini, F. Rousseaux, E. Cambril. Lateral quantization of spin waves in micron size magnetic wires. Phys. Rev. Lett. 81, 3968 (1998).

71. B.A. Kalinikos, N.G. Kovshikov, A.N. Slavin. Observation of spin wave solitons in ferromagnetic films. JETP Lett. 38, 413 (1983)

72. B.A. Kalinikos, N.G. Kovshikov, A.N. Slavin. Envelope solitons and modulational instability of dipole-exchange spin waves in yttrium-iron garnet films. J. Exp. Theor. Phys. 94, 159 (1988).

73. B.A. Kalinikos, N.G. Kovshikov, P.A. Kolodin, A.N. Slavin. Observation of dipole-exchange spin-wave solitons in tangentially magnetized ferromagnetic films. Solid State Commun. 74, 989 (1990).

74. P. De Gasperis, R. Marcelli, G. Miccoli. Magnetostatic soliton propagation at microwave frequency in magnetic garnet films. Phys. Rev. Lett. 59, 481 (1987).

75. M.A. Tsankov, M. Chen, C.E. Patton. Forward volume wave microwave envelope solitons in yttrium-iron garnet films - propagation, decay, and collision. J. Appl. Phys. 76, 4274 (1994)

76. M. Chen, M.A. Tsankov, J.M. Nash, C.E. Patton. Backward volume wave microwave envelope solitons in yttrium iron garnet films. Phys. Rev. B 49, 12773 (1994).

77. N.G. Kovshikov, B.A. Kalinikos, C.E. Patton, E.S. Wright, J.M. Nash. Formation, propagation, reflection, and collision of microwave envelope solitons in yttrium iron garnet films. Phys. Rev. B 5415210 (1996).

78. B.A. Kalinikos, N.G. Kovshikov, C.E. Patton. Decay free microwave magnetic envelope soliton pulse trains in yttrium iron garnet thin films. Phys. Rev. Lett. 78, 2827 (1997).

79. M. Chen, M.A. Tsankov, J.M. Nash, C.E. Patton. Microwave magnetic envelope dark solitons in Yttrium Iron Garnet thin films. Phys. Rev. Lett. 70, 1707 (1993).

80. B.A. Kalinikos, M.M. Scott, C.E. Patton. Self-generation of fundamental dark solitons in magnetic films. Phys. Rev. Lett. 84, 4697 (2000).

81. A.N. Slavin. Thresholds of envelope soliton formation in a weakly dissipative medium. Phys. Rev. Lett. 77, 4644 (1996).

82. A.V. Bagada, G.A. Melkov, A.A. Serga, A.N. Slavin. Parametric interaction of dipolar spin-wave solitons with localized electromagnetic pumping. Phys. Rev. Lett. 79, 2137 (1997).

83. P.A. Kolodin, P. Kabos, C.E. Patton, B.A. Kalinikos, N.G. Kovshikov, M.P. Kostylev. Amplification of microwave magnetic envelope solitons in thin yttrium iron garnet films by parallel pumping. Phys. Rev. Lett. 80, 1976 (1998).

84. A.L. Gordon, G.A. Melkov, A.A. Serga, V.S. Tiberkevich, A.V. Bagada, A.N. Slavin. Phase conjugation of linear signals and solitons of magnetostatic waves. JETP Lett. 67, 913 (1998).

85. G.A. Melkov, Y.V. Kobljanskyj, A.A. Serga, V.S. Tiberkevich, A.N. Slavin. Nonlinear amplification and compression of envelope solitons by localized nonstationary parametric pumping. J. Appl. Phys. 89, 6689 (2001).

86. G.A. Melkov, Y.V. Kobljanskyj, A.A. Serga, V.S. Tiberkevich, A.N. Slavin. Parametric interaction of dipolar spin-wave solitons with localized electromagnetic pumping. Phys. Status Solidi A 189, 1007 (2002).

87. A.A. Serga, M.P. Kostylev, B.A. Kalinikos, S.O. Demokritov, B. Hillebrands, H. Benner. Parametric generation of solitonlike spin-wave pulses in ring resonators based on ferromagnetic films. JETP Lett. 77, 300 (2003).

88. A.A. Serga, S.O. Demokritov, B. Hillebrands, A.N. Slavin. Formation of envelope solitons from parametrically amplified and conjugated spin-wave pulses. J. Appl. Phys 93, 8758 (2003).

89. B.A. Kalinikos, N.G. Kovshikov, M.P. Kostylev, H. Benner. Self-generation of spin-wave envelope soliton trains with different periods. JETP Lett. 76, 253 (2002).

90. A.B. Ustinov, B.A. Kalinikos, V.E. Demidov, S.O. Demokritov. Generation of dense spin-wave soliton trains in active ring resonators. JETP Lett. 80, 052405 (2009).

91. A.A. Serga, S.O. Demokritov, B. Hillebrands, A.N. Slavin. Self-generation of two-dimensional spin-wave bullets. Phys. Rev. Lett. 92117203 (2004).

92. S.O. Demokritov, A.A. Serga, V.E. Demidov, B. Hillebrands, M.P. Kostylev, B.A. Kalinikos. Experimental observation of symmetry-breaking nonlinear modes in an active ring. Nature 426, 159 (2003).

93. A.B. Ustinov, V.E. Demidov, A.V. Kondrashov, B.A. Kalinikos, S.O. Demokritov. Observation of the chaotic spinwave soliton trains in magnetic films. Phys. Rev. Lett. 106, 017201 (2011).

94. Z.H. Wang, A. Hagerstrom, J.Q. Anderson, W. Tong, M.Z. Wu, L.D. Carr, R. Eykholt, B.A. Kalinikos. Chaotic spin-wave solitons in magnetic film feedback rings. Phys. Rev. Lett. 107, 114102 (2011).

95. M.Z. Wu, B.A. Kalinikos, L.D. Carr, C.E. Patton. Observation of spin-wave soliton fractals in magnetic film active feedback rings. Phys. Rev. Lett. 96, 187202 (2006).

96. M.Z. Wu, C.E. Patton. Experimental observation of Fermi-Pasta-Ulam recurrence in a nonlinear feedback ring system. Phys. Rev. Lett. 98, 047202 (2007).

97. V.E. Demidov, U.F. Hansen, O. Dzyapko, N. Koulev, S.O. Demokritov, A.N. Slavin. Formation of longitudinal patterns and dimensionality crossover of nonlinear spin waves in ferromagnetic stripes. Phys. Rev. B 74, 092407 (2006).

98. A.A. Serga, M.P. Kostylev, B. Hillebrands. Formation of guided spin-wave bullets in ferrimagnetic film stripes. Phys. Rev. Lett. 101, 137204 (2008).

99. M. Bauer, C. Mathieu, S.O. Demokritov, B. Hillebrands, P.A. Kolodin, S. Sure, H. Dotsch, V. Grimalsky, Y. Rapoport, A.N. Slavin. Direct observation of two- 
dimensional self-focusing of spin waves in magnetic films. Phys. Rev. B 56, R8483 (1997).

100. M. Bauer, O. Buttner, S.O. Demokritov, B. Hillebrands, V. Grimalsky, Y. Rapoport, A.N. Slavin. Observation of spatiotemporal self-focusing of spin waves in magnetic films. Phys. Rev. Lett. 81, 3769 (1998).

101. O. Buttner, M. Bauer, S.O. Demokritov, B. Hillebrands, M.P. Kostylev, B.A. Kalinikos, A.N. Slavin. Collisions of spin wave envelope solitons and self-focused spin-wave packets in yttrium iron garnet films. Phys. Rev. Lett. 82, 4320 (1999).

102. A.N. Slavin, O. Buttner, M. Bauer, S.O. Demokritov, B. Hillebrands, M.P. Kostylev, B.A. Kalinikos, V.V. Grimalsky, Y. Rapoport. Collision properties of quasi-onedimensional spin-wave solitons and two-dimensional spinwave bullets. Chaos 13, 693 (2003).

103. M.P. Kostylev, A.A. Serga, B. Hillebrands. Radiation of caustic beams from a collapsing bullet. Phys. Rev. Lett. 106, 134101 (2011).

104. W.H. Rippard, M.R. Pufall, S. Kaka, S.E. Russek, T.J. Silva. Direct-current induced dynamics in $\mathrm{Cog}_{90} \mathrm{Fe}_{10} / \mathrm{Ni}_{80} \mathrm{Fe}_{20}$ point contacts. Phys. Rev. Lett. 92, 027201 (2004).

105. A. Slavin, V. Tiberkevich. Spin wave mode excited by spin-polarized current in a magnetic nanocontact is a standing self-localized wave bullet. Phys. Rev. Lett. 95, 237201 (2005).

106. S. Bonetti, V. Tiberkevich, G. Consolo, G. Finocchio, P. Muduli, F. Mancoff, A. Slavin, J. Akerman. Experimental evidence of self-localized and propagating spin-wave modes in obliquely magnetized currentdriven nanocontacts. Phys. Rev. Lett. 105, 217204 (2010).

107. S. Bonetti, V. Puliafito, G. Consolo, V.S. Tiberkevich, A.N. Slavin, J. Akerman. Power and linewidth of propagating and localized modes in nanocontact spin-torque oscillators. Phys. Rev. B 85, 174427 (2012).

108. D. Backes, F. Macia, S. Bonetti, R. Kukreja, H. Ohldag, A.D. Kent. Direct observation of a localized magnetic soliton in a spin-transfer nanocontact. Phys. Rev. Lett. 115, 127205 (2015).

109. J.C. Slonczewski. Current-driven excitation of magnetic multilayers. J. Magn. Magn. Mater. 159, L1 (1996).

110. L. Berger. Emission of spin waves by a magnetic multilayer traversed by a current. Phys. Rev. B 54, 9353 (1996).

111. J.C. Slonczewski. Excitation of spin waves by an electric current. J. Magn. Magn. Mater. 195, L261 (1999).

112. O.R. Sulymenko, O.V. Prokopenko, V.S. Tyberkevych, A.N. Slavin, A.A. Serga. Bullets and droplets: Twodimensional spin-wave solitons in modern magnonics (Review Article). Low Temp. Phys. 44, 602 (2018).

113. S.N. Bose. Plancks gesetz und lichtquantenhypothese. Z. Phys. 26, 178 (1924).

114. A. Einstein. Quantentheorie des einatomigen idealen gases. Sitz. Ber. Preuss. Akad. Wiss. 22, 261 (1924).
115. A. Einstein. Quantentheorie des einatomigen idealen gases, 2. Abhandlung. Sitz. Ber. Preuss. Akad. Wiss. 23, 3 (1925).

116. M. H. Anderson, J. R. Ensher, M.R. Matthews, C.E. Wieman, E.A. Cornell. Observation of Bose-Einstein condensation in a dilute atomic vapor. Science 269, 198 (1995).

117. H. Fröhlich. Bose condensation of strongly excited longitudinal electric modes. Phys. Lett. A 26, 402 (1968).

118. A.S. Borovik-Romanov, Y.M. Bunkov, V.V. Dmitriev, Y.M. Mukharskiı̌. Long-lived induction signal in superfluid ${ }^{3}$ He-B. JETP Lett. 40, 1033 (1984).

119. Yu.D. Kalafati, V.L. Safonov. Thermodynamic approach in the theory of paramagnetic resonance of magnons. J. Exp. Theor. Phys. 68, 1162 (1989).

120. Yu.D. Kalafati, V.L. Safonov. Theory of quasiequilibrium effects in a system of magnons excited by incoherent pumping. J. Exp. Theor. Phys. 73, 836 (1991).

121. G.A. Melkov, A.A. Serga. Nonlinear parametric excitation of spin waves. Front. Magn. Reduc. Dimens. Syst. 555 (1998).

122. S. Demokritov, B. Hillebrands, A.N. Slavin. Brillouin light scattering studies of confined spin waves: linear and nonlinear confinement. Phys. Rep. 348, 441 (2001).

123. S.O. Demokritov, V.E. Demidov, O. Dzyapko, G.A. Melkov, A.A. Serga, B. Hillebrands, A.N. Slavin. Bose-Einstein condensation of quasi-equilibrium magnons at room temperature under pumping. Nature 443, 430 (2006).

124. A.A. Serga, T. Schneider, B. Hillebrands, M.P. Kostylev, A.N. Slavin. Shaping of microwave pulses using phasesensitive spin-wave amplifier. Appl. Phys. Lett. 90, 022502 (2007).

125. T. Neumann, A.A. Serga, B. Hillebrands. Probing of a parametrically pumped magnon gas with a nonresonant packet of traveling spin waves. Appl. Phys. Lett. 93, 252501 (2008).

126. A.A. Serga, C.W. Sandweg, V.I. Vasyuchka, M.B. Jungfleisch, B. Hillebrands, A. Kreisel, P. Kopietz, M.P. Kostylev. Brillouin light scattering spectroscopy of parametrically excited dipole-exchange magnons. Phys. Rev. B 86, 134403 (2012).

127. V.E. Demidov, O. Dzyapko, S.O. Demokritov, G.A. Melkov, A.N. Slavin. Thermalization of a parametrically driven magnon gas leading to Bose-Einstein condensation. Phys. Rev. Lett. 99, 037205 (2007).

128. P. Clausen, D.A. Bozhko, V.I. Vasyuchka, B. Hillebrands, G.A. Melkov, A.A. Serga. Stimulated thermalization of a parametrically driven magnon gas as a prerequisite for Bose-Einstein magnon condensation. Phys. Rev. B 91, 220402 (2015).

129. O. Dzyapko, V.E. Demidov, S.O. Demokritov, G.A. Melkov, V.L. Safonov. Monochromatic microwave radiation from the system of strongly excited magnons. Appl. Phys. Lett. 92, 162510 (2008).

130. A.V. Chumak, G.A. Melkov, V.E. Demidov, O. Dzyapko, V.L. Safonov, S.O. Demokritov. Bose-Einstein condensa-

ISSN 2071-0194. Ukr. J. Phys. 2019. Vol. 64, No. 10 
tion of magnons under incoherent pumping. Phys. Rev. Lett. 102, 187205 (2009).

131. S.M. Rezende. Theory of coherence in Bose-Einstein condensation phenomena in a microwave-driven interacting magnon gas. Phys. Rev. B 79, 174411 (2009).

132. B.A. Malomed, O. Dzyapko, V.E. Demidov, S.O. Demokritov. Ginzburg-Landau model of Bose-Einstein condensation of magnons. Phys. Rev. B 81, 024418 (2010).

133. V.E. Demidov, O. Dzyapko, M. Buchmeier, T. Stockhoff, G. Schmitz, G.A. Melkov, S.O. Demokritov. Magnon kinetics and Bose-Einstein condensation studied in phase space. Phys. Rev. Lett. 101, 257201 (2008).

134. D.A. Bozhko, P. Clausen, A.V. Chumak, Yu.V. Kobljanskyj, B. Hillebrands, A.A. Serga. Formation of BoseEinstein magnon condensate via dipolar and exchange thermalization channels. Low Temp. Phys. 41, 801 (2015).

135. D.A. Bozhko, P. Clausen, G.A. Melkov, V.S. L'vov, A. Pomyalov, V.I. Vasyuchka, A.V. Chumak, B. Hillebrands, A.A. Serga. Bottleneck accumulation of hybrid magnetoelastic bosons. Phys. Rev. Lett. 118, 237201 (2017).

136. A.A. Serga, V.S. Tiberkevich, C.W. Sandweg, V.I. Vasyuchka, D.A. Bozhko, A.V. Chumak, T. Neumann, B. Obry, G.A. Melkov, A.N. Slavin, B. Hillebrands. BoseEinstein condensation in an ultra-hot gas of pumped magnons. Nat. Commun. 5, 3452 (2014).

137. W. Wettling, W.D. Wilber, P. Kabos, C.E. Patton. Light scattering from parallel-pump instabilities in yttrium iron garnet. Phys. Rev. Lett. 51, 1680 (1983).

138. D.V. Slobodianiuk, O.V. Prokopenko. Kinetics of strongly nonequilibrium magnon gas leading to Bose-Einstein condensation. J. Nano- Electron. Phys. 9, 03033 (2017).

139. A.J.E. Kreil, D.A. Bozhko, H.Yu. Musiienko-Shmarova, V.I. Vasyuchka, V.S. L'vov, A. Pomyalov, B. Hillebrands, A.A. Serga. From kinetic instability to Bose-Einstein condensation and magnon supercurrents. Phys. Rev. Lett. 121, 077203 (2018).

140. G.A. Melkov, S.V. Sholom. Kinetic instability of spin waves in thin ferrite films. J. Exp. Theor. Phys. 72, 341 (1991).

141. M. Schneider, T. Brächer, V. Lauer, P. Pirro, D.A. Bozhko, A.A. Serga, H.Yu. Musiienko-Shmarova, B. Heinz, Q. Wang, T. Meyer, F. Heussner, S. Keller, E.Th. Papaioannou, B. Lägel, T. Löber, V.S. Tiberkevich, A.N. Slavin, C. Dubs, B. Hillebrands, A.V. Chumak. Bose-Einstein condensation of quasi-particles by rapid cooling. arXiv:1612.07305 (2016).

142. O. Dzyapko, V.E. Demidov, M. Buchmeier, T. Stockhoff, G. Schmitz, G.A. Melkov, S.O. Demokritov. Excitation of two spatially separated Bose-Einstein condensates of magnons. Phys. Rev. B 80, 060401 (2009).

143. P. Nowik-Boltyk, O. Dzyapko, V.E. Demidov, N.G. Berloff, S.O. Demokritov. Spatially nonuniform ground state and quantized vortices in a two-component Bose-Einstein condensate of magnons. Sci. Rep. 2, 482 (2012).
144. D.A. Bozhko, A.A. Serga, P. Clausen, V.I. Vasyuchka, F. Heussner, G.A. Melkov, A. Pomyalov, V.S. L'vov, B. Hillebrands. Supercurrent in a room-temperature Bose-Einstein magnon condensate. Nat. Phys. 12, 1057 (2016).

145. D.A. Bozhko, A.J.E. Kreil, H.Yu. Musiienko-Shmarova, A.A. Serga, A. Pomyalov, V.S. L'vov, B. Hillebrands. Bogoliubov waves and distant transport of magnon condensate at room temperature. Nat. Commun. 10, 2460 (2019).

146. O. Dzyapko, I. Lisenkov, P. Nowik-Boltyk, V.E. Demidov, S.O. Demokritov, B. Koene, A. Kirilyuk, T. Rasing, V. Tiberkevich, A. Slavin. Magnon-magnon interactions in a room-temperature magnonic Bose-Einstein condensate. Phys. Rev. B 96, 064438 (2017).

147. V. Tiberkevich, I.V. Borisenko, P. Nowik-Boltyk, V.E. Demidov, A.B. Rinkevich, S.O. Demokritov, A.N. Slavin. Excitation of coherent second sound waves in a dense magnon gas. Sci. Rep. 9, 9063 (2019).

148. A. Rückriegel, P. Kopietz, D.A. Bozhko, A.A. Serga, B. Hillebrands. Magnetoelastic modes and lifetime of magnons in thin yttrium iron garnet films. Phys. Rev. B 89, 184413 (2014).

149. G.E.W. Bauer, E. Saitoh, B.J. van Wees. Spin caloritronics. Nat. Mater. 11, 391 (2012).

150. K.-i. Uchida, J. Xiao, H. Adachi, J. Ohe, S. Takahashi, J. Ieda, T. Ota, Y. Kajiwara, H. Umezawa, H. Kawai, G.E.W. Bauer, S. Maekawa, E. Saitoh. Spin Seebeck insulator. Nat. Mater. 9, 894 (2010).

151. C.W. Sandweg, Y. Kajiwara, A.V. Chumak, A.A. Serga, V.I. Vasyuchka, M.B. Jungfleisch, E. Saitoh, B. Hillebrands. Spin pumping by parametrically excited exchange magnons. Phys. Rev. Lett. 106, 216601 (2011).

152. C.M. Jaworski, J. Yang, S. Mack, D.D. Awschalom, R.C. Myers, J.P. Heremans. Spin-Seebeck effect: A phonon driven spin distribution. Phys. Rev. Lett. 106, 186601 (2011).

153. J. Xiao, G.E.W. Bauer, K.-i. Uchida, E. Saitoh, S. Maekawa. Theory of magnondriven spin Seebeck effect. Phys. Rev. B 81, 214418 (2010).

154. H. Adachi, K.-i. Uchida, E. Saitoh, S. Maekawa. Theory of the spin Seebeck effect. Rep. Prog. Phys. 76, 036501 (2013).

155. A.A. Serga, A.V. Chumak, B. Hillebrands. YIG magnonics. J. Phys. D: Appl. Phys. 43, 264002 (2010).

156. V. Cherepanov, I. Kolokolov, V. L'vov. The saga of YIG: spectra, thermodynamics, interaction and relaxation of magnons in a complex magnet. Phys. Rep. - Rev. Sec. Phys. Lett. 229, 81 (1993).

157. M.B. Jungfleisch, T. An, K. Ando, Y. Kajiwara, K.-i. Uchida, V.I. Vasyuchka, A.V. Chumak, A.A. Serga, E. Saitoh, B. Hillebrands. Heat-induced damping modification in yttrium iron garnet/platinum hetero-structures. Appl. Phys. Lett. 102, 062417 (2013). 
158. E. Padrón-Hernández, A. Azevedo, S.M. Rezende. Amplification of spin waves by thermal spin-transfer torque. Phys. Rev. Lett. 107, 197203 (2011).

159. H. Chang, P.A.P. Janantha, J. Ding, T. Liu, K. Cline, J.N. Gelfand, W. Li, M.C. Marconi, M. Wu. Role of damping in spin Seebeck effect in yttrium iron garnet thin films. Sci. Adv. 3, e1601614 (2017).

160. B. Obry, V.I. Vasyuchka, A.V. Chumak, A.A. Serga, B. Hillebrands. Spin-wave propagation and transformation in a thermal gradient. Appl. Phys. Lett. 101, 192406 (2012).

161. T. Langner, D.A. Bozhko, S.A. Bunyaev, G.N. Kakazei, A.V. Chumak, A.A. Serga, B. Hillebrands, V.I. Vasyuchka. Spin-wave propagation through a magnonic crystal in a thermal gradient. J. Phys. D: Appl. Phys. 51, 344002 (2018).

162. M. Vogel, A.V. Chumak, E.H. Waller, T. Langner, V.I. Vasyuchka, B. Hillebrands, G. von Freymann. Optically reconfigurable magnetic materials. Nat. Phys. 11, 487 (2015)

163. T. An, V.I. Vasyuchka, K.-i. Uchida, A.V. Chumak, K. Yamaguchi, K. Harii, J. Ohe, M.B. Jungfleisch, Y. Kajiwara, H. Adachi, B. Hillebrands, S. Maekawa, E. Saitoh. Unidirectional spin-wave heat conveyer. Nat. Mater. 12, 549 (2013).

164. K. Uchida, S. Takahashi, K. Harii, J. Ieda, W. Koshibae, K. Ando, S. Maekawa, E. Saitoh. Observation of the spin Seebeck effect. Nature 455, 778 (2008).

165. K. Uchida, H. Adachi, T. Kikkawa, A. Kirihara, M. Ishida, S. Yorozu, S. Maekawa, E. Saitoh. Thermoelectric generation based on spin Seebeck effects. Proc. IEEE 104, 1946 (2016).

166. M. Agrawal, V.I. Vasyuchka, A.A. Serga, A.D. Karenowska, G.A. Melkov, B. Hillebrands. Direct measurement of magnon temperature: New insight into magno-nphonon coupling in magnetic insulators. Phys. Rev. Lett. 111, 107204 (2013).

167. D. Meier, D. Reinhardt, M. van Straaten, C. Klewe, M. Althammer, M. Schreier, S.T.B. Goennenwein, A. Gupta, M. Schmid, C.H. Back, J.-M. Schmalhorst, T. Kuschel, G. Reiss. Longitudinal spin Seebeck effect contribution in transverse spin Seebeck effect experiments in Pt/YIG and Pt/NFO. Nat. Commun. 6, 8211 (2015).

168. M. Agrawal, V.I. Vasyuchka, A.A. Serga, A. Kirihara, P. Pirro, T. Langner, M.B. Jungfleisch, A.V. Chumak, E.Th. Papaioannou, B. Hillebrands. Role of bulk-magnon transport in the temporal evolution of the longitudinal spin-Seebeck effect. Phys. Rev. B 89, 224414 (2014).

169. N. Roschewsky, M. Schreier, A. Kamra, F. Schade, K. Ganzhorn, S. Meyer, H. Huebl, S. Geprägs, R. Gross, S.T.B. Goennenwein. Time resolved spin Seebeck effect experiments. Appl. Phys. Lett. 104, 202410 (2014).

170. M. Agrawal, A.A. Serga, V. Lauer, E.Th. Papaioannou, B. Hillebrands, V.I. Vasyuchka. Microwave-induced spin currents in ferromagnetic-insulator/normal-metal bilayer system. Appl. Phys. Lett. 105, 092404 (2014).
171. A. Kehlberger, U. Ritzmann, D. Hinzke, E.-J. Guo, J. Cramer, G. Jakob, M.C. Onbasli, D.H. Kim, C.A. Ross, M.B. Jungfleisch, B. Hillebrands, U. Nowak, M. Kläui. Length scale of the spin Seebeck effect. Phys. Rev. Lett. 115, 096602 (2015).

172. M. Schreier, F. Kramer, H. Huebl, S. Geprägs, R. Gross, S.T.B. Goennenwein, T. Noack, T. Langner, A.A. Serga, B. Hillebrands, V.I. Vasyuchka. Spin Seebeck effect at microwave frequencies. Phys. Rev. B 93, 224430 (2016).

173. J. Kimling, G.-M. Choi, J.T. Brangham, T. MatallaWagner, T. Huebner, T. Kuschel, F. Yang, D.G. Cahill. Picosecond spin Seebeck effect. Phys. Rev. Lett. 118, 057201 (2017).

174. T.S. Seifert, S. Jaiswal, J. Barker, S.T. Weber, I. Razdolski, J. Cramer, O. Gueckstock, S.F. Maehrlein, L. Nadvornik, S. Watanabe, C. Ciccarelli, A. Melnikov, G. Jakob, M. Münzenberg, S.T.B. Goennenwein, G. Woltersdorf, B. Rethfeld, P.W. Brouwer, M. Wolf, M. Kläui, T. Kampfrath. Femtosecond formation dynamics of the spin Seebeck effect revealed by terahertz spectroscopy. Nat. Commun. 9, 2899 (2018).

175. T. Hioki, R. Iguchi, Z. Qiu, D. Hou, K. Uchida, E. Saitoh. Time-resolved study of field-induced suppression of longitudinal spin Seebeck effect. Appl. Phys. Express 10, 73002 (2017).

176. T.B. Noack, H.Yu. Musiienko-Shmarova, T. Langner, F. Heussner, V. Lauer, B. Heinz, D.A. Bozhko, V.I. Vasyuchka, A. Pomyalov, V.S. L'vov, B. Hillebrands, A.A. Serga. Spin Seebeck effect and ballistic transport of quasi-acoustic magnons in room-temperature yttrium iron garnet films. J. Phys. D: Appl. Phys. 51, 234003 (2018).

177. L.J. Cornelissen, J. Liu, R.A. Duine, J. Ben Youssef, B.J. van Wees. Long-distance transport of magnon spin information in a magnetic insulator at room temperature. Nat. Phys. 11, 1022 (2015).

178. J. Shan, L.J. Cornelissen, J. Liu, J. Ben Youssef, L. Liang, B.J. van Wees. Criteria for accurate determination of the magnon relaxation length from the nonlocal spin Seebeck effect. Phys. Rev. B 96, 184427 (2017).

179. K. Ganzhorn, T. Wimmer, J. Cramer, R. Schlitz, S. Geprägs, G. Jakob, R. Gross, H. Huebl, M. Kläui, S.T.B. Goennenwein. Temperature dependence of the nonlocal spin Seebeck effect in YIG/Pt nanostructures. AIP Advances 7, 085102 (2017).

180. A.M. Pogorily, S.M. Ryabchenko, A.I. Tovstolytkin. Spintronics. Basic phenomena. Trends and development. Ukr. J. Phys. Reviews 6, 37 (2010).

181. M. Tsoi, A.G.M. Jansen, J. Bass, W.C. Chiang, M. Seck, V. Tsoi, P. Wyder. Excitation of a magnetic multilayer by an electric current. Phys. Rev. Lett. 80, 4281 (1998).

182. S.I. Kiselev, J.C. Sankey, I.N. Krivorotov, N.C. Emley, R.J. Schoelkopf, R.A. Buhrman, D.C. Ralph. Microwave oscillations of a nanomagnet driven by a spin-polarized current. Nature 425, 380 (2003).

ISSN 2071-0194. Ukr. J. Phys. 2019. Vol. 64, No. 10 
183. S. Tsunegi, H. Kubota, K. Yakushiji, M. Konoto, S. Tamaru, A. Fukushima, H. Arai, H. Imamura, E. Grimaldi, R. Lebrun, J. Grollier, V. Cros, S. Yuasa. High emission power and $\mathrm{Q}$ factor in spin torque vortex oscillator consisting of FeB free layer. Appl. Phys. Express 7, 063009 (2014).

184. V.E. Demidov, S. Urazhdin, R. Liu, B. Divinskiy, A. Telegin, S.O. Demokritov. Excitation of coherent propagating spin waves by pure spin currents. Nature Commun. 7 , 10446 (2016).

185. J. Torrejon, M. Riou, F. A. Araujo, S. Tsunegi, G. Khalsa, D. Querlioz, P. Bortolotti, V. Cros, K. Yakushiji, A. Fukushima, H. Kubota, S. Yuasa, M. D. Stiles, J. Grollier. Neuromorphic computing with nanoscale spintronic oscillators. Nature 547, 428 (2017).

186. G. Gerhart, E. Bankowski, G.A. Melkov, V.S. Tiberkevich, A.N. Slavin. Angular dependence of the microwavegeneration threshold in a nanoscale spin-torque oscillator. Phys. Rev. B 76, 024437 (2007).

187. G. Consolo, B. Azzerboni, G. Gerhart, G.A. Melkov, V. Tiberkevich, A.N. Slavin. Excitation of self-localized spin-wave bullets by spin-polarized current in in-plane magnetized magnetic nanocontacts: A micromagnetic study. Phys. Rev. B 76, 144410 (2007).

188. V.E. Demidov, S. Urazhdin, G. de Loubens, O. Klein, V. Cros, A. Anane, S.O. Demokritov. Magnetization oscillations and waves driven by pure spin currents. Phys. Rep. 673, 1 (2017).

189. L. Yang, R. Verba, V. Tiberkevich, T. Schneider, A. Smith, Z. Duan, B. Youngblood, K. Lenz, J. Lindner, A.N. Slavin, I.N. Krivorotov. Reduction of phase noise in nanowire spin orbit torque oscillators. Sci. Rep. 5, 16942 (2015).

190. A. Slavin, V. Tiberkevich, Nonlinear auto-oscillator theory of microwave generation by spin-polarized current. IEEE Trans. Magn. 45, 1875 (2009).

191. F. Sanches, V. Tiberkevich, K.Y. Guslienko, J. Sinha, M. Hayashi, O. Prokopenko, A.N. Slavin. Current-driven gyrotropic mode of a magnetic vortex as a nonisochronous auto-oscillator. Phys. Rev. B 89, 140410 (2014).

192. O. Prokopenko, E. Bankowski, T. Meitzler, V. Tiberkevich, A. Slavin. Spin-torque nano-oscillator as a microwave signal source. IEEE Magn. Lett. 2, 3000104 (2011).

193. O.R. Sulymenko, O.V. Prokopenko, V.S. Tiberkevich, A.N. Slavin, B.A. Ivanov, R.S. Khymyn. Terahertzfrequency spin Hall auto-oscillator based on a canted antiferromagnet. Phys. Rev. Appl. 8, 064007 (2017).

194. G.E. Rowlands, I.N. Krivorotov. Magnetization dynamics in a dual free-layer spin-torque nano-oscillator. Phys. Rev. $B$ 86, 094425 (2012).

195. T. Moriyama, G. Finocchio, M. Carpentieri, B. Azzerboni, D.C. Ralph, R.A. Buhrman. Phase locking and frequency doubling in spin-transfer-torque oscillators with two coupled free layers. Phys. Rev. B 86, 060411(R) (2012).
196. O.V. Prokopenko, I.N. Krivorotov, E.N. Bankowski, T.J. Meitzler, V.S. Tiberkevich, A.N. Slavin. Hysteresis regime in the operation of a dual-free-layer spin-torque nano-oscillator with out-of-plane counterprecessing magnetic moments. J. Appl. Phys. 114, 173904 (2013).

197. A.A. Tulapurkar, Y. Suzuki, A. Fukushima, H. Kubota, H. Maehara, K. Tsunekawa, D.D. Djayaprawira, N. Watanabe, S. Yuasa. Spin-torque diode effect in magnetic tunnel junctions. Nature 438, 339 (2005).

198. O.V. Prokopenko, I.N. Krivorotov, E. Bankowski, T. Meitzler, S. Jaroch, V.S. Tiberkevich, A.N. Slavin. Spin-torque microwave detector with out-of-plane precessing magnetic moment. J. Appl. Phys. 111, 123904 (2012).

199. O. Prokopenko, G. Melkov, E. Bankowski, T. Meitzler, V. Tiberkevich, A. Slavin, Noise properties of a resonancetype spin-torque microwave detector. Appl. Phys. Lett. 99, 032507 (2011).

200. O.V. Prokopenko, E. Bankowski, T. Meitzler, V.S. Tiberkevich, A.N. Slavin. Influence of temperature on the performance of a spin-torque microwave detector. IEEE Trans. Magn. 48, 3807 (2012).

201. O.V. Prokopenko, I.N. Krivorotov, T.J. Meitzler, E. Bankowski, V.S. Tiberkevich, A.N. Slavin. Spin-torque microwave detectors, In Magnonics. Topics in Applied Physics, vol 125, ed. by S. Demokritov, A. Slavin (Springer, Berlin, 2013), p. 143.

202. O.V. Prokopenko, A.N. Slavin. Microwave detectors based on the spin-torque diode effect. Low Temp. Phys. 41, 353 (2015).

203. S. Louis, O. Sulymenko, V. Tiberkevich, J. Li, D. Aloi, O. Prokopenko, I. Krivorotov, E. Bankowski, T. Meitzler, A. Slavin. Ultra-fast wide band spectrum analyzer based on a rapidly tuned spin-torque nano-oscillator. Appl. Phys. Lett. 113, 112401 (2018).

204. V. Baltz, A. Manchon, M. Tsoi, T. Moriyama, T. Ono, Y. Tserkovnyak. Antiferromagnetic spintronics. Rev. Mod. Phys. 90, 015005 (2018).

205. E.V. Gomonay, V.M. Loktev. Spintronics of antiferromagnetic systems. Low Temp. Phys. 40, 17 (2014).

206. R. Khymyn, I. Lisenkov, V. Tiberkevich, B.A. Ivanov, A. Slavin. Antiferromagnetic THz-frequency Josephsonlike oscillator driven by spin current. Sci. Rep. 7, 43705 (2017).

207. O.R. Sulymenko, O.V. Prokopenko, V.S. Tyberkevych, A.N. Slavin. Terahertz-frequency signal source based on an antiferromagnetic tunnel junction. IEEE Magn. Lett. 9, 3104605 (2018).

208. R. Khymyn, I. Lisenkov, J. Voorheis, O. Sulymenko, O. Prokopenko, V. Tiberkevich, J. Akerman, A. Slavin. Ultra-fast artificial neuron: generation of picosecondduration spikes in a current-driven antiferromagnetic auto-oscillator. Sci. Rep. 8, 15727 (2018). 
209. O. Sulymenko, O. Prokopenko, I. Lisenkov, J. Åkerman, V. Tyberkevych, A.N. Slavin, R. Khymyn. Ultra-fast logic devices using artificial "neurons" based on antiferromagnetic pulse generators. J. Appl. Phys. 124, 152115 (2018).

210. S. Miwa, M. Suzuki, M. Tsujikawa, T. Nozaki, T. Nakamura, M. Shirai, S. Yuasa, Y. Suzuki. Perpendicular magnetic anisotropy and its electric-field-induced change at metal-dielectric interfaces. J. Phys. D: Appl. Phys. 52, 063001 (2018).

211. T. Nozaki,T. Yamamoto, S. Miwa, M. Tsujikawa, M. Shirai, S. Yuasa, Y. Suzuki. Recent progress in the voltagecontrolled magnetic anisotropy effect and the challenges faced in developing voltage-torque MRAM. Micromachines 10, 327 (2019).

212. R. Verba, M. Carpentieri, G. Finocchio, V. Tiberkevich, A. Slavin. Parametric excitation and amplification of spin waves in ultrathin ferromagnetic nanowires by microwave electric field, In Spin wave confinement: Propagating waves (2nd edition), ed. by S.O. Demokritov (Pan Stanford Publishing Pte. Ltd., Singapore, 2017), p. 385.

213. Y.-J. Chen, H.K. Lee, R. Verba, J.A. Katine, I. Barsukov, V. Tiberkevich, J.Q. Xiao, A.N. Slavin, I.N. Krivorotov. Parametric resonance of magnetization excited by electric field. Nano Lett. 17, 572 (2017).

214. R. Verba, M. Carpentieri, Y.-J. Chen, I.N. Krivorotov, G. Finocchio, V. Tiberkevich, A. Slavin. Correction of phase errors in a spin-wave transmission line by nonadiabatic parametric pumping. Phys. Rev. Appl. 11, 054040 (2019).

215. G. Gubbiotti, S. Tacchi, M. Madami, G. Carlotti, A.O. Adeyeye, M. Kostylev. Brillouin light scattering studies of planar metallic magnonic crystals. J. Phys. D: Appl. Phys. 43, 264003 (2010).

216. B. Lenk, H. Ulrichs, F. Garbs, M. Münzenberg. The building blocks of magnonics. Phys. Rep. 507, 107 (2011).

217. M. Krawczyk, D. Grundler. Review and prospects of magnonic crystals and devices with reprogrammable band structure. J. Phys. Condens. Matter 26, 123202 (2014).

218. A.V. Chumak, V.I. Vasyuchka, A.A. Serga, B. Hillebrands. Magnon spintronics. Nat. Phys. 11, 453 (2015).

219. A.V. Chumak, A.D. Karenowska, A.A. Serga, B. Hillebrands. The dynamic magnonic crystal: New horizons in artificial crystal based signal processing. In: S.O. Demokritov, A.N. Slavin (eds.) Topics in Applied Physics, Vol. 125: Magnonics From Fundamentals to Applications (Springer, 2013), p. 243

220. A.V. Chumak, A.A. Serga, B. Hillebrands. Magnonic crystals for data processing. J. Phys. D: Appl. Phys. 50, 244001 (2017).

221. A.V. Chumak, A.A. Serga, B. Hillebrands, M.P. Kostylev. Scattering of backward spin waves in a one-dimensional magnonic crystal. Appl. Phys. Lett. 93, 022508 (2008).
222. A.V. Chumak, A.A. Serga, S. Wolff, B. Hillebrands, M.P. Kostylev. Design and optimization of onedimensional ferrite-film based magnonic crystal. J. Appl. Phys. 105, 083906 (2009).

223. A.V Chumak, A.A. Serga, S. Wolff, B. Hillebrands, M.P. Kostylev. Scattering of surface and volume spin waves in a magnonic crystal, Appl. Phys. Lett. 94, 172511 (2009).

224. A.D. Karenowska, A.V. Chumak, A.A. Serga, J.F. Gregg, B. Hillebrands. Magnonic crystal based forced dominant wavenumber selection in a spin-wave active ring. Appl. Phys. Lett. 96, 082505 (2010).

225. A.D. Karenowska, A.V. Chumak, A.A. Serga, J.F. Gregg, B. Hillebrands. Employing magnonic crystals to dictate the characteristics of auto-oscillatory spin-wave systems. J. Phys.: Conf. Ser. 303, 012007 (2011).

226. A.V. Chumak, V.I. Vasyuchka, A.A. Serga, M.P. Kostylev, V.S. Tiberkevich, B. Hillebrands. Storage-recovery phenomenon in magnonic crystal. Phys. Rev. Lett. 108, 257207 (2012).

227. A.V. Chumak, A.A. Serga, B. Hillebrands. Magnon transistors for all-magnon data processing. Nat. Commun. 5, 4700 (2014).

228. A.V. Chumak, P. Pirro, A.A. Serga, M.P. Kostylev, R.L. Stamps, H. Schultheiss, K. Vogt, S.J. Hermsdoerfer, B. Laegel, P.A. Beck, B. Hillebrands. Spin-wave propagation in a micro-structured magnonic crystal. Appl. Phys. Lett. 95, 262508 (2009).

229. F. Ciubotaru, A.V. Chumak, N.Yu. Grigoryeva, A.A. Serga, B. Hillebrands. Magnonic band gap design by the edge modulation of micro-sized waveguides. J. Phys. D: Appl. Phys. 45, 255002 (2012).

230. A.A. Nikitin, A.B. Ustinov, A.A. Semenov, A.V. Chumak, A.A. Serga, V.I. Vasuchka, E. Lähderanta, B.A. Kalinikos, B. Hillebrands. A spin-wave logic gate based on a width-modulated dynamic magnonic crystal. Appl. Phys. Lett. 106, 102405 (2015).

231. B. Obry, P. Pirro, T. Brächer, A.V. Chumak, J. Osten, F. Ciubotaru, A.A. Serga, J. Fassbender, B. Hillebrands. A micro-structured ion-implanted magnonic crystal. Appl. Phys. Lett. 102, 202403 (2013).

232. F. Ciubotaru, A.V. Chumak, B. Obry, A.A. Serga, B. Hillebrands. Magnonic band gaps in wave-guides with a periodic variation of the saturation magnetization. Phys. Rev. $B$ 88, 134406 (2013).

233. A.V. Chumak, T. Neumann, A.A. Serga, B. Hillebrands, M.P. Kostylev. A current-controlled, dynamic magnonic crystal. J. Phys. D: Appl. Phys. 42, 205005 (2009).

234. A.V. Chumak, V.S. Tiberkevich, A.D. Karenowska, A.A. Serga, J.F. Gregg, A.N. Slavin, B. Hillebrands. Alllinear time reversal by a dynamic artificial crystal. Nat. Commun. 1, 141 (2010).

235. A.D. Karenowska, J.F. Gregg, V.S. Tiberkevich, A.N. Slavin, A.V. Chumak, A.A. Serga, B. Hillebrands. Oscillatory energy exchange between waves coupled by a dy-

ISSN 2071-0194. Ukr. J. Phys. 2019. Vol. 64, No. 10 
namic artificial crystal. Phys. Rev. Lett. 108, 015505 (2012).

236. A.V. Chumak, P. Dhagat, A. Jander, A.A. Serga, B. Hillebrands. Reverse Doppler effect of magnons with negative group velocity scattered from a moving Bragg grating. Phys. Rev. B 81, 140404(R) (2010).

237. O.V. Dobrovolskiy, R. Sachser, T. Brächer, T. Böttcher, V.V. Kruglyak, R.V. Vovk, V.A. Shklovskij, M. Huth, B. Hillebrands, A.V. Chumak. Magnon-fluxon interaction in a ferromagnet/superconductor heterostructure. Nat. Phys. 15, 477 (2019).

238. T. Schneider, A.A. Serga, A.V. Chumak, B. Hillebrands, R.L. Stamps, M.P. Kostylev. Spin-wave tunneling through a mechanical gap. Europhys. Lett. 90, 27003 (2010).

239. M.P. Kostylev, A.A. Serga, T. Schneider, T. Neumann, B. Leven, B. Hillebrands, R.L. Stamps. Resonant and nonresonant scattering of dipole-dominated spin waves from a region of inhomogeneous magnetic field in a ferromagnetic film. Phys. Rev. B 76, 184419 (2007).

240. T. Neumann, A.A. Serga, B. Hillebrands, M.P. Kostylev. Frequency-dependent reflection of spin waves from a magnetic inhomogeneity induced by a surface direct current. Appl. Phys. Lett. 94, 042503 (2009).

241. A.A. Serga, T. Neumann, A.V. Chumak, B. Hillebrands. Generation of spin-wave pulse trains by current-controlled magnetic mirrors. Appl. Phys. Lett. 94, 112501 (2009).

242. M. Mohseni, R. Verba, T. Brächer, Q. Wang, D.A. Bozhko, B. Hillebrands, P. Pirro. Backscattering immunity of dipole-exchange magnetostatic surface spin waves. Phys. Rev. Lett. 122, 197201 (2019).

243. Q. Wang, A.V. Chumak, L. Jin, H. Zhang, B. Hillebrands, Z. Zhong. Voltage-controlled nanoscale reconfigurable magnonic crystal. Phys. Rev. B 95, 134433 (2017).

244. G.N. Kakazei, X.M. Liu, J. Ding, A.O. Adeyeye. $\mathrm{Ni}_{80} \mathrm{Fe}_{20}$ film with periodically modulated thickness as a reconfigurable one-dimensional magnonic crystal. Appl. Phys. Lett. 104, 042403 (2014).

245. R.G. Kryshtal, A.V. Medved. Nonreciprocity of spin waves in magnonic crystals created by surface acoustic waves in structures with yttrium iron garnet. J. Magn. Magn. Mater. 395, 180 (2015).

246. R.G. Kryshtal, A.V. Medved. Surface acoustic wave in yttrium iron garnet as tunable magnonic crystals for sensors and signal processing applications. Appl. Phys. Lett. 100, 192410 (2012).

247. D.D. Stancil, B.E. Henty, A.G. Cepni, J.P. Van't Hof. Observation of an inverse Doppler shift from lefthanded dipolar spin waves. Phys. Rev. B. 74, 060404(R) (2006).

248. B.M. Lebed', A.V. Nikiforov, S.V. Yakovlev, I.A. Yakovlev. Spin-wave scattering from the magnetic vortical lattice in the high-T superconductor-ferrite film structure. Sov. Phys. Solid State 34351 (1992).
249. N.I. Polzikova Resonant interaction of magnetostatic waves with a lattice of magnetic flux vortices in a superconductor. Tech. Phys. Lett. 19, 712 (1993).

250. T. Baba. Slow light in photonic crystals. Nat. Photon. 2, 465 (2008).

251. A.A. Serga, A.V. Chumak, A. André, G.A. Melkov, A.N. Slavin, S.O. Demokritov, B. Hillebrands. Parametrically stimulated recovery of microwave signal stored in standing spin-wave modes of a magnetic film. Phys. Rev. Lett. 99, 227202 (2007).

252. G.A. Melkov, Y.V. Koblyanskiy, R.A. Slipets, A.V. Talalaevskij, A.N. Slavin. Nonlinear interactions of spin waves with parametric pumping in permalloy metal films. Phys. Rev. B 79, 134411 (2009).

253. S. Schäfer, V. Kegel, A.A. Serga, B. Hillebrands, M.P. Kostylev. Variable damping and coherence in a highdensity magnon gas. Phys. Rev. B 83, 184407 (2011).

254. Y. Kobljanskyj, G. Melkov, K. Guslienko, V. Novosad, S.D. Bader, M. Kostylev, A. Slavin. Nano-structured magnetic metamaterial with enhanced nonlinear properties. Sci. Rep. 2, 478 (2012).

255. K.Yu. Guslienko, Yu. Kobljanskyj, G. Melkov, V. Novosad, S.D. Bader, M. Kostylev, A. Slavin. Nonlinear spin waves in two-dimensional arrays of magnetic nanodots Ultrafast Magnetism I Book Series: Springer Proceedings in Physics 159 (Springer, 2015), p. 206.

256. Y. Kobljanskyj, D. Slobodianiuk, G. Melkov, K. Guslienko, V. Novosad, S. Bader, M. Kostylev, A. Slavin. Nonlinear dynamic properties of two-dimensional arrays of magnetic nanodots. Handbook of Nanomagnetism: Applications and Tools (Pan Stanford Publishing, 2015), p. 97.

257. J.D. Adam. Analog signal processing with microwave magnetics. Proc. IEEE 76, 159 (1988).

258. I.V. Zavislyak, M.A. Popov. Microwave properties and applications of Yttrium Iron Garnet (YIG) films: Current state of art and perspectives, In B.D. Volkerts (ed.) Yttrium: Compounds, Production and Applications (Nova Science Publishers, Inc., 2009), p. 87.

259. Y. Khivintsev, M. Ranjbar, D. Gutierrez, H. Chiang, A. Kozhevnikov, Y. Filimonov, A. Khitun. Prime factorization using magnonic holographic devices. J. Appl. Phys. 120, 123901 (2016).

260. T. Brächer, P. Pirro. An analog magnon adder for allmagnonic neurons. J. Appl. Phys. 124, 152119 (2018).

261. G. Csaba, A. Papp, W. Porod. Spin-wave based realization of optical computing primitives. J. Appl. Phys. 115, 17C741 (2014).

262. R. Hertel, W. Wulfhekel, J. Kirschner. Domain-wall induced phase shifts in spin waves. Phys. Rev. Lett. 93, 257202 (2004).

263. M.P. Kostylev, A.A. Serga, T. Schneider, B. Leven, B. Hillebrands. Spin-wave logical gates. Appl. Phys. Lett. 87, 153501 (2005).

264. T. Schneider, A.A. Serga, B. Leven, B. Hillebrands, R.L. Stamps, M.P. Kostylev. Realization of spin-wave logic gates. Appl. Phys. Lett. 92, 022505 (2008). 
265. R.L. Stamps, S. Breitkreutz, J. Akerman, A.V. Chumak, Y. Otani, G.E.W. Bauer, J.-U. Thiele, M. Bowen, S.A. Majetich, M. Kläui. Magnon spintronics in the "The 2014 magnetism roadmap". J. Phys. D: Appl. Phys. 47, 333001 (2014).

266. K-S. Lee, S-K. Kim. Conceptual design of spin-wave logic gates based on a Mach-Zehnder-type spin-wave interferometer for universal logic functions. J. Appl. Phys. 104, 053909 (2008).

267. Q. Wang, R. Verba, T. Brächer, P. Pirro, A.V. Chumak. Integrated magnonic half-adder. arXiv:1902.02855 (2019).

268. A.V. Sadovnikov, E.N. Beginin, S.E. Sheshukova, D.V. Romanenko, Yu.P. Sharaevskii, S.A. Nikitov. Directional multimode coupler for planar magnonics: Sidecoupled magnetic stripes. Appl. Phys. Lett. 107, 202405 (2015).

269. Q. Wang, P. Pirro, R. Verba, A. Slavin, B. Hillebrands, A.V. Chumak. Reconfigurable nanoscale spin-wave directional coupler. Sci. Adv. 4, e1701517 (2018).

270. Q. Wang, M. Kewenig, M. Schneider, R. Verba, B. Heinz, M. Geilen, M. Mohseni, B. Lägel, F. Ciubotaru, C. Adelmann, C. Dubs, P. Pirro, T. Brächer, A.V. Chumak. Realization of a nanoscale magnonic directional coupler for all-magnon circuits. arXiv:1905.12353 (2019).

271. A. Khitun, M. Bao, K.L. Wang. Magnonic logic circuits. J. Phys. D: Appl. Phys. 43, 264005 (2010).

272. S. Klingler, P. Pirro, T. Brächer, B. Leven, B. Hillebrands, A.V. Chumak. Design of a spin-wave majority gate employing mode selection. Appl. Phys. Lett. 105, 152410 (2014).

273. S. Klingler, P. Pirro, T. Brächer, B. Leven, B. Hillebrands, A.V. Chumak. Spin-wave logic devices based on isotropic forward volume magnetostatic waves. Appl. Phys. Lett. 106, 212406 (2015).
274. T. Fischer, M. Kewenig, D.A. Bozhko, A.A. Serga, I.I. Syvorotka, F. Ciubotaru, C. Adelmann, B. Hillebrands, A.V. Chumak. Experimental prototype of a spinwave majority gate. Appl. Phys. Lett. 110, 152401 (2017).

275. Q. Wang, B. Heinz, R. Verba, M. Kewenig, P. Pirro, M. Schneider, T. Meyer, B. Lägel, C. Dubs, T. Brächer, A.V. Chumak. Spin pinning and spin-wave dispersion in nanoscopic ferromagnetic waveguides. Phys. Rev. Lett. 122, 247202 (2019).

276. C. Liu, J. Chen, T. Liu, F. Heimbach, H. Yu, Y. Xiao, J. Hu, M. Liu, H. Chang, T. Stueckler, S. Tu, Y. Zhang, Y. Zhang, P. Gao, Z. Liao, D. Yu, K. Xia, N. Lei, W. Zhao, M. Wu. Long-distance propagation of short-wavelength spin waves. Nat. Commun. 9, 738 (2018).

Received 26.08.19

О.В. Прокопенко, Д.А. Божко,

В.С. Тиберкевич, А.В. Чумак, В.І. Васючка, О.О. Серга,

О. Дзяпко, Р.В. Верба, О.В. Талалаєвсъкий,

Д.В. Слободянюк, Ю.В. Коблянсъкий,

В.А. Мойсеєнко, С.В. Шолом, В.Ю. Малишев

СУЧАСНІ ТЕНДЕНЦІЇ

РОЗВИТКУ МІКРОХВИЛЬОВОГО

МАГНЕТИЗМУ ТА НАДПРОВІДНОСТІ

$\mathrm{P}$ е $з$ ю м е

У статті розглядаються тенденції розвитку мікрохвильового магнетизму та надпровідності протягом останніх п'яти десятиліть. Огляд містить ключові результати останніх досліджень, що стосуються перспективних областей сучасного магнетизму та прикладної фізики - спінтроніки, магноніки, магнонної калоритроніки, фізики магнонних кристалів, спін-хвильової логіки тощо, а також розробки новітніх мікро- та нанорозмірних магнітних пристроїв. Основні досягнення в цих галузях фізики підсумовані та узагальнені. 